\title{
Selection for higher fertility reflects in the seminal fluid proteome of modern domestic chicken
}

Mohammad Atikuzzaman, Libia Sanz, Davinia Pla, Manuel Alvarez-Rodriguez, Marie Rubér, Dominic Wright, Juan J. Calvete and Heriberto Rodriguez-Martinez

\section{Journal Article}

\section{Tweet}

N.B.: When citing this work, cite the original article.

Original Publication:

Mohammad Atikuzzaman, Libia Sanz, Davinia Pla, Manuel Alvarez-Rodriguez, Marie Rubér, Dominic Wright, Juan J. Calvete and Heriberto Rodriguez-Martinez, Selection for higher fertility reflects in the seminal fluid proteome of modern domestic chicken, Comparative Biochemistry and Physiology - Part D, 2017. 21, pp.27-40.

http://dx.doi.org/10.1016/j.cbd.2016.10.006

Copyright: Elsevier

http://www.elsevier.com/

Postprint available at: Linköping University Electronic Press

http://urn.kb.se/resolve?urn=urn:nbn:se:liu:diva-132624
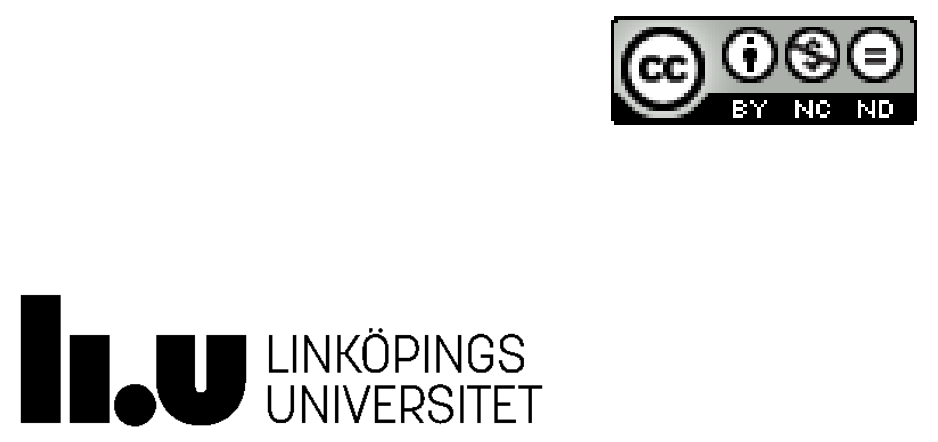
Comparative Biochemistry and Physiology, part D (MS. 26313, accepted for publication)

\section{Selection for higher fertility reflects in the seminal fluid proteome of modern domestic chicken}

Mohammad ATIKUZZAMAN ${ }^{1, *}$, Libia SANZ ${ }^{2, *}$, Davinia PLA², Manuel ALVAREZ-

RODRIGUEZ ${ }^{1}$, Marie RUBÉR ${ }^{1}$, Dominic WRIGHT ${ }^{3}$, Juan J. CALVETE ${ }^{2, \ddagger}$, Heriberto RODRIGUEZ-MARTINEZ ${ }^{1, \ddagger}$

${ }^{1}$ Department of Clinical and Experimental Medicine, ${ }^{3}$ Department of Physics, Chemistry and Biology, University of Linköping, Linköping, Sweden

${ }^{2}$ Instituto de Biomedicina de Valencia, CSIC, Valencia, Spain

* These authors have made equal contribution to the work, and should both be considered "first authors".

Running title: Comparative seminal fluid proteomics of high and low egg-laying chicken

${ }^{\ddagger}$ Corresponding authors: For questions concerning biological aspects of this work, please contact Heriberto Rodriguez-Martinez (heriberto.rodriguez-martinez@liu.se), Linköping University, Department of Clinical and Experimental Medicine, Faculty of Medicine and Health Sciences; Lasarettsgatan 64/65, Lanken, Floor 12, SE-581 85 Linköping, Sweden; For issues regarding proteomic analysis, please contact Juan J. Calvete (jcalvete@ibv.csic.es), Laboratorio de Venómica Estructural y Funcional, Instituto de Biomedicina de Valencia, C.S.I.C., Jaime Roig 11, 46010 Valencia, Spain. Phone: +34 96339 1778, Fax: 34963690800

\begin{tabular}{ll}
\multicolumn{2}{l}{ Abbreviations } \\
WL & White Leghorn \\
RJF & Red Junglefowl \\
AIL & Advanced intercross line \\
SF & seminal fluid \\
TGF- $\beta 2$ & transforming growth factor-beta 2 \\
CXCL10 & C-X-C motif chemokine 10
\end{tabular}

Key words: Rooster seminal fluid proteome; cytokines; egg-laying capacity; Red Junglefowl; White Leghorn; Advanced Intercross Line; chicken 


\begin{abstract}
The high egg-laying capacity of the modern domestic chicken (i.e. White Leghorn, WL) has arisen from the low egg-laying ancestor Red Junglefowl (RJF) via continuous trait selection and breeding. To investigate whether this long-term selection impacted the seminal fluid (SF)proteome, 2DE electrophoresis-based proteomic analyses and immunoassays were conducted to map SF-proteins/cytokines in RJF, WL and a $9^{\text {th }}$ generation Advanced Intercross Line (AIL) of RJF/WL-L13, including individual SF ( $n=4$, from each RJF, WL and AIL groups) and pools of the SF from 15 males of each group, analyzed by 2DE to determine their degree of intra-group (AIL, WL, and RJF) variability using Principal Component Analysis (PCA); respectively an inter-breed comparative analysis of intergroup fold change of specific SF protein spots intensity between breeds. The PCA clearly highlighted a clear intra-group similarity among individual roosters as well as a clear inter-group variability (e.g. between RJF, WL and AIL) validating the use of pools to minimize confounding individual variation. Protein expression varied considerably for processes related to sperm motility, nutrition, transport and survival in the female, including signaling towards immunomodulation. The major conserved SF-proteins were serum albumin and ovotransferrin. Aspartate aminotransferase, annexin A5, arginosuccinate synthase, glutathione S-transferase 2 and L-lactate dehydrogenase-A were RJF-specific. Glyceraldehyde-3-phosphate dehydrogenase appeared specific to the WL-SF while angiotensinconverting enzyme, $\gamma$-enolase, coagulation factor IX, fibrinogen $\alpha$-chain, hemoglobin subunit $\alpha$ D, lysozyme C, phosphoglycerate kinase, Src-substrate protein p85, tubulins and thioredoxin were AIL-specific. The RJF-SF contained fewer immune system process proteins and lower amounts of the anti-inflammatory/immunomodulatory TGF- $\beta 2$ compared to WL and AIL, which had low levels- or lacked pro-inflammatory CXCL10 compared to RJF. The seminal fluid proteome differs between ancestor and modern chicken, with a clear enrichment of proteins and peptides related to immune-modulation for sperm survival in the female and fertility.
\end{abstract}

\title{
Highlights
}

- Seminal fluid proteomes of high- (WL) and low (RJF) egg-laying-capacity roosters were analyzed

- Seminal fluid proteome of 9th generation RJF x WL-L13 interbreed line was analyzed

- Cytokine/chemokine profiles of WL, RJF, and RJF x WL-L13 seminal fluids were determined

- Considerable variation in expression of seminal fluid proteins/cytokines was observed

- Selection for higher fertility has an impact in the composition of the seminal fluid proteome

-Ancestor and modern chicken seminal fluids varied in immune modulatory proteins/cytokines which might impact production performance 


\section{Introduction}

Semen is composed of a complex mixture of organic and inorganic components built by secretions of the male reproductive organs, which the ejaculated spermatozoa bathe in (Mann et al., 1982; Blesbois and Hermier, 1990). In mammals, seminal plasma contains a huge bulk of proteins and peptides (25-60 g/L) per ejaculate, mostly derived from the accessory sex glands (Batruch et al., 2011; Rodríguez-Martínez et al., 2011). In the chicken, due to a lack of accessory sex glands, with the exception of the vascular bodies in the cloaca which resemble the mammalian bulbo-urethral glands, the seminal fluid derives from the testis, the rudimentary epididymis and the ductus deferens (Etches, 1996; Fujihara, 1992) and contains a 10-fold lower protein load than mammals (2.0-2.4 g/dL) (Harris and Sweeney, 1971; Thurston et al., 1982). In mammals, seminal plasma proteins have been ascribed a range of diverse functions, including germicidal effects, promotion of sperm survival, assistance in sperm interactions with different microenvironments in the female genital tract, and the signaling to the immune system of the female so that a state of maternal tolerance to foreign spermatozoa (and embryos and placentae) is established (Rodríguez-Martínez et al., 1998, 2008, 2011; Novak et al., 2010; Kareskoski et al., 2011; Caballero et al., 2012; Druart et al., 2013; Milardi et al., 2013; Rodrigues et al., 2013; Sharma et al., 2013; Bromfield et al., 2014). Whether such roles can also be ascribed to avian seminal fluid (SF) proteins remains to be explored.

Proteomic research in Reproductive Biology, accelerated by methodological developments in mass spectrometry (Druart et al., 2013; Rodrigues et al., 2013; Sharma et al., 2013; Calvete et al., 1994; Pilch and Mann, 2006; Kelly et al., 2006), has increased our knowledge of the composition of seminal plasma in mammals, revealing relevant roles for sperm function (Soggiu et al., 2013; Soler et al., 2016) and immune modulation, including the role of specific cytokines and chemokines (Schjenken and Robertson, 2014; Crawford et al., 2015) with relevance for fertility. Proteomic studies investigating avian sperm and ejaculates are comparatively scarce, yet those that do exist assess the potential correlations with sperm motility (Froman et al., 2011), attempt to identify and classify proteins (Marzoni et al., 2013), or correlate the proteome to the semen phenotype (Labas et al., 2015). Few studies (Marzoni et al., 2013; Labas et al., 2015) describe seminal plasma proteins (e.g. beta-defensin as gallinacins, ovotransferrin, serum albumin and peroxiredoxin-6) and their role in antimicrobial activity and sperm survival (Das et al., 2011). Comparative cytokine/chemokine studies are also restricted to cytokine expression in avian testis (Ocón-Grove et al., 2010; Michailidis et al., 2014) and the oviduct. In the latter, proinflammatory cytokines (tumor necrosis factor (TNF) and interleukin (IL)-1 $\beta$ ) as well as immunosuppressive cytokines (as transforming growth factor (TGF)- $\beta$ ) and their receptors are expressed in the vagina and utero-vaginal-junction respectively, after seminal deposition (Das et al., 2006, 2008, 2009). The lack of cytokine mappings in the chicken SF, which might be an important factor for sperm function, comparative to what is known in mammals (Barranco et al., 2015), is thus required.

Fertility varies among animals, irrespective of classes. Red Junglefowl (RJF), the wild progenitor of modern domestic chicken breeds, has a low fertility, expressed in terms of fertile oviposition rates per season, while domestic modern laying chicken breeds such as the White Leghorn (WL) 
display the opposite situation, with high laying/fertility rates. Sperm quality and fertility varies among males, particularly when considering the pressure of selection applied. Interestingly, when a chicken breed is selected for a particular trait- as egg production, a decrease in semen quality has been recorded in the male line (Murugesan et al., 2013). Comparative studies have shown differences in sperm concentration and forward sperm motility between wild RJF and domestic chicken with an advantage for the RJF, without consideration of eventual roles for the seminal fluid (Malik et al., 2013). The composition of the seminal fluid/plasma also varies with varying fertility among males, even if definite links with reproductive outcome are yet to be fully established. Of even greater relevance, semen deposition elicits gene expression shifts in the female genital tract (Fazeli et al., 2004; Atikuzzaman et al., 2015), calling for the identification of the pertinent signals involved, including components of the SF-proteome that could be related to sperm survival and immunomodulation by peptides as cytokines, an as yet undiscovered chapter in the rooster SF.

The present study aims to map the proteome, including cytokines, of the seminal fluid of the ancestor RJF ( $n=31$ birds), of the highly-selected modern WL ( $n=20$ birds), and of an AIL ( $n=$ 23 birds) - intercross between wild and domestic chicken (RJF x WL-L13), with clear differences in egg-laying/fertility, evolved during selection. The SF was analyzed for protein profiles as ejaculate pools per breed (15 males/breed) to minimize individual variation or as individual ejaculates (39-79 ejaculates/breed) or pool of ejaculates of a male (10 males/breed) for quantitation of cytokines.

\section{Material and methods}

\subsection{Animals and seminal fluid source}

Sexually mature, proven fertile roosters from a RJF-pure line, a pure WL line and from a $9^{\text {th }}$ generation AIL- intercross between RJF and WL-L13 lines have been used (see Johnsson et al., 2012 for details of the cross and breeds used as well as for details on rearing and breeding routines). Food and water were available ad libitum and the chicken were held under controlled temperature and light regimes (12h light: 12h darkness cycle, 5 lux) in 1-2 $\mathrm{m}^{2}$ pens depending on age for their first seven weeks, in compliance with European Community (Directive 2010/63/EU) and Swedish (SJVFS 2012:26) current legislation. Throughout all experiments, animals were handled carefully to avoid any unnecessary stress. The experiments were approved by the 'Regional Committee for Ethical Approval of Animal Experiments' (Linköpings Djurförsöksetiska nämnd) in Linköping, Sweden (permit no. 75-12).

Semen was collected from pre-trained roosters by manual abdominal massage. The success of ejaculation was confirmed by extending $2 \mu \mathrm{l}$ of semen in Dulbecco's medium (1:10 v/v) for examination on a Carl Zeiss microscope equipped with a thermal plate $\left(41^{\circ} \mathrm{C}\right)$ and positive phase contrast optics (10x objective). Ejaculates mixed with faeces or blood or lacking live sperm were excluded from the study. Semen from 31 RJF (45 ejaculates), 23 RJF/WL-L13 (43 ejaculates) and $20 \mathrm{WL}$ (79 ejaculates) roosters were finally selected for the study. The selected ejaculates were centrifuged at $+5^{\circ} \mathrm{C}, 21,000 \mathrm{xg}$ for 10 minutes immediately after collection to harvest the 
extra-cellular SF-supernatant, which was thereafter transferred to liquid nitrogen ( $\left.\mathrm{LN}_{2}\right)$ before storage at $-80^{0} \mathrm{C}$ prior to analysis.

\subsection{Identification of proteins by 2DE followed by mass spectrometry}

Seminal fluid samples collected from four animals per group (breed) were individually analyzed by 2DE to determine their degree of individual variability. As well, pools of SF from 15 males of each group (breed) were built a group/breed sample. The rationale behind this pooling was our interest in unveiling SF proteome differences that may underline a breed population fitness trait, rather than individual differences, and thus to minimize confounding individual intra- and intermale variation. Each breed pool was analyzed in triplicate by 2DE and only spots consistently found in all three experiments were considered in the subsequent inter-breed comparative analysis.

Sample preparation: $150 \mu$ g of SF from each breed pool (RJF, WL and AIL) was subjected to cleansing using a 2D clean-Up kit (GE Healthcare). The cleansed SF of each breed was suspended in $90 \mu \mathrm{l}$ of rehydration solution containing $7 \mathrm{M}$ urea, $2 \mathrm{M}$ thiourea, $4 \%(\mathrm{w} / \mathrm{v})$ CHAPS, $0.5 \%$ (v/v) IPG buffer, $40 \mathrm{mM}$ DTT plus $1 \mu \mathrm{l}$ of bromophenol blue. The solution, after centrifugation at $13,000 \mathrm{xg}$ for 5 minutes, was loaded into a prior overnight rehydrated (rehydration solution containing $7 \mathrm{M}$ urea, $2 \mathrm{M}$ thiourea, 2\% w/v CHAPS, 0.5\% v/v IPG buffer, 0.002\% bromophenol blue and $40 \mathrm{mM}$ DTT) seven cm long IPG strip (pH range 3-10 L).

2DE (two-dimensional gel electrophoresis): The isoelectric focusing was carried out following Calvete et al. (2009) at $20^{\circ} \mathrm{C}$ and $50 \mu \mathrm{A}$ using Ettan IPGphor 3 (GE Healthcare) with a modification of running schedule: First step and hold, $500 \mathrm{~V}$ (125 Vh); Second gradient, $1000 \mathrm{~V}$ (500 Vh); and Third gradient, $5000 \mathrm{~V}$ (6666 Vh). Prior to perform second dimension sodium dodecyl sulphate-polyacrylamide gel electrophoresis (SDS-PAGE), the IPG strip was equilibrated for 7 minutes twice in SDS equilibration buffer solution containing $6 \mathrm{M}$ urea, 75 $\mathrm{mM}$ Tris-HCl pH 8.8, 29.3\% glycerol (v/v), 2\% SDS (w/v) and 0.002\% bromophenol blue (w/v) with a gentle shaking at 15 rotations per min.

The IPG strips were then placed on a SDS polyacrylamide $4 \%$ stacking gel $(0.75 \mathrm{~mm}$ thick) with a SDS polyacrylamide 15\% separation gel (1.5 mm thick), according to Laemmli (1970), into a vertical SDS-PAGE gel slab filled with electrode buffer. A $200 \mathrm{kDa}$ molecular weight marker (Mark12 ${ }^{\mathrm{TM}}$, Invitrogen Corporation) was loaded on to the stacking gel before running the electrophoresis. The electrophoresis was performed at $100 \mathrm{~V}$ until the dye reached to the bottom of the separating gels (Calvete at al., 2009).

Protein spot visualization: 2DE gels of individual and pooled SF samples were stained with Coomassie Brilliant Blue G-colloidal at room temperature on a plate shaker at 15 rpm until the spots were visible. The stained gels were then washed with deionized water until the backgrounds became clear. Gels were stored for further analysis immediate after scanning with a high resolution scanner, LabScan (Amersham Pharmacia Biotech, Sweden). The scanned 2D-gel images were analyzed for intragroup (AIL, WL, and RJF) variability using MarkerView ${ }^{\mathrm{TM}}$ 1.2.1 
Software (AB Sciex, Ontario, Ca), and for intergroup fold change of specific protein spots intensity comparing AIL and WL with RJF, using SameSpots (TotalLab Ltd. Newcastle, UK).

Protein digestion and mass spectrometry: Immediately after scanning, the visible protein spots on 2D gels were cut and subjected to automated reduction with DTT and alkylation with iodoacetamide and digestion with sequencing grade modified porcine trypsin (Promega) using a ProGest $^{\mathrm{TM}}$ digestor (Genomic Solutions) following the manufacturer guidelines. The dried digests were re-suspended with $8 \mu \mathrm{l}$ of $0.1 \%$ formic acid, and $6 \mu \mathrm{l}$ aliquots injected and analyzed by Liquid Chromatography-Electrospray Ionization-Quadropole-Time-of-Flight-Mass Spectrometry (LC-ESI-Q-TOF-MS/MS) using a Synapt G2 instrument (Waters, Manchester, UK) hyphenated to a liquid nano-chromatographic separation system (NanoACQUITY-UPLC, Waters) equipped with a C18 column $(100 \mu \mathrm{m}$ x $100 \mathrm{~mm}, 1.7 \mu \mathrm{m}$ particles (BEH130 C18, Waters) operated at a flow rate of $0.6 \mu \mathrm{l} / \mathrm{min}$. The mobile phase consisted of $0.1 \%$ formic acid in water (A) and acetonitrile (B). The optimized ultra-performance liquid chromatography (UPLC) elution conditions were $0-1$ min $1-12 \% \mathrm{~B}$; $1-16 \mathrm{~min}, 12-40 \% \mathrm{~B} ; 16-18 \mathrm{~min}, 40-85 \% \mathrm{~B}$; $18-20$ $\min , 85-1 \% \mathrm{~B} ; 20-30 \mathrm{~min}, 1 \% \mathrm{~B}$. The autosampler was maintained at $+10{ }^{0} \mathrm{C}$. All the analyses were performed in data dependent analysis (DDA) mode that automatically triggers the MS/MS experiments. The scan range was from 100 to 2,000 m/z. For positive nanospray mode, the capillary and cone voltage were set at $3 \mathrm{kV}$ and $28 \mathrm{~V}$ respectively. The deviation gas was set at $100 \mathrm{~L} / \mathrm{h}$, the cone gas at $10 \mathrm{~L} / \mathrm{h}$ and the source temperature at $+100^{0} \mathrm{C}$. The mass spectrometer was operated in V-optics mode with 20,000 resolution using dynamic range extension. The data acquisition rate was set to one scan time. All analyses were acquired using the LockSpray to ensure accuracy and reproducibility. Leucine-enkephalin was used as the lockmass at a concentration of $200 \mathrm{ng} / \mathrm{ml}$ and a flow rate of $0.5 \mu \mathrm{l} / \mathrm{min}$. Data were collected in continuum mode and the spray frequency was set at $30 \mathrm{~s}$. The data acquisition and spectra were analyzed by MassLynx4.1 (Waters).

The Gallus gallus SF-proteins were identified from their peptide sequences using MASCOT MS/MS Ions Search Engine version 2.5 (Matrix Science, Boston, MA) along with the latest updated version of Swiss-Prot protein database (UniProtKB/Swiss-Prot). Carbamidomethyl cysteine and oxidized methionine residues were selected as fixed and variable modifications, respectively. The peptide tolerance and the MS/MS tolerance were set at $1.2 \mathrm{Da}$ and $0.6 \mathrm{Da}$, respectively and peptide charges were set at $2^{+}, 3^{+}$and $4^{+}$. Trypsin digestion (allowing two possible missed cleavages) and taxonomy of bony vertebrate were set. The data format and instrument information was set as Micromass (PKL) and ESI-QUAD-TOF, respectively along with decoy. The ion scores have been reported as $-10 x \log 10(\mathrm{P})$ where $\mathrm{P}$ is the probability that the observed match is a random even. Individual MS/MS peptide ion scores >39 indicated identity or extensive homology $(\mathrm{P}<0.05)$ for the MS/MS ion search have been taken into consideration for protein identification. Identified proteins underwent bioinformatic analysis using the PANTHER classification system (Version 10.0) (Mi et al., 2016).

\subsection{Measurement of the concentrations of cytokines and chemokines}

Presence and relative concentration of a battery of cytokines and chemokines including IL-6, CXCL8 (IL-8), CCL2 (monocyte chemo-attractant protein-1, MCP-1) or the growth factor 
granulocyte-macrophage colony-stimulating factor (GM-CSF, associated with a proinflammatory immune response); the anti-inflammatory IL-10 and TGF- $\beta 1$, TGF- $\beta 2$ and TGF$\beta 3$; the Th1-associated IFN- $\gamma$ and CXCL10 (interferon gamma-induced protein, IP-10); the Th2associated CCL22 (macrophage-derived chemokine, MDC); and the Th17-associated IL-17 and CXCL1 (growth-regulated oncogene, GRO), as well as the inducer of NK cell proliferation IL15 were first examined in the SF of individual ejaculates of RJF, WL and AIL roosters (n>15 birds per breed, range: 15-79 ejaculates) using a multiplexed microsphere-based flow cytometric assay (Luminex's xMAP ${ }^{\circledR}$ ) following the protocol of Barranco et al. (2015). In brief, pre-coated magnetic beads (Cat\#HCYTOMAG-60K-11 for human reactivity, Merck Millipore, Billerica, MA, USA) were used for the determination of cytokines and chemokines except TGF- $\beta$ s, while a 3-plex kit (Cat\#TGFB-64K-03 for pig, human, mouse, rat, hon-human primate, canine, feline reactivity, Merck Millipore) were used for TGF- $\beta$ s following the methods described by manufacturers in 96-well multiscreen plates, albeit samples were ran as singlets. A $25 \mu \mathrm{SF}$ (acidified in case of TGF- $\beta \mathrm{s}$ ) was used per sample well to measure the concentration.

Concentrations of TGF- $\beta 2$, TGF- $\beta 3$ and CXCL10 were further measured in pooled SF of each male (10 birds per breed) using a chicken-specific ELISA kit for TGF- $\beta$ s (Nori ${ }^{\mathrm{TM}}$ Chicken TGF$\beta 2$ and TGF- $\beta 3$ kit, Genorise Scientific, Inc., Glen Mills, PA, USA) and for CXCL10 (CXCL10 ELISA kit, Cat\#MBS2505839, MyBiosource, Inc., San Diego, CA, USA), after preparation of a standard curve, following the manufacturer protocol. The 96-well microplate loaded with duplicate-samples was incubated, washed using an automatic microplate washer (Ref\# 30022011, Hydroflex, Tecan Austria GmbH, Grödig, Austria) and the optical density of each well was determined using a microplate reader (Ref\# 16039400, Sunrise, Tecan Austria GmbH) at $450 \mathrm{~nm}$.

An independent t-test analysis was employed to compare the mean concentration values (expressed as $\mathrm{pg} / \mathrm{ml}$, mean \pm SEM) of cytokines and chemokines in the SF of the different breeds. $\mathrm{P}<0.05$ was considered significant.

\section{Results}

A Principal Component Analysis (PCA) of the 2DE images of 12 individual SF samples (4 per group RJF, WL and AIL) (Fig. 1) was performed in order to visualize the 2DE data in terms of sample grouping and gain insights into their intra-group and inter-group variability. Figure 1 (panel D) highlighted a clear intra-group similarity among individual rosters as well as a clear inter-group variability (e.g. between RJF, WL and AIL). Under such circumstances group/breed pools built by equal SF-amounts of 15 roosters per group were subjected to 2DE separation and compared using SameSpots, to analyze inter-group fold change of specific SF protein spots intensity between breeds.

\subsection{The major conserved SF-proteins were serum albumin and ovotransferrin}

Out of 107 (RJF), 52 (WL) and 98 (AIL) spots on the 2D gels analyzed (Fig. 2), a total of 28 (26.16\%), 21 (40.38\%) and 38 (38.78\%) proteins were detected in SF (Table 1 and Supplementary Table S1). Around 50\% of the detected SF-proteins (from the total number of 
spots analyzed per breed) were serum albumin (11.21\%, 21.15\%, and 13.27\%) and ovotransferrin (15.89\%, 17.31\%, and 12.24\%) in RJF, WL, and AIL, respectively (Table 1). Among the identified proteins, aspartate aminotransferase, annexin A5, arginosuccinate synthase, glutathione S-transferase 2 and L-lactate dehydrogenase-A were RJF-specific, while glyceraldehyde-3-phosphate dehydrogenase were found to be specific to the WL-SF. On the other hand, angiostensin-converting enzyme, $\gamma$-enolase, coagulation factor IX, fibrinogen $\alpha$ chain, hemoglobin subunit $\alpha-D$, lysozyme C, phosphoglycerate kinase, Src substrate protein p85, tubulins and thioredoxin were AIL specific (Table 1).

3.2 Eleven SF-proteins were down-expressed while eight proteins were over-expressed in RJF compared with WL and AIL

Compared to the WL, the down-regulated proteins (shown subsequently as fold changes, and spot number on the 2D gels) in the SF of the RJF were serum albumin $(4.3,126)$, ovotransferrin $(2.1,123)$, ovoinhibitor $(1.9,129)$, creatinine kinase B-type $(2.6,131), \alpha$-enolase $(5.0,132)$, Llactate dehydrogenase B chain $(5.3,140)$, triosephosphate isomerase $(9.1,145)$, retinol-binding protein $(3.4,146)$ and apolipoprotein A-l $(3.8,147)$ and over-expressed proteins in the SF of RJF were malate dehydrogenase $(1.7,139)$, glyceraldehyde-3-phosphate dehydrogenase $(3.0,141)$, cystatin $(2.7,152)$ and gallinacin-9 $(1.9,157)$ (Fig. 3A-B and Supplementary Table S2A). A similar comparison revealed down-regulated proteins in RJF, including serum albumin (3.2, 214), ovotransferrin $(4.0,192)$, phosphoglycerate mutase 1 (2.1, 230) and tubulin $ß-1$ chain $(2.0$, $172)$ while, the over-expressed proteins were transthyretin $(2.6,85), \operatorname{Ig} \lambda$-chain $C(2.0,243)$, protein NEL $(2.3,162)$ and arginosuccinate synthase $(3.1,62)$ as compared to the AIL (Fig. 3CD and Supplementary Table S2B).

\subsection{The SF of RJF contained fewer immune system process proteins compared to WL and AIL}

The identified proteins were classified according to the 12 biological process categories for each breed of chicken (Fig. 4A and Table 2A). The highest number of proteins in the SF of RJF, WL and AIL were found in the metabolic process category (10, 8 and 13 respectively) while in the 'immune system process' category 3, 5 and 6 proteins were identified, respectively. The protein class analysis revealed 11, 15, and 18 types of proteins, respectively, in the seminal fluid of RJF, WL and AIL (Fig. 4B and Table 2B). Proteins were also screened in the EMBL-EBI Quick GO database to ascertain if any one of them were from sperm regions of Gallus gallus. We found that the trypsin inhibitor ClTl-1 (ISK1L_CHICK) is an acrosome membrane protein (GO: 0002080) and was detected in the SF of each breed.

\subsection{The seminal fluid of RJF, WL and AIL contain TGF-ß2 and CXCL10}

The Luminex screening solely detected TGF- $\beta 2$ (RJF, 14810.47 \pm 1492.56 ; WL, 17111.14 \pm 1028.78; AIL, 13706.36 \pm 1318.50 , pg/ml, mean \pm SEM, Fig. 5A) and CXCL10 (RJF, 48.74 \pm 5.75 ; WL, 0.00; AIL, 56.65 \pm 14.93 , pg/ml, mean \pm SEM, Fig. 5B). The other cytokines or chemokines (IFN $\gamma$, CCL22, CXCL1, IL-17, GM-CSF, CCL2, IL-6, CXCL8, IL-15, IL-10, TGF$\beta 1$ and TGF- $\beta 3$ ) were not in the detectable range when measured by Luminex. The following use of a chicken specific ELISA could not confirm the presence of TGF- $\beta 2$ in RJF but could on WL 


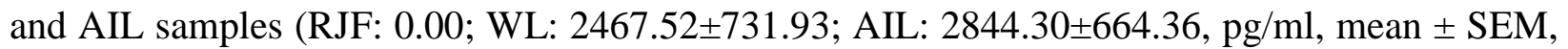
Fig. 5C). Regarding CXCL10, the ELISA confirmed its presence in the SF of all breeds in contrast to the Luminex (RJF: 107.65 \pm 16.40 ; WL: 71.54 \pm 5.97 ; AIL: 86.46 $\pm 7.36, \mathrm{pg} / \mathrm{ml}$, mean \pm SEM, Fig. 5D). ELISA measurements depicted further differences between breeds; thus, while TGF- $\beta 2$ levels did not differ between WL and AIL, the cytokine was absent in RJF. The concentrations of CXCL10 differed significantly between RJF and WL $(\mathrm{P}<0.05)$ with the SF of AIL having intermediate concentrations (n.s.).

\section{Discussion}

As it is the case in mammals, rooster seminal fluid has been considered to be a modulator of sperm functions, including fertilization (Douard et al., 2005; Froman et al., 2011; Marzoni et al., 2013). While even seminal plasma in mammals has been ascribed relevant roles in fertility including that of livestock selected for fertility, as pigs (Robertson, 2005; Song et al., 2016) such counterstudies are not available for chicken. Selection for fertility, marking differences in egglaying capacity seemed to have affected sperm function. Murugesan et al. (2013) found high egg laying WL-lines had poorer sperm concentration and forward sperm motility than low-egg laying WL-lines. Uncertainty prevails, since other studies did not found any such differences (Frankham and Doornenbal, 1972; Niranjan et al., 2001). Progressive sperm motility appeared significantly higher in RJF compared to the domestic bantam chicken (Malik et al., 2013). Whether comparative changes have occurred in SF-components and whether eventual changes affect the endowement that SF provides the ejaculated spermatozoa with, as it is proven for mammals (Rodriguez-Martinez et al., 2011; Perez-Patiño et al., 2016) is yet to be explored. Chicken spermatozoa are stored for days to weeks in the sperm-storage oviduct area (UVJ). Since spermatozoa (and the SF) are foreign to the hen, some sort of negotiation with the female immune system must be achieved to allow such long survival, with maintenance of potential fertilizing capacity. In mammals, the seminal plasma induces a state of immune tolerance (Robertson et al., 2005; Rodriguez-Martinez et al., 2011). Mating is capable of inducing changes in the expression of genes at the sperm storage region in the chicken oviduct, including genes involved in immune functions (Atikuzzaman et al., 2015), indicating that similar mechanisms might be present in either animal classes. Ongoing studies replacing mating for infusion of seminal fluid in RJF and WL and exploring the utero-vaginal junction (UVJ) containing spermstorage tubuli (SST) by oligonucleotide microarray has shown changes in the expression of immune-responsive genes (7 genes in RJF and 9 genes in WL) in this specific area of the oviduct (Atikuzzaman et al., unpublished results). The capacity of the seminal fluid to modulate immune responsive genes would therefore imply that a differential protein abundance in the seminal fluid might have a regulating capacity of the oviductal sperm storage area by modulating the local immune responsive genes. If so, selection might have changed this capacity, not affecting the spermatozoa but the SF proteome. Further studies are of course needed to explore this possibility. Differences in SF-proteins between the low egg-laying progenitor RJF, the high egglaying modern domestic WL and an AIL- intercross between the two, are hereby made evident.

4.1 The SF of RJF lacks astacin-like metalloendopeptidase which is commonly found in most biological process and protein class categories 
The present study showed that the numbers of identified SF-proteins from RJF, in the biological process categories and protein classes, were closer to WL, yet less than in the AIL (Fig. 4 and Table 2). Among the biological process categories, the RJF-SF contained stimulus responsive proteins (gallinacin-9 and apolipoprotein A-l) maintained in WL and AIL (Table 2A). However, the RJF-SF lacked several stimulus responsive proteins that were only detected in the SF of WL (i.e. astacin-like metalloendopeptidase) or AIL (i.e. astacin-like metalloendopeptidase, coagulation factor IX and thioredoxin). Astacin-like metalloendopeptidase, relevant in most of the biological processes and protein class categories (Table 2), is present in the seminal fluid of D. melanogaster, and is responsible for processing other reproductive proteins and for inducing a variety of post-mating responses in the female fly (Ram et al., 2006; Sirot et al., 2009; Ayroles et al., 2011; LaFlamme et al., 2012). Our results suggest this protein is also important in the chicken, reinforcing results previously reported (Labas et al., 2015). Interestingly, this protein was apparently absent in the SF of the ancestor RJF, calling for further studies to determine whether evolution has elevated the levels of this particular protein in modern chicken. Coagulation factor IX is an anti-hemophilic B single chain polypeptide with $\mathrm{M}_{\mathrm{r}}$ 57,000 Da in human and bovine which circulates in blood plasma (DiScipio et al., 1978; Katayama et al., 1979). In humans, the coagulation factor IX has been detected in the seminal plasma of both fertile and unfertile donors (Lwaleed et al., 2005). However, further research is necessary to disclose whether coagulation factor IX could be a biomarker for rooster fertility. Thioredoxin has been reported in the Gallus gallus seminal fluid being classified as defense- or immunity-protein (Marzoni et al., 2013) while in the present study it was classified as a stimulus responsive protein (Table 2A). Cytoskeletal proteins especially tubulins are strongly expressed in human normal sperm compared to sperm from infertile man (Salvolini et al., 2013). In the present study, cytoskeletal proteins were only detected in the AIL-SF, crossbred individuals without fertility deficiencies. Further analyses are needed to explore this exception.

4.2 The rooster seminal fluid conserves serum albumin and ovotransferrin, but they are overexpressed in modern WL and AIL, suggesting a role for production performance

There were a number of proteins conserved in modern chicken. Around fifty percent of the identified proteins in the seminal fluid of all breeds from the 2D gels were ovotransferrin and serum albumin (Table 1), confirming recent reports (Castillo et al., 2011; Marzoni et al., 2013). Serum albumin (spots 126 and 214) was differentially expressed (with lesser expression in the RJF than in WL and AIL) (Fig. 3 and Supplementary Table 2). Serum albumin reported in both chicken (Marzoni et al., 2013) and sheep (Soleilhavoup et al., 2014), originates from the epididymis and prostate gland in humans (Elzanaty et al., 2007) but its origin is not determined as yet in chicken. Serum albumin has been classified as a defense or immunity protein (Marzoni et al., 2013) and it is used as an additive in animal semen handling industry (Matsuoka et al., 2006; Fukui et al., 2007; Hossain et al., 2007; Xia and Ren, 2009; Nang et al., 2012), since it appears to increase the percentage of motile spermatozoa and sperm velocity (Bakst and Cecil, 1992). The overall consideration is that the different expression levels between ancestors and modern breeds might relate to their documented differences in fertility.

Ovotransferrin (Spots 123 and 192), a member of iron-binding transferrin and metalloproteinases, has antimicrobial activity (Valenti et al., 1983) and is found in all roosters 
yet in lesser quantities (2 to 4 fold) in the SF of RJF than in that of WL and the AIL (Fig. 3 and Supplementary Table 2). In mammalian seminal plasma, transferrin originates in the Sertoli cells (Holmes et al., 1982; Gilmont et al., 1990) and thus been shown to be an important biomarker for spermatogenesis (Holmes et al., 1982; Bharshankar and Bharshankar, 2000). Ovotransferrin as a defensive protein (Marzoni et al., 2013) is also present in egg albumin, depicting a strong bactericidal effect (Baron et al., 2014). It is therefore possible that during chicken mating, the ovotransferrin contained in the SF acts as an antimicrobial during semen deposition in the female cloaca, thus decreasing pathogen overload during sperm colonization and storage in the oviduct reservoir (sperm-storage tubules). A lower amount of the protein in RJF-SF as compared to WL- and AIL- might therefore be related to production performance.

Another conserved protein is the cytoskeletal protein-gelsolin family, present in all roosters albeit in different types (Gelsolin, Src substrate protein p85, tubulin $\alpha-5$ chain, tubulin $\beta-1$ chain and tubulin $\beta$-5 chain) (Table 2B). Among these cytoskeletal proteins, Gelsolin is reported as influencing the acrosome reaction during human fertilization (Finkelstein et al., 2010). Although it is unclear what role this protein plays in chicken fertilization, it is interesting that it is conserved in such a primitive phenomenon as internal fertilization.

4.3 Other differentially expressed proteins in the seminal fluid might mirror egg-laying capacity evolved through selection pressure

In addition to serum albumin and ovotransferrin, ovoinhibitor (spot 129), creatinine kinase Btype (spot 131), $\alpha$-enolase (spot 132), L-lactate dehydrogenase B chain (spot 140), triosephosphate isomerase (spot 145), retinol-binding protein (spot 146), apolipoprotein A-I (spot 147), malate dehydrogenase (spot 139), glyceraldehyde-3-phosphate dehydrogenase (spot 141), cystatin (spot 152) and gallinacin-9 (spot 157) were differentially expressed in the SF of RJF compared to WL, whereas, phosphoglycerate mutase 1 (spot 230), tubulin beta-1 chain (spot 172), transthyretin (spot 85), Ig $\lambda$-chain $C$ (spot 243), protein NEL (spot 162) and arginosuccinate synthase (spot 62) were differentially expressed in the SF of RJF while compared with AIL (Fig. 3 and Supplementary Table 2).

Among these differentially expressed SF proteins between RJF and WL, ovoinhibitor, creatinine kinase B-type, $\alpha$-enolase, L-lactate dehydrogenase B-chain, triosephosphate isomerase, retinol binding protein and apolipoprotein A-l were down-expressed in RJF compared to WL, whereas, glyceraldehyde-3-phosphate dehydrogenase, cystatin and gallinacin-9 were over-expressed in RJF compared to WL. Ovoinhibitor was reported as having antibacterial activity in turkey seminal fluid (Slowinska et al., 2014). Creatinine kinase B-type, $\alpha$-enolase, triosephosphate isomerase and glyceraldehyde-3-phosphate dehydrogenase are reported chicken seminal fluid proteins, classified as energy metabolism proteins (Marzoni et al., 2013). This author also reported apolipoprotein A-l as a transport or binding protein and gallinacin- 9 as a defense or immunity protein in chicken-SF. Malate dehydrogenase was reported to be 2.23 fold increased in the SF of infertile chicken (Labas et al., 2015). Our findings of down-expression of proteins with bactericidal (ovoinhibitor), sperm motility-enhancing (creatinine kinase B-type), energy metabolism ( $\alpha$-enolase and triosephosphate isomerase) and binding (apolipoprotein A-l) capacities; as well as the over-expression of defense or immunity protein (gallinacin-9) in the SF 
of low-laying RJF compared to high-laying WL (Fig. 3A and Supplementary Table S2A) suggest these proteins relate to their differential fertility.

Phosphoglycerate mutase 1 and tubulin $ß-1$ chain are down-expressed in the SF of RJF compared to AIL (Fig. 2C and Supplementary Table S2B). Phosphoglycerate mutase 1 has been categorized into energy metabolism group (Marzoni et al., 2013) has also been identified in rooster sperm (Labas et al., 2015). In ruminant seminal plasma (bull, buck and ram) however, only phosphoglycerate mutase 2 has been identified (Druart et al., 2013). In a more recent report (Labas et al., 2015), predicted tubulin alpha-3 and tubulin $\$-3$ chains were detected in rooster sperm while tubulin $ß-2 \mathrm{C}$, isoform CRA-b was detected in the seminal plasma of rams (Soleilhavoup et al., 2014), indicating that differences are present between these classes. Four proteins (transthyretin, Ig $\lambda$-chain $\mathrm{C}$, protein NEL and arginosuccinate synthase) were found over-expressed in the seminal fluid of RJF compared with AIL (Fig. 3C and Supplementary Table S2B). Transthyretin was found overexpressed in the yellow seminal fluid of sub-fertile turkey compared to fertile males (Slowinska et al., 2015). The Ig $\lambda$-chain C-a defense/immunity protein (Table 2), previously reported in rooster-SF (Labas et al., 2015), and protein NEL- is a developmental process and calcium ion-binding protein (Table 2A) found in bovine seminal plasma (Kelly et al., 2006)- were over-expressed in RJF-SF compared to AIL-SF, in the current study, albeit reported before as down-expressed in infertile roosters (Labas et al., 2015). Argininosuccinate synthase seems to be involved in disposing excess ammonium in semen (Dietz and Flipse, 1969), and related to nitric oxide metabolism. However, it is not known whether the increment of this protein, as found in the present study, relates the enzyme to sperm survival in neither ancestor nor modern chicken. Its character as biomarker for sperm quality warrants further studies of this enzyme.

4.4 The seminal fluid of RJF lacks TGF- $\beta 2$ but is rich in CXCL10: a relation to lower sperm survival?

Cytokines and chemokines are important modulators of the immune system process. They are necessary to help the female eliminate pathogens entering with the semen but at the same time allow spermatozoa to survive in the sperm reservoir at the utero-vaginal junction (UVJ), as it happens in the mammalian tubal sperm reservoirs (Robertson et al., 2002). A bead-based immune-assay screening of a battery of cytokines/chemokines reported in mammals (Barranco et al., 2015) was initially done, which resulted in only few cytokines being detectable in rooster SF, i.e. the immune-suppressive TGF- $\beta$ and the immune cell-chemoattractant CXCL10, those related to the above mentioned dual effects (Agostini et al., 2001; Dufour et al., 2002; Das et al., 2006). Since this initial detection in SF was not present in all breeds, a new attempt was done to confirm their presence by using a chicken-specific ELISA, where we could find that TGF- $\beta 2$ levels did not differ between WL and AIL but that the cytokine was absent in the SF of RJF (Fig. 5C). The concentrations of CXCL10 differed significantly between RJF and WL $(\mathrm{P}<0.05)$ with AIL values in between (Fig. 5D). The cytokine TGF- $\beta 2$ is present in the seminal plasma of normal fertile men (Nocera and Chu, 1995; Srivastava et al., 1996). The presence of TGF $\beta$ in the seminal fluid is usually in latent form therefore, has to be activated in the female reproductive tract postinsemination (Robertson, 2005). In chicken, insemination increases the expression of TGF- $\beta 2$ in the UVJ, which is apparently responsible for the immunosuppression as well as it helps sperm 
survival (Das et al., 2006). In contrast, the chemokine CXCL10 is a chemoattractant that recruits activated T-cells (Agostini et al., 2001). CXCL10 ${ }^{-/-}$KO-mice show impaired T-cell responses (Dufour et al., 2002). Interestingly, the present study shows that immune-modulating cytokine/chemokine concentrations in the SF differ between low-laying and high-laying chicken breed, the RJF depicting high amounts of immune reacting CXCL10 and lack of the immunosuppressive cytokine-TGF- $\beta 2$. The modern domesticated chicken has evolved from this common ancestor RJF, towards a higher egg-laying capacity. Such selection has, apparently, contributed to differential SF-cytokines, which may be key determinants of sperm survival in the UVJ.

\section{Conclusion}

We are aware that the lack of orthogonal quantitative validation of the comparative 2DE data represents a potential weakness of the study. However, the current lack of commercial antibodies with proven specificity, and the absence of SILAC chicken model for quantitative proteomics, relegate orthogonal validation beyond the scope of this study. Consequently, our work should be seen as preliminary, and the quantitative validation of relevant proteins should be pursued in future studies. Given this limitation, our findings suggest that several proteins (especially gallinacin- 9 and Ig $\lambda$-chain C) and specific cytokines (TGF- $\beta 2$ and CXCL10) are differentially expressed in the SF between ancestor-RJF and modern- (WL and AIL) chicken, presumably due to the selection for production traits during chicken breeding.

\section{Conflict of interest statement}

The authors declare that there is no conflict of interest that could be perceived as prejudicing the impartiality of the research reported.

\section{Funding}

The project has been financed by the Research Council FORMAS, Stockholm, Sweden (Project number: 221-2011-512), and by grant BFU2013-42833-P from the Ministerio de Ciencia e Innovación (Madrid, Spain),

\section{Acknowledgements}

Prof. Jordi Altimiras and Per Jensen are acknowledged for putting at our disposal the chicken included in this study. We thank Prof. Maria Jenmalm for her suggestions regarding cytokine analyses.

\section{References}

Agostini C, Calabrese F, Rea F, Facco M, Tosoni A, Loy M, Binotto G, Valente M, Trentin L, and Semenzato G. CXCR3 and its ligand CXCL10 are expressed by inflammatory cells 
infiltrating lung allografts and mediate chemotaxis of $\mathrm{T}$ cells at site of rejection. Am J Pathol 158 (2001) 1703.

Atikuzzaman M, Bhai RM, Fogelholm J, Wright D, and Rodriguez-Martinez H. Mating induces the expression of immune- and $\mathrm{pH}$-regulatory genes in the utero-vaginal junction containing mucosal sperm-storage tubuli of hens. Reproduction 150 (6) (2015) 473-83.

Ayroles JF, LaFlamme BA, Stone EA, Wolfner MF, and Mackay TF. Funtional genome annotation of Drosophila seminal fluid proteins using transcriptional genetic networks. Genet Res 93 (2011) 387-395.

Bakst MR, and Cecil HC. Effect of bovine serum albumin on motility and fecundity of turkey spermatozoa before and after storage. J Reprod Fertil 94 (1992) 287-293.

Baron F, Jan S, Gonnet F, Pasco M, Jardin J, Giudici B, Gautier M, Guérin-Dubiard C, and Nau F. Ovotransferrin plays a major role in the strong bactericidal effect of egg white against the Bacillus cereus group. J Food Prot 77(6) (2014) 955-62.

Barranco I, Rubér M, Perez-Patiño C, Atikuzzaman M, Martinez EA, Roca J, RodriguezMartinez H. The seminal plasma of the boar is rich in cytokines, with significant individual and intra-ejaculate variation. Am J Reprod Immunol 74 (2015) 523-532.

Batruch I, Lecker I, Kagedan D, Smith CR, Mullen BJ, Grober E, Lo KC, Diamandis EP, and Jarvi KA. Proteomic analysis of seminal plasma from normal volunteers and postvasectomy patients identifies over 2000 proteins and candidate biomarkers of the urogenital system. J Proteome Res 10 (2011) 941-953.

Bharshankar RN, Bharshankar JR. Relationship of seminal plasma transferrin with seminal parameters in male infertility. Indian J Physiol Pharmacol 44(4) (2000) 456-60.

Blesbois $\mathrm{E}$ and Hermier D. Effects of high-density lipoproteins on storage at $4^{0} \mathrm{C}$ of fowl spermatozoa. J Reprod Fertil 90 (1990) 473-482.

Bromfield JJ, Schjenken JE, Chin PY, Care AS, Jasper MJ, and Robertson SA. Maternal factors contribute to paternal seminal fluid impact on metabolic phenotype in offspring. Proc Natl Acad Sci 111 (2014) 2200-2205.

Caballero I, Parrilla I, Almiñana C, del Olmo D, Roca J, Martínez EA, and Vázquez JM. Seminal plasma proteins as modulators of the sperm function and their application in sperm biotechnologies. Reprod Domest Anim 47 (Suppl.3) (2012) 12-21.

Calvete JJ, Fasoli E, Sanz L, Boschetti E, and Righetti PG. Exploring the venom proteome of the Western Diamondback Rattlesnake, Crotalus atrox, via snake venomics and combinatorial peptide ligand library approaches. J Proteome Res 8 (2009) 3055-3067.

Calvete JJ, Nessau S, Mann K, Sanz L, Sieme H, Klug E, and Töpfer-Petersen E. Isolation and biochemical characterization of stallion seminal-plasma proteins. Reprod Domest Anim 29 (1994) 411-426.

Castillo A, Marzoni M, Sagona S, Citti L, Rocchiccioli S, Romboli I, and Felicioli A. Ovotransferrin and gallinacin-9 found in seminal plasma rooster. $6^{\text {th }}$ ITPA Annual National Conference, Torino (2011).

Crawford G, Ray A, Gudi A, Shah A, and Homburg R. The role of seminal plasma for improved outcomes during in vitro fertilization treatment: review of the literature and metaanalysis. Hum Reprod Update 21 (2015) 275-284. 
Das SC, Isobe $\mathrm{N}$, and Yoshimura $\mathrm{Y}$. Changes in the expression of interleukin-1 $\beta$ and lipopolysaccharide-induced TNF factor in the oviduct of laying hens in response to artificial insemination. Reproduction 137 (2009) 527-536.

Das SC, Isobe N, and Yoshimura Y. Expression of Toll-like receptors and avian b-defensins and their changes in response to bacterial components in chicken sperm. Poult Sci 90 (2011) 417-425.

Das SC, Isobe N, and Yoshimura Y. Mechanisms of prolonged sperm storage and sperm survivality in hen oviduct: A review. Reprod Immunol 60 (2008) 477-481.

Das SC, Isobe N, Nishibori M, and Yoshimura Y. Expression of TGF $\beta$-isoforms and their receptors in utero-vaginal junction of hen oviduct in presence or absence of resident sperm with reference to sperm storage. Reproduction 132 (2006) 781-790.

Dietz RW, and Flipse RJ. Metabolism of bovine semen. XX. Role of ammonia in interactions between the citric acid and urea cycles. Biol Reprod 1(1969) 200-206.

DiScipio RG, Kurachi K, and Davie EW. Activation of human factor IX (Christmas factor). J Clin Invest 61 (1978) 1528-1538.

Douard V, Hermier D, Labbe C, Magistrini M, and Blesbois E. Role of seminal plasma in damage to turkey spermatozoa during in vitro storage. Theriogenology 63 (2005) 126137.

Druart X, Rickard JP, Mactier S, Kohnke PL, Kershaw-Young CM, Bathgate R, Gibb Z, Crossett B, Tsikis G, Labas V, Harichaux G, Grupen CG, and de Graaf SP. Proteomic characterization and cross species comparison of mammalian seminal plasma. J Proteomics 91 (2013) 13-22.

Dufour JH, Dziejman M, Liu MT, Leung JH, Lane TE, and Luster AD. IFN- $\gamma$-inducible protein 10 (IP-10; CXCL10)-deficient mice reveal a role for IP-10 in effector T cell generation and trafficking. J Immunol 168 (2002) 3195-3204.

Elzanaty S, Erenpreiss J, and Becker C. Seminal plasma albumin: origin and relation to the male reproductive parameters. Andrologia 39 (2007) 60-65.

Etches RJ. The male. In: Etches, RJ (Ed.), Reproduction in poultry. CAB International, Oxford (1996) 208-233.

Fazeli A, Affara NA, Hubank M, and Holt WV. Sperm-induced modification of the oviductal gene expression profile after natural insemination in mice. Biol Reprod 71 (2004) 60-65.

Finkelstein M, Etkovitz N, and Breitbart H. Role and regulation of sperm gelsolin prior to fertilization. J Biol Chem 285 (2010) 39702-39709.

Frankham F, Doornebal H. Semen characteristics of lines selected for increased part-record egg production. Poult Sci 51 (1972) 1468-1469.

Froman DP, Feltmann AJ, Pendarvis K, Cooksey AM, Burgess SC, and Rhoads DD. A proteome-based model for sperm mobility phenotype. J Anim Sci 89 (2011) 1330-1337.

Fujihara N. Accessory reproductive fluids and organs in male domestic birds. Worlds Poult Sci J 48 (1992) 39-56.

Fukui Y, Kohno H, Togari T, and Hiwasa M. Fertility of ewes inseminated intrauterinally with frozen semen using extender containing bovine serum albumin. J reprod Dev 53 (2007) 959-962.

Gilmont RR, Senger PL, Sylvester SR, and Griswold MD. Seminal transferrin and spermatogenic capability in the bull. Biol Reprod 43 (1990) 151-157. 
Harris GC Jr and Sweeney MJ. Changes in the protein concentration of chicken seminal plasma after rapid freeze-thaw. Cryobiology 7 (1971) 209-215.

Holmes SD, Lipshultz LI, and Smith RG. Transferrin and gonadal dysfunction in man. Fertil Steril 38 (1982) 600-604.

Hossain MS, Hyeong LJ, Miah AG, and Tsujii H. Effect of fatty acids bound to bovine serum albumin-V on acrosome reaction and utilization of glucose in boar spermatozoa. Reprod Med Biol 6 (2007) 109-115.

Johnsson M, Gustafson I, Rubin C-J, Sahlqvist A-S, Johnsson KB, Kerje S, Ekwall O, Käampe $\mathrm{O}$, Andersson L, Jensen P \& Wright D. A sexual ornament in chickens is affected by pleiotropic alleles at HAO1 and BMP2, selected during domestication. PLoS Genet 8 (2012) e1002914.

Kareskoski AM, Rivera del Alamo MM, Güvenc K, Reilas T, Calvete JJ, Rodriguez-Martinez H, Andersson M, and Katila T. Protein composition of seminal plasma in fractionated stallion ejaculates. Reprod Domest Anim 46 (2011) e79-e84

Katayama K, Ericsson LH, Enfield DL, Walsh KA, Neurath H, Davie EW, and Titani K. Comparison of amino acid sequence of bovine coagulation factor IX (Christmas factor) with that of other vitamin K-dependant plasma proteins. Proc Natl Acad Sci 76 (1979) 4990-4994.

Kelly VC, Kuy S, Palmer DJ, Xu Z, Davis SR, and Cooper GJ. Characterization of bovine seminal plasma by proteomics. Proteomics 6 (2006) 5826-5833.

Labas V, Grasseu I, Cahier K, Gargaros A, Harichaux G, Teixeira-Gomes A-P, Alves S, Bourin M, Gérard N, and Blesbois E. Qualitative and quantitative peptidomic and proteomic approaches to phenotyping chicken semen. J Proteomics 112 (2015) 313-335.

Laemmli, UK. Cleavage of structural proteins during the assembly of the head of bacteriophage T4. Nature 227 (1970) 680-685.

LaFlamme BA, Ram KR, and Wolfner MF. The Drosophila melanogaster seminal fluid protease “seminase” regulates proteolytic and post-mating reproductive process. PLoS Genet 8 (2012) e1002435.

Lwaleed BA, Greenfield RS, Hicks J, Birch BR, and Cooper AJ. Quantitation of seminal factor IX and factor IXa in fertile, nonfertile, and vasectomy subjects: a step closer toward identifying a functional clotting system in human semen. J Androl 26 (2005) 146-152.

Malik A, Haron AW, Yosoff R, Nesa M, Bukar M, Kasim A. Evaluation of the ejaculate quality of the red jungle fowl, domestic chicken, and bantam chicken in Malaysia. Turkish J Vet Anim Sci 37 (2013) 564-568.

Mann T, Mann CL, and Dixon RL. Passage of chemicals into human and animal semen: mechanisms and significance. Crit Rev Toxicol 11(1) (1982) 1-14.

Marzoni M, Castillo A, Sagona S, Citti L, Rocchiccioli S, Romboli I, and Felicioli A. A proteomic approach to identify seminal plasma proteins in roosters (Gallus gallus domesticus). Anim Reprod Sci 140 (2013) 216-223.

Matsuoka T, Imai H, Kohno H, and fukui Y. Effects of bovine serum albumin and trehalose in semen diluents for improvement of frozen-thawed ram spermatozoa. J Reprod Dev 52 (2006) 675-683. 
Mi H, Poudel S, Casagrande AMJT, and Thomas PD. PANTHER version10: expanded protein families and functions, and analysis tools. Nucleic Acids Res 44 (D1) (2016) D336-42 doi:10.1093/nar/gkv1194.

Michailidis G, Anastasiadou M, Guibert E, and Froment P. Activation of innate immune system in response to lipopolysaccharide in chicken sertoli cells. Reproduction 148 (2014) 259270.

Milardi D, Grande G, Vincenzoni F, Castagnola M, and Marana R. Proteomics of human seminal plasma: identification of biomarkers candidates for fertility and infertility and the evolution of technology. Mol Reprod Dev 80 (2013) 350-357.

Murugesan S, Matam N, Kulkarni R, Bhattacharya TK, Chatterjee R. Semen quality in white leghorn chicken selected for egg production traits. Turk J Vet Anim Sci 37 (2013) 747749.

Nang CF, Osman K, Budin SB, Ismail MI, Jaffar FHF, Mohamad SFS, and Ibrahim SF. Bovine serum albumin: survival and osmolarity effect in bovine spermatozoa stored above freezing point. Andrologia 44 (2012) 447-453.

Niranjan M, Datt C, Das A. Semen quality studies in two White Leghorn lines maintained at Tripura. Indian J Poult Sci 36 (2001) 287-289.

Nocera M, Chu TM. Characterization of latent transforming growth factor-beta from human seminal plasma. Am J Reprod Immunol 33 (1995) 282-291.

Novak S, Ruiz-Sánchez A, Dixon WT, Foxcroft GR, and Dyck MK. Seminal plasma proteins as potential markers of relative fertility in boars. J Androl 31 (2010) 188-200.

Ocón-Grove OM, Krzysik-Walker SM, Maddineni SR, Hendricks II GL, and Ramachandran R. NAMPT (visfatin) in the chicken testis: influence of sexual maturation on cellular localization, plasma levels and gene and protein expression. Reproduction 139 (2010) 217-226.

Perez-Patiño C, Barranco I, Parrilla I, Martinez EA, Rodriguez-Martinez H, and Roca J. Characterization of the porcine seminal plasma proteome comparing ejaculate portions. $\mathrm{J}$ Proteomics 142 (2016) 15-23.

Pilch B and Mann M. large-scale and high-confidence proteomic analysis of human seminal plasma. Genome Biol 7 (2006) R40.

Ram KR, Sirot LK, and Wolfner MF. Predicted seminal astacin-like protease is required for processing of reproductive proteins in Drosophila melanogaster. Proc Natl Acad Sci 103 (2006) 18674-18679.

Robertson SA, Ingman WV, O’Leary S, Sharkey DJ, Tremellen KP. Transforming growth factor $\beta$-a mediator of immune deviation in seminal plasma. J Reprod Immunol 57 (2002) 109128.

Robertson SA. Seminal plasma and male factor signaling in the female reproductive tract. Cell Tissue Res 322 (2005) 43-52.

Rodrigues MAM, Souza CEA, Martins JAM, Rego JPA, Oliveira JTA, Domont G, Nogueira FCS, and Moura AA. Seminal plasma proteins and their relationship with sperm motility in Santa Ines rams. Small Ruminant Res 109 (2013) 94-100.

Rodríguez-Martínez H, Iborra I, Martínez P, and Calvete JJ. Immunoelectroscopic imaging of speradhesin AWN epitopes on boar spermatozoa bound in vivo to the zona pellucida. Reprod Fertil Dev 10 (1998) 491-497. 
Rodríguez-Martínez H, Kvist U, Ernerudh J, Sanz L, and Calvete JJ. Seminal plasma proteins: what role do they play? Am J Reprod Immunol 66 (Suppl. 1) (2011) 11-22.

Rodríguez-Martínez H, Saravia F, Wallgren M, Roca J, and Pena FJ. Influence of seminal plasma on kinematics of boar spermatozoa during freezing. Theriogenology 70 (2008) 1242-1250.

Salvolini E, Buldreghini E, Lucarini G, Vignini A, Lenzi A, Di Primio R, and Balercia G. Involvement of sperm plasma membrane and cytoskeletal proteins in human male infertility. Fertil Steril 99 (2013) 697-704

Schjenken JE, and Robertson SA. Seminal fluid and immune adaptation for pregnancycomparative biology in maternal species. Reprod Domest Anim 49 (Suppl. 3) (2014) 2736.

Sharma R, Agarwal A, Mohanty G, Jesudasan R, Gopalan B, Willard B, Yadav SP, and Sabanegh E. Functional proteomic analysis of seminal plasma proteins in men with various semen parameters. Reprod Biol Endocrinol 11 (2013) 38.

Sirot LK, LaFlamme BA, Sitnik JL, Rubinstein CD, Avila FW, Chow CY, and Wolfner MF. Molecular social interactions: Drosophila melanogaster seminal fluid proteins as a case study. Adv Genet 68 (2009) 23-56.

Slowin'ska M, Liszewska E, Nynca J, Bukowska J, Hejmej A, Bilin’ska B, Szubstarski J, Kozlowski K, Jankowski J, and Ciereszko A. Isolation of an ovoinhibitor, a multidomain kazal-like inhibitor from turkey (Meleagris gallopavo) seminal plasma. Biol Reprod 91(5) (2014) 108.

Slowinska M, Kozlowski K, Jankowski J, and Ciereszko A. Proteomic analysis of white and yellow seminal plasma in turkeys. J Anim Sci 93(6) (2015) 2785-95.

Soggiu A, Piras C, Hussein HA, De Canio M, Gaviraghi A, Galli A, Urbani A, Bonizzi L, and Roncada P. Unravelling the bull fertility proteome. Mol Biosyst 9 (2013) 1188-1195.

Soler L, Labas V, Thélie A, Grasseau I, Teixeira-Gomes A-P, and Blesbois E. Intact cell MALDI-TOF MS on sperm: a molecular test for male fertility. Mol Cell Proteomics 15 (2016) 1998-2010.

Soleilhavoup C, Tsikis G, Labas V, Harichaux G, Kohnke P, Dacheux J-L, Guérin Y, Gatti J-L, De Graaf SP, Druart X. Ram seminal plasma proteome and its impact on liquid preservation of spermatozoa. J Proteomics 109 (2014) 245-260.

Song Z-H, Li Z-Y, Li D-D, Fang W-N, Liu H-Y, Yang D-D, Meng C-Y, Yang Y, and Peng J-P. Seminal plasma induces inflammation in the uterus through the $\gamma \delta \mathrm{T} / \mathrm{IL}-17$ pathway. Sci Rep 6 (2016) 25118.

Srivastava MD, Lippes J, Srivastava BL. Cytokines of the human reproductive tract. Am J Reprod Immunol 36 (1996) 157-166.

Thurston RJ, Hess RA, Froman DP, Biellier HV. Elevated seminal plasma protein: a characteristic of yellow turkey semen. Poult Sci 61(9) (1982) 1905-11.

Valenti P, Antonini G, Von Hunolstein C, Visca P, Orsi N, and Antonini E. Studies of the antimicrobial activity of ovotransferrin. Int J Tissue React 5 (1) (1983) 97-105.

Xia $\mathrm{J}$ and Ren D. The BSA-induced $\mathrm{Ca}(2+)$ influx during sperm capacitation is CATSPERM channel-dependent. Reprod Biol Endocrinol 7 (2009) 119. 
Table 1. Comparative identified proteins in chicken seminal fluid detected by 2D SDS-PAGE followed by mass spectrometry. Total protein spots on 2D gels -107 of RJF, 52 of WL and 98 of AIL- were analysed using LC-ESI-QTOF-MS/MS. Percent calculated from total number of spots analyzed in each breed.

\begin{tabular}{|c|c|c|c|c|}
\hline \multirow[t]{2}{*}{$\begin{array}{l}\text { Proteins identified in the chicken } \\
\text { seminal fluid }\end{array}$} & \multicolumn{3}{|c|}{$\begin{array}{l}\text { Proteins detected in the seminal fluid } \\
\% \text { (total number of spots identified for a protein) }\end{array}$} & \multirow[t]{2}{*}{ Spot number(s) on acrylamide gels } \\
\hline & RJF & WL & AIL & \\
\hline Aspartate aminotransferase, cytoplasmic & $1.87(2)$ & $0(0)$ & $0(0)$ & 71,72 \\
\hline $\begin{array}{l}\text { Angiotensin-converting enzyme } \\
\text { (Fragment) }\end{array}$ & $0(0)$ & $0(0)$ & $1.02(1)$ & 161 \\
\hline Serum albumin & $11.21(12)$ & $21.15(11)$ & $13.27(13)$ & $\begin{array}{l}2,4,7,9,10,12,14,16,42,51,52,53, \\
108,109,111,112,113,114,116,117, \\
124,125,126,169,170,171,182,188, \\
195,196,199,212,213,214,217,246\end{array}$ \\
\hline Apolipoprotein A-I & $1.87(2)$ & $5.78(3)$ & $4.08(4)$ & $\begin{array}{l}80,90,142,143,147,237,241,242, \\
244\end{array}$ \\
\hline Annexin A5 & $0.93(1)$ & $0(0)$ & $0(0)$ & 86 \\
\hline Argininosuccinate synthase & $0.93(1)$ & $0(0)$ & $0(0)$ & 62 \\
\hline Astacin-like metalloendopeptidase & $0(0)$ & $1.92(1)$ & $1.02(1)$ & 141,252 \\
\hline $\begin{array}{l}\text { Complement factor B-like protease } \\
\text { (Fragment) }\end{array}$ & $0(0)$ & $1.92(1)$ & $1.02(1)$ & 120,184 \\
\hline Cystatin & $0.93(1)$ & $1.92(1)$ & $3.06(3)$ & $100,152,248,250,251$ \\
\hline Alpha-enolase & $1.87(2)$ & $1.92(1)$ & $1.02(1)$ & $57,66,132,222$ \\
\hline Beta-enolase & $0.93(1)$ & $0(0)$ & $1.02(1)$ & 66, 222 \\
\hline Gamma-enolase & $0(0)$ & $0(0)$ & $1.02(1)$ & 222 \\
\hline Coagulation factor IX & $0(0)$ & $0(0)$ & $1.02(1)$ & 169 \\
\hline Fatty acid-binding protein, brain & $1.87(2)$ & $1.92(1)$ & $3.06(3)$ & $97,98,149,247,248,249$ \\
\hline Fibrinogen alpha chain & $0(0)$ & $0(0)$ & $1.02(1)$ & 213 \\
\hline Fibrinogen beta chain (Fragment) & $1.87(2)$ & $0(0)$ & $4.08(4)$ & 50, 56, 210, 211, 212, 213 \\
\hline Gelsolin & $2.80(3)$ & $3.85(2)$ & $2.04(2)$ & $30,32,34,118,128,180,182$ \\
\hline Gallinacin-9 & $1.87(2)$ & $1.92(1)$ & $1.02(1)$ & $103,105,157,256$ \\
\hline Gallinacin-10 & $4.67(5)$ & $0(0)$ & $1.02(1)$ & $63,103,104,105,106,256$ \\
\hline Glutathione S-transferase 2 & $0.93(1)$ & $0(0)$ & $0(0)$ & 79 \\
\hline $\begin{array}{l}\text { Glyceraldehyde-3-phosphate } \\
\text { dehydrogenase }\end{array}$ & $0(0)$ & $1.92(1)$ & $0(0)$ & 141 \\
\hline Hemoglobin subunit alpha-D & $0(0)$ & $0(0)$ & $1.02(1)$ & 251 \\
\hline Ig mu chain $\mathrm{C}$ region & $1.87(2)$ & $5.77(3)$ & $2.04(2)$ & $6,31,114,116,117,182,188$ \\
\hline Ovoinhibitor & $2.80(3)$ & $1.92(1)$ & $4.08(4)$ & 45, 46, 47, 129, 206, 207, 208, 209 \\
\hline Trypsin inhibitor ClTI-1 & $1.87(2)$ & $3.85(2)$ & $2.04(2)$ & $101,102,154,155,253,254$ \\
\hline
\end{tabular}




\begin{tabular}{|c|c|c|c|c|}
\hline Creatine kinase B-type & $0.93(1)$ & $1.92(1)$ & $2.04(2)$ & $61,131,218,221$ \\
\hline Pyruvate kinase PKM & $0.93(1)$ & $0(0)$ & $1.02(1)$ & 54,204 \\
\hline Ig lambda chain $\mathrm{C}$ region & $3.74(4)$ & $1.92(1)$ & $4.08(4)$ & $87,88,89,90,142,239,241,242,243$ \\
\hline L-lactate dehydrogenase A chain & $0.93(1)$ & $0(0)$ & $0(0)$ & 75 \\
\hline L-lactate dehydrogenase B chain & $0.93(1)$ & $1.92(1)$ & $2.04(2)$ & $74,140,228,229$ \\
\hline Lysozyme C & $0(0)$ & $0(0)$ & $1.02(1)$ & 252 \\
\hline Malate dehydrogenase, cytoplasmic & $0.93(1)$ & $1.92(1)$ & $1.02(1)$ & $73,139,227$ \\
\hline Protein NEL & $0.93(1)$ & $0(0)$ & $3.06(3)$ & $3,161,162,170$ \\
\hline Phosphoglycerate mutase 1 & $0(0)$ & $0(0)$ & $1.02(1)$ & 230 \\
\hline Phosphoglycerate kinase & $0(0)$ & $0(0)$ & $1.02(1)$ & 225 \\
\hline Retinol-binding protein 4 & $0.93(1)$ & $1.92(1)$ & $1.02(1)$ & $94,146,244$ \\
\hline Src substrate protein p85 & $0(0)$ & $0(0)$ & $1.02(1)$ & 141 \\
\hline Tubulin alpha-5 chain & $0(0)$ & $0(0)$ & $2.04(2)$ & 172, 173 \\
\hline Tubulin beta- 1 chain & $0(0)$ & $0(0)$ & $1.02(1)$ & 172 \\
\hline Tubulin beta- 5 chain & $0(0)$ & $0(0)$ & $2.04(2)$ & 172,173 \\
\hline Thioredoxin & $0(0)$ & $0(0)$ & $1.02(1)$ & 246 \\
\hline Triosephosphate isomerase & $1.87(2)$ & $1.92(1)$ & $1.02(1)$ & $79,81,145,232$ \\
\hline Ovotransferrin & $15.89(17)$ & $17.31(9)$ & $12.24(12)$ & $\begin{array}{l}16,17,18,26,27,28,31,32,34,35, \\
36,37,38,39,41,52,102,114,116, \\
117,118,119,120,121,122,123,181, \\
185,188,189,191,192,193,194,201, \\
203,235,252\end{array}$ \\
\hline Transthyretin & $3.74(4)$ & $3.85(2)$ & $4.08(4)$ & $\begin{array}{l}84,85,96,97,134,148,211,237,238, \\
245\end{array}$ \\
\hline
\end{tabular}


Table 2A. Biological process analysis of identified protein in the seminal fluid of Red Junglefowl (RJF), White Leghorn (WL) and Advanced Intercross Line (AIL) male chicken.

\begin{tabular}{|c|c|c|c|c|}
\hline \multirow{2}{*}{\multicolumn{2}{|c|}{ Category name (Accesssion) }} & \multicolumn{3}{|c|}{ (Number of protein identified) Identified protein symbols } \\
\hline & & RJF & WL & AIL \\
\hline 1 & $\begin{array}{l}\text { Cellular component } \\
\text { organization or biogenesis } \\
\text { (GO:0071840) }\end{array}$ & $\begin{array}{c}\text { (2) } \\
\text { Gelsolin } \\
\text { Apolipoprotein A-I }\end{array}$ & $\begin{array}{c}\text { (2) } \\
\text { Gelsolin } \\
\text { Apolipoprotein A-I }\end{array}$ & $\begin{array}{c}\text { (3) } \\
\text { Gelsolin } \\
\text { Src substrate protein p85 } \\
\text { Apolipoprotein A-I }\end{array}$ \\
\hline 2 & $\begin{array}{l}\text { Cellular process } \\
\text { (GO:0009987) }\end{array}$ & $\begin{array}{c}\text { (5) } \\
\text { Gelsolin } \\
\text { Protein NEL } \\
\text { Argininosuccinate synthase } \\
\text { Fibrinogen beta chain } \\
\text { Apolipoprotein A-I }\end{array}$ & $\begin{array}{c}\text { (3) } \\
\text { Gelsolin } \\
\text { Astacin-like } \\
\text { metalloendopeptidase } \\
\text { Apolipoprotein A-I }\end{array}$ & $\begin{array}{c}\text { (8) } \\
\text { Gelsolin } \\
\text { Protein NEL } \\
\text { Astacin-like metalloendopeptidase } \\
\text { Fibrinogen alpha chain } \\
\text { Thioredoxin } \\
\text { Fibrinogen beta chain } \\
\text { Src substrate protein p85 } \\
\text { Apolipoprotein A-I }\end{array}$ \\
\hline 3 & $\begin{array}{l}\text { Localization } \\
\text { (GO:0051179) }\end{array}$ & $\begin{array}{c}\text { (4) } \\
\text { Serum albumin } \\
\text { Retinol-binding protein } 4 \\
\text { Transthyretin } \\
\text { Apolipoprotein A-I }\end{array}$ & $\begin{array}{c}\text { (5) } \\
\text { Serum albumin } \\
\text { Astacin-like } \\
\text { metalloendopeptidase } \\
\text { Retinol-binding protein } 4 \\
\text { Transthyretin } \\
\text { Apolipoprotein A-I }\end{array}$ & $\begin{array}{c}\text { (7) } \\
\text { Hemoglobin subunit alpha-D } \\
\text { Serum albumin } \\
\text { Astacin-like metalloendopeptidase } \\
\text { Retinol-binding protein } 4 \\
\text { Coagulation factor IX } \\
\text { Transthyretin } \\
\text { Apolipoprotein A-I } \\
\end{array}$ \\
\hline 4 & $\begin{array}{l}\text { Apoptotic process } \\
\text { (GO:0006915) }\end{array}$ & $\begin{array}{c}\text { (1) } \\
\text { Trypsin inhibitor ClTI-1 }\end{array}$ & $\begin{array}{c}\text { (1) } \\
\text { Trypsin inhibitor ClTI-1 }\end{array}$ & $\begin{array}{c}(2) \\
\text { Coagulation factor IX } \\
\text { Trypsin inhibitor ClTI-1 }\end{array}$ \\
\hline 5 & $\begin{array}{l}\text { Biological regulation } \\
\text { (GO:0065007) }\end{array}$ & $\begin{array}{c}\text { (1) } \\
\text { Apolipoprotein A-I }\end{array}$ & $\begin{array}{c}\qquad(2) \\
\text { Astacin-like } \\
\text { metalloendopeptidase } \\
\text { Apolipoprotein A-I }\end{array}$ & $\begin{array}{c}\text { (4) } \\
\text { Astacin-like metalloendopeptidase } \\
\text { Coagulation factor IX } \\
\text { Thioredoxin } \\
\text { Apolipoprotein A-I } \\
\end{array}$ \\
\hline 6 & $\begin{array}{l}\text { Response to stimulus } \\
\text { (GO:0050896) }\end{array}$ & $\begin{array}{c}\text { (2) } \\
\text { Gallinacin-9 } \\
\text { Apolipoprotein A-I }\end{array}$ & $\begin{array}{c}\text { (3) } \\
\text { Astacin-like } \\
\text { metalloendopeptidase } \\
\text { Gallinacin-9 } \\
\text { Apolipoprotein A-I }\end{array}$ & $\begin{array}{c}\text { (5) } \\
\text { Astacin-like metalloendopeptidase } \\
\text { Coagulation factor IX } \\
\text { Thioredoxin } \\
\text { Gallinacin-9 } \\
\text { Apolipoprotein A-I } \\
\end{array}$ \\
\hline 7 & $\begin{array}{l}\text { Developmental process } \\
\text { (GO:0032502) }\end{array}$ & $\begin{array}{c}\text { (4) } \\
\text { Fatty acid-binding protein, } \\
\text { brain } \\
\text { Gelsolin } \\
\end{array}$ & $\begin{array}{c}\text { (4) } \\
\text { Fatty acid-binding protein, } \\
\text { brain } \\
\text { Gelsolin } \\
\end{array}$ & $\begin{array}{l}\text { (7) } \\
\text { Fatty acid-binding protein, brain } \\
\text { Gelsolin } \\
\text { Protein NEL }\end{array}$ \\
\hline
\end{tabular}




\begin{tabular}{|c|c|c|c|c|}
\hline & & $\begin{array}{c}\text { Protein NEL } \\
\text { Apolipoprotein A-I }\end{array}$ & $\begin{array}{c}\text { Astacin-like } \\
\text { metalloendopeptidase } \\
\text { Apolipoprotein A-I }\end{array}$ & $\begin{array}{l}\text { Astacin-like metalloendopeptidase } \\
\text { Coagulation factor IX } \\
\text { Src substrate protein p85 } \\
\text { Apolipoprotein A-I } \\
\end{array}$ \\
\hline 8 & $\begin{array}{l}\text { Multicellular organismal } \\
\text { process (GO:0032501) }\end{array}$ & $\begin{array}{c}\text { (1) } \\
\text { Apolipoprotein A-I }\end{array}$ & $\begin{array}{c}\qquad \text { (2) } \\
\text { Astacin-like } \\
\text { metalloendopeptidase } \\
\text { Apolipoprotein A-I }\end{array}$ & $\begin{array}{c}\text { (5) } \\
\text { Hemoglobin subunit alpha-D } \\
\text { Astacin-like metalloendopeptidase } \\
\text { Fibrinogen alpha chain } \\
\text { Coagulation factor IX } \\
\text { Apolipoprotein A-I } \\
\end{array}$ \\
\hline 9 & $\begin{array}{l}\text { Biological adhesion } \\
\text { (GO:0022610) }\end{array}$ & $\begin{array}{l}\text { (2) } \\
\text { Apolipoprotein A-I } \\
\text { Ovotransferrin }\end{array}$ & $\begin{array}{l}\text { (2) } \\
\text { Astacin-like } \\
\text { metalloendopeptidase } \\
\text { Ovotransferrin }\end{array}$ & $\begin{array}{c}\text { (4) } \\
\text { Astacin-like metalloendopeptidase } \\
\text { Fibrinogen alpha chain } \\
\text { Fibrinogen beta chain } \\
\text { Ovotransferrin } \\
\end{array}$ \\
\hline 10 & $\begin{array}{l}\text { Metabolic process } \\
\text { (GO:0008152) }\end{array}$ & $\begin{array}{c}\text { (10) } \\
\text { Annexin A5 } \\
\text { L-lactate dehydrogenase A } \\
\text { chain } \\
\text { Beta-enolase } \\
\text { Malate dehydrogenase, } \\
\text { cytoplasmic } \\
\text { L-lactate dehydrogenase B } \\
\text { chain } \\
\text { Triosephosphate isomerase } \\
\text { Argininosuccinate synthase } \\
\text { Gallinacin-9 } \\
\text { Alpha-enolase } \\
\text { Apolipoprotein A-I }\end{array}$ & $\begin{array}{c}\text { (8) } \\
\text { Malate dehydrogenase, } \\
\text { cytoplasmic } \\
\text { L-lactate dehydrogenase B } \\
\text { chain } \\
\text { Triosephosphate isomerase } \\
\text { Astacin-like } \\
\text { metalloendopeptidase } \\
\text { Gallinacin-9 } \\
\text { Glyceraldehyde-3-phosphate } \\
\text { dehydrogenase } \\
\text { Alpha-enolase } \\
\text { Apolipoprotein A-I }\end{array}$ & $\begin{array}{c}\text { (13) } \\
\text { Beta-enolase } \\
\text { Malate dehydrogenase, cytoplasmic } \\
\text { Phosphoglycerate kinase } \\
\text { L-lactate dehydrogenase B chain } \\
\text { Triosephosphate isomerase } \\
\text { Astacin-like metalloendopeptidase } \\
\text { Gamma-enolase } \\
\text { Coagulation factor IX } \\
\text { Thioredoxin } \\
\text { Gallinacin-9 } \\
\text { Src substrate protein p85 } \\
\text { Alpha-enolase } \\
\text { Apolipoprotein A-I }\end{array}$ \\
\hline 11 & $\begin{array}{l}\text { Immune system process } \\
\text { (GO:0002376) }\end{array}$ & $\begin{array}{c}\text { (3) } \\
\text { Gallinacin-9 } \\
\text { Ig mu chain C region } \\
\text { Ig lambda chain C region }\end{array}$ & $\begin{array}{c}\text { (5) } \\
\text { Astacin-like } \\
\text { metalloendopeptidase } \\
\text { Gallinacin-9 } \\
\text { Complement factor B-like } \\
\text { protease } \\
\text { Ig mu chain C region } \\
\text { Ig lambda chain C region }\end{array}$ & $\begin{array}{c}\text { (6) } \\
\text { Astacin-like metalloendopeptidase } \\
\text { Coagulation factor IX } \\
\text { Gallinacin-9 } \\
\text { Complement factor B-like protease } \\
\text { Ig mu chain C region } \\
\text { Ig lambda chain C region }\end{array}$ \\
\hline 12 & Fertilization (GO:0009566) & $\begin{array}{c}\text { (1) } \\
\text { Trypsin inhibitor ClTI-1 }\end{array}$ & $\begin{array}{c}\text { (1) } \\
\text { Trypsin inhibitor ClTI-1 }\end{array}$ & $\begin{array}{c}\text { (1) } \\
\text { Trypsin inhibitor ClTI-1 }\end{array}$ \\
\hline
\end{tabular}


Table 2B. Protein class analysis of identified proteins in the seminal fluid Red Junglefowl (RJF), White Leghorn (WL) and Advanced Intercross Line (AIL) male chicken.

\begin{tabular}{|c|c|c|c|c|}
\hline \multirow{2}{*}{\multicolumn{2}{|c|}{ Category name (Accession) }} & \multicolumn{3}{|c|}{ (Number of protein identified) Identified protein symbols } \\
\hline & & RJF & WL & AIL \\
\hline 1 & $\begin{array}{l}\text { Extracellular matrix protein } \\
\text { (PC00102) }\end{array}$ & - & $\begin{array}{l}\text { (1) } \\
\text { Astacin-like metalloendopeptidase }\end{array}$ & $\begin{array}{l}\text { (1) } \\
\text { Astacin-like metalloendopeptidase }\end{array}$ \\
\hline 2 & Protease (PC00190) & - & $\begin{array}{l}\text { (2) } \\
\text { Astacin-like metalloendopeptidase } \\
\text { Complement factor B-like protease }\end{array}$ & $\begin{array}{l}\text { (3) } \\
\text { Astacin-like metalloendopeptidase } \\
\text { Coagulation factor IX } \\
\text { Complement factor B-like protease }\end{array}$ \\
\hline 3 & $\begin{array}{l}\text { Cytoskeletal protein } \\
\text { (PC00085) }\end{array}$ & $\begin{array}{c}\text { (1) } \\
\text { Gelsolin }\end{array}$ & $\begin{array}{c}\text { (1) } \\
\text { Gelsolin }\end{array}$ & $\begin{array}{c}\text { (5) } \\
\text { Gelsolin } \\
\text { Src substrate protein p85 } \\
\text { Tubulin alpha-5 chain } \\
\text { Tubulin beta-1 chain } \\
\text { Tubulin beta-5 chain } \\
\end{array}$ \\
\hline 4 & Transporter (PC00227) & $\begin{array}{c}\text { (2) } \\
\text { Transthyretin } \\
\text { Ovotransferrin }\end{array}$ & $\begin{array}{c}\text { (3) } \\
\text { Astacin-like metalloendopeptidase } \\
\text { Transthyretin } \\
\text { Ovotransferrin } \\
\end{array}$ & $\begin{array}{c}\text { (3) } \\
\text { Astacin-like metalloendopeptidase } \\
\text { Transthyretin } \\
\text { Ovotransferrin } \\
\end{array}$ \\
\hline 5 & Transferase (PC00220) & - & - & $\begin{array}{c}\text { (1) } \\
\text { Phosphoglycerate kinase }\end{array}$ \\
\hline 6 & oxidoreductase (PC00176) & $\begin{array}{c}\text { (3) } \\
\text { L-lactate dehydrogenase A } \\
\text { chain } \\
\text { Malate dehydrogenase, } \\
\text { cytoplasmic } \\
\text { L-lactate dehydrogenase B } \\
\text { chain }\end{array}$ & $\begin{array}{l}\text { (4) } \\
\text { Malate dehydrogenase, cytoplasmic } \\
\text { L-lactate dehydrogenase B chain } \\
\text { Astacin-like metalloendopeptidase } \\
\text { Glyceraldehyde-3-phosphate } \\
\text { dehydrogenase }\end{array}$ & $\begin{array}{l}\text { (4) } \\
\text { Malate dehydrogenase, cytoplasmic } \\
\text { L-lactate dehydrogenase B chain } \\
\text { Astacin-like metalloendopeptidase } \\
\text { Thioredoxin }\end{array}$ \\
\hline 7 & Lyase (PC00144) & $\begin{array}{l}\qquad(2) \\
\text { Beta-enolase } \\
\text { Alpha-enolase }\end{array}$ & $\begin{array}{c}\text { (1) } \\
\text { Alpha-enolase }\end{array}$ & $\begin{array}{c}\text { (3) } \\
\text { Beta-enolase } \\
\text { Gamma-enolase } \\
\text { Alpha-enolase }\end{array}$ \\
\hline 8 & $\begin{array}{l}\text { Cell adhesion molecule } \\
\text { (PC00069) }\end{array}$ & - & $\begin{array}{l}\text { (1) } \\
\text { Astacin-like metalloendopeptidase }\end{array}$ & $\begin{array}{l}\text { (1) } \\
\text { Astacin-like metalloendopeptidase }\end{array}$ \\
\hline 9 & $\begin{array}{l}\text { Signaling molecule } \\
\text { (PC00207) }\end{array}$ & $\begin{array}{l}\text { (1) } \\
\text { Fibrinogen beta chain }\end{array}$ & $\begin{array}{l}\text { (1) } \\
\text { Astacin-like metalloendopeptidase }\end{array}$ & $\begin{array}{l}\text { (4) } \\
\text { Astacin-like metalloendopeptidase } \\
\text { Fibrinogen alpha chain } \\
\text { Coagulation factor IX Fibrinogen } \\
\text { beta chain } \\
\end{array}$ \\
\hline 10 & Enzyme modulator & (3) & (4) & (5) \\
\hline
\end{tabular}




\begin{tabular}{|c|c|c|c|c|}
\hline & (PC00095) & $\begin{array}{c}\text { Ovoinhibitor } \\
\text { Cystatin } \\
\text { Trypsin inhibitor ClTI-1 }\end{array}$ & $\begin{array}{l}\text { Astacin-like metalloendopeptidase } \\
\text { Ovoinhibitor } \\
\text { Cystatin } \\
\text { Trypsin inhibitor CITI-1 }\end{array}$ & $\begin{array}{c}\text { Astacin-like metalloendopeptidase } \\
\text { Coagulation factor IX } \\
\text { Ovoinhibitor } \\
\text { Cystatin } \\
\text { Trypsin inhibitor ClTI-1 } \\
\end{array}$ \\
\hline 11 & $\begin{array}{l}\text { Calcium-binding protein } \\
\text { (PC00060) }\end{array}$ & $\begin{array}{c}\text { (2) } \\
\text { Gelsolin } \\
\text { Protein NEL }\end{array}$ & $\begin{array}{c}(1) \\
\text { Gelsolin }\end{array}$ & $\begin{array}{c}\text { (3) } \\
\text { Gelsolin } \\
\text { Protein NEL } \\
\text { Coagulation factor IX }\end{array}$ \\
\hline 12 & $\begin{array}{l}\text { Defense/immunity protein } \\
\text { (PC00090) }\end{array}$ & $\begin{array}{c}\text { (3) } \\
\text { Gallinacin-9 } \\
\text { Ig mu chain C region } \\
\text { Ig lambda chain C region }\end{array}$ & $\begin{array}{c}\text { (4) } \\
\text { Gallinacin-9 } \\
\text { Complement factor B-like protease } \\
\text { Ig mu chain C region } \\
\text { Ig lambda chain C region } \\
\end{array}$ & $\begin{array}{c}\text { (4) } \\
\text { Gallinacin-9 } \\
\text { Complement factor B-like protease } \\
\text { Ig mu chain C region } \\
\text { Ig lambda chain C region }\end{array}$ \\
\hline 13 & Hydrolase (PC00121) & - & $\begin{array}{l}\text { (2) } \\
\text { Astacin-like metalloendopeptidase } \\
\text { Complement factor B-like protease }\end{array}$ & $\begin{array}{l}\text { (3) } \\
\text { Astacin-like metalloendopeptidase } \\
\text { Coagulation factor IX } \\
\text { Complement factor B-like protease }\end{array}$ \\
\hline 14 & $\begin{array}{l}\text { Transfer/carrier protein } \\
\text { (PC00219) }\end{array}$ & $\begin{array}{c}\text { (3) } \\
\text { Serum albumin } \\
\text { Retinol-binding protein } 4 \\
\text { Transthyretin }\end{array}$ & $\begin{array}{c}\text { (4) } \\
\text { Serum albumin } \\
\text { Astacin-like metalloendopeptidase } \\
\text { Retinol-binding protein } 4 \\
\text { Transthyretin }\end{array}$ & $\begin{array}{c}\text { (5) } \\
\text { Hemoglobin subunit alpha-D } \\
\text { Serum albumin } \\
\text { Astacin-like metalloendopeptidase } \\
\text { Retinol-binding protein } 4 \\
\text { Transthyretin } \\
\end{array}$ \\
\hline 15 & $\begin{array}{l}\text { Transcription factor } \\
\text { (PC00218) }\end{array}$ & - & - & $\begin{array}{c}\text { (1) } \\
\text { Src substrate protein p85 }\end{array}$ \\
\hline 16 & Kinase (PC00137) & - & - & $\begin{array}{c}\text { (1) } \\
\text { Phosphoglycerate kinase }\end{array}$ \\
\hline 17 & Isomerase (PC00135) & $\begin{array}{c}\text { (1) } \\
\text { Triosephosphate isomerase }\end{array}$ & $\begin{array}{c}\text { (1) } \\
\text { Triosephosphate isomerase }\end{array}$ & $\begin{array}{c}\text { (1) } \\
\text { Triosephosphate isomerase }\end{array}$ \\
\hline 18 & Receptor (PC00197) & - & $\begin{array}{l}\text { (1) } \\
\text { Astacin-like metalloendopeptidase }\end{array}$ & $\begin{array}{c}\text { (2) } \\
\text { Astacin-like metalloendopeptidase } \\
\text { Coagulation factor IX }\end{array}$ \\
\hline 19 & Ligase (PC00142) & $\begin{array}{l}\text { (1) } \\
\text { Argininosuccinate synthase }\end{array}$ & - & - \\
\hline
\end{tabular}



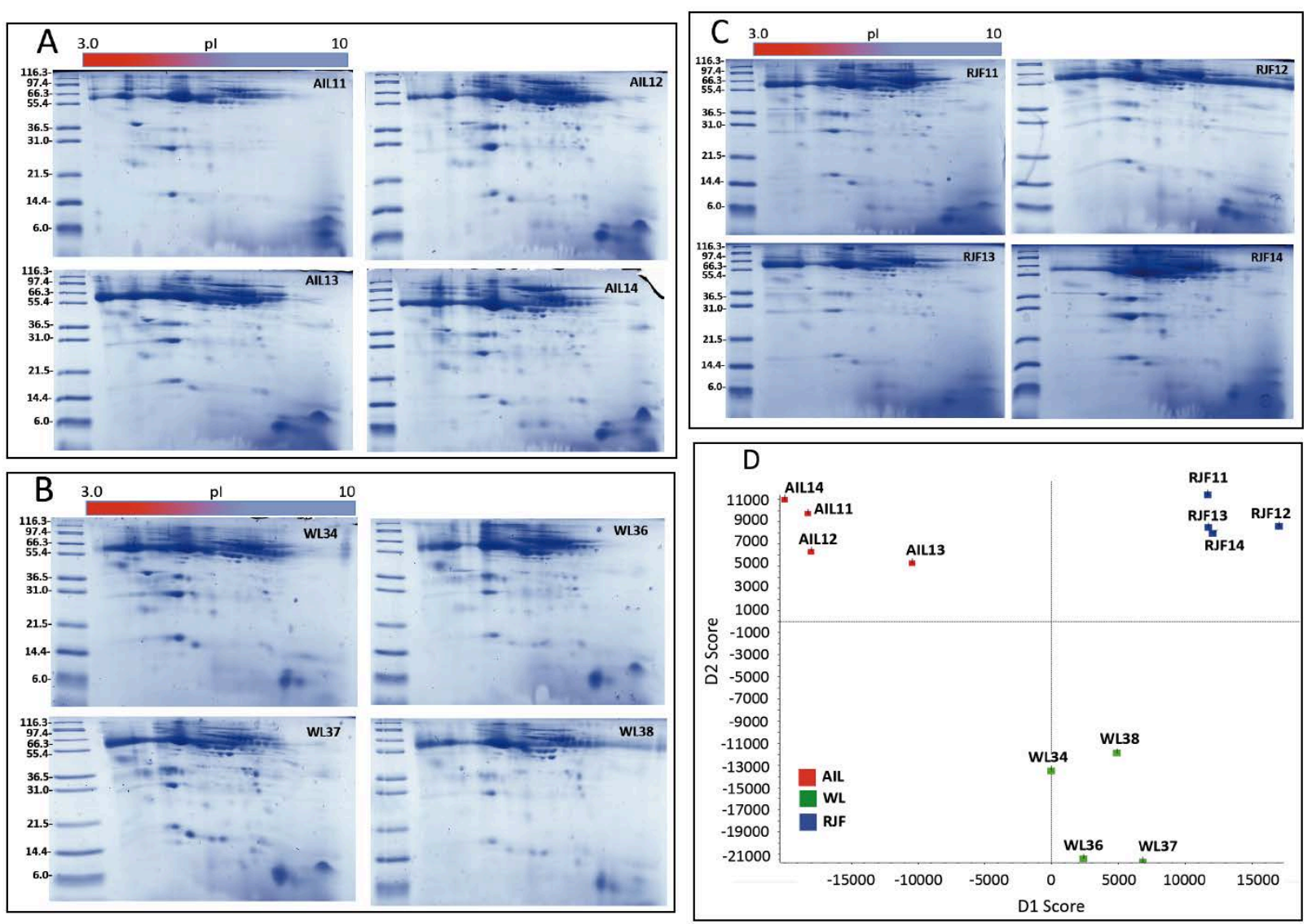

Figure 1. Coomassie Brilliant Blue stained 2D gels of the seminal fluid of individual males $(n=4)$ from each the Advanced Intercross Line (AIL, panel A), White Leghorn (WL, panel B), and Red Junglefowl (RJF, panel C) groups. Panel D presents a PCA plot showing the overall degree of intra- and intergroup variability. 

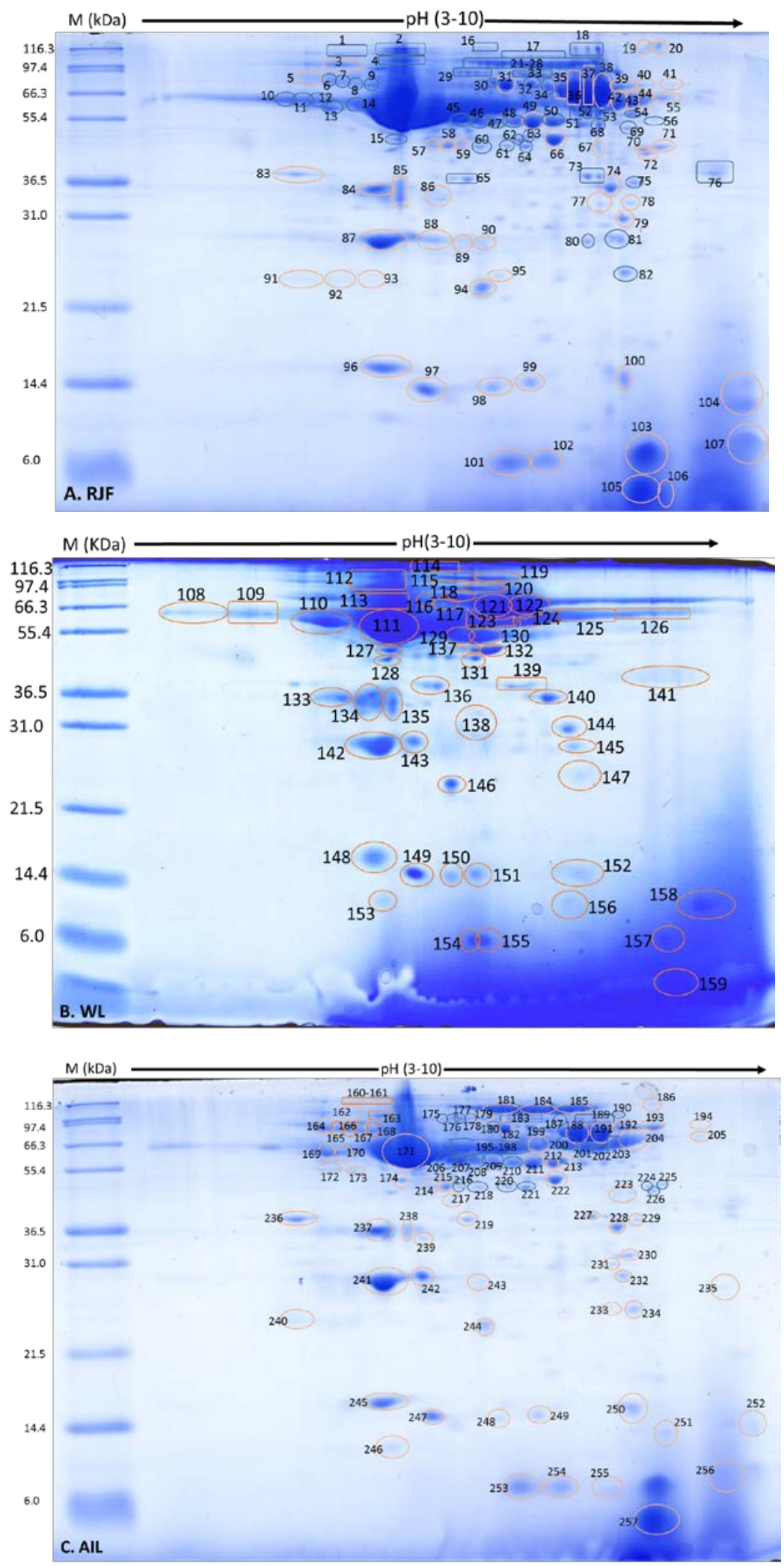

Figure 2. Coomassie Brilliant Blue stained 2D gels of pooled seminal fluid of Red Junglefowl (A, $n=15$ ), White Leghorn $(B, n=15)$ and Advanced Intercross Line $(C, n=15)$ roosters. The gel spots (see the numerical numbers for each spot) were examined by LC-ESI-QTOF-MS/MS followed by Mascot ions search using SwissProt high quality, curated protein database. 


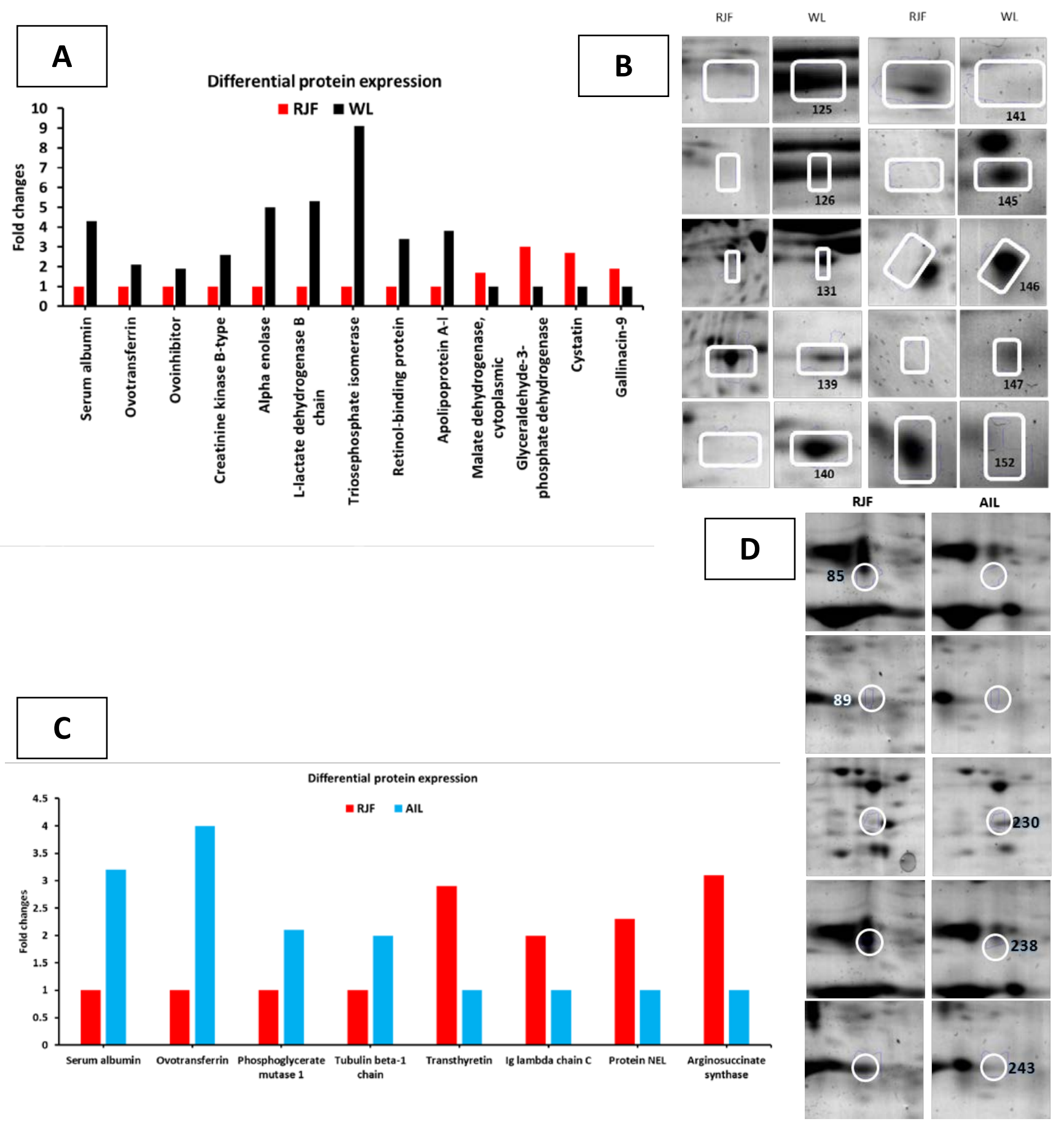

Figure 3. Differentially expressed proteins (based on staining intensities of protein spots on 2D gels) in the seminal fluid (SF) of Red Junglefowl (RJF), White Leghorn (WL) and Advanced Intercross Line (AIL) roosters. A, fold changes of differentially expressed proteins in the SF between RJF (red column) and WL (black column); B, the protein spots on the 2D gels that had different staining intensities as shown in figure A; C, fold changes of differentially expressed proteins in the SF between RJF (red column) and AIL (light blue column); D, the protein spots on the 2D gels that had different staining intensities as shown in figure $\mathrm{C}$. 

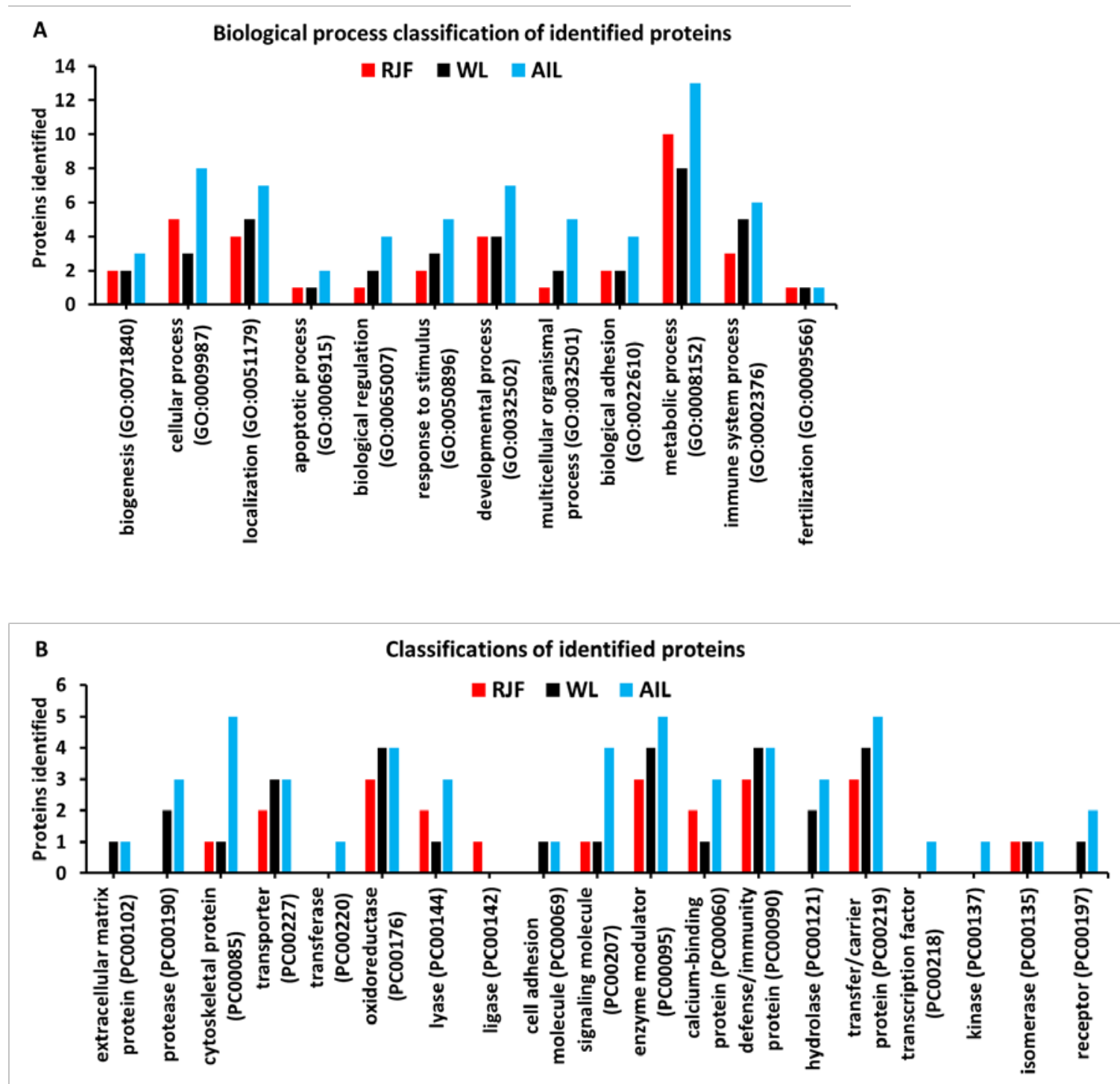

Figure 4. Biological process (A) and protein class (B) analysis of identified proteins in pooled seminal fluid of roosters from the Red Junglefowl $(n=15)$, White Leghorn $(n=15)$ and Advanced Intercross Line $(n=15)$ breeds using PANTHER gene ontology classification following the protocol, described by Mi et al. 2016. 

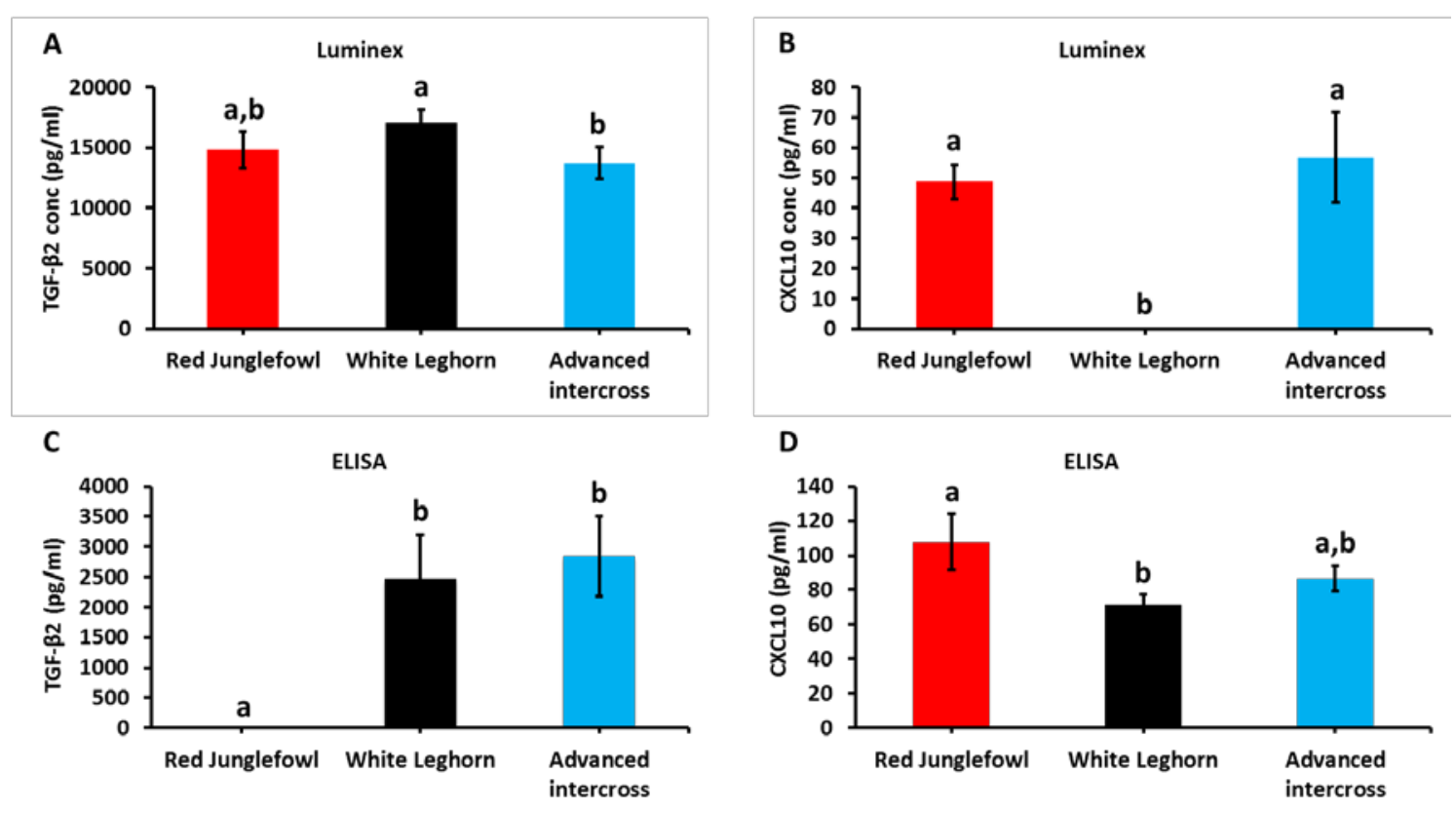

Figure 5. Transforming growth factor $\beta-2$ and CXCL10 concentrations in the seminal fluids of RJF, WL and AIL roosters. In Luminex, individual ejaculates were used to measure the concentrations of TGF- $\beta 2$ (A): RJF (ejaculates, $n=40$ ), WL (ejaculates, $n=79$ ), AIL (ejaculates, $n=39$ ) and CXCL10 (B): RJF (ejaculates, $n=45$ ), WL (ejaculates, $\mathrm{n}=79$ ), AIL (ejaculates, $\mathrm{n}=43$ ). In ELISA, ejaculates of individual birds were pooled and 10 pools (birds, $\mathrm{n}=10$ ) per breed were used to measure the concentrations of TGF- $\beta 2$ (C) and CXCL10 (D). Different letters on the column represents values are significantly different $(\mathrm{P}<0.05)$. 
Identification by LC-MS/MS of the seminal fluid prot

\begin{tabular}{ccccc} 
Spot ID & Breed & Appr. MW (kDa) & Gene Name & Protein Name (Short) \\
\hline $\mathbf{1}$ & RJF & 200 & & NS \\
$\mathbf{2}$ & RJF & 200 & ALB & ALBU_CHICK
\end{tabular}

3 RJF $96 \quad$ NEL $\quad$ NEL_CHICK

4

RJF

156

ALB

ALBU_CHICK

$\begin{array}{lll}\mathbf{5} & \text { RJF } & 77 \\ \mathbf{6} & \text { RJF } & 80 \\ \mathbf{7} & \text { RJF } & 80 \\ & & \\ \mathbf{8} & \text { RJF } & 80 \\ \mathbf{9} & \text { RJF } & 80\end{array}$

77

80

N/A

N/A

ALB

NS

IGHM_CHICK TRFE_CHICK ALBU_CHICK

80

ALB

NS ALBU_CHICK

10

RJF

66.3

ALB

ALBU_CHICK 
11 RJF

12 RJF

13 RJF

14 RJF

60

66.3

MIB2

DDA1

ALB

N/A

16 RJF 200

18 RJF

200

200

19

20

21

22

\begin{abstract}
RJF
RJF
\end{abstract}

100

100

156

156
ALB

N/A

$\mathrm{N} / \mathrm{A}$
NS

ALBU_CHICK

NS

ALBU_CHICK
MIB2_CHICK

DDA1_CHICK

ALBU_CHICK

TRFE_CHICK
ALB

ALB

ALBU_CHICK

TRFE_CHICK

TRFE_CHICK
NS

NS

NS

NS 


$\begin{array}{lcccc}\mathbf{2 3} & \text { RJF } & 156 & & \text { NS } \\ \mathbf{2 4} & \text { RJF } & 156 & \text { CNTN2 } & \text { NS } \\ \mathbf{2 5} & \text { RJF } & 156 & \text { N/A } & \text { CNTN2_CHICK } \\ \mathbf{2 6} & \text { RJF } & 156 & \text { TRFECHICK } \\ & & & \text { N/A } & \text { TRFE_CHICK } \\ \mathbf{2 7} & \text { RJF } & 156 & \text { CNTN2 } & \text { CNTN2_CHICK } \\ & & & \text { N/A } & \text { TRFE_CHICK } \\ \mathbf{2 8} & \text { RJF } & 156 & & \text { NS } \\ \mathbf{2 9} & \text { RJF } & 97.4 & \text { GSN } & \text { GELS_CHICK } \\ \mathbf{3 0} & \text { RJF } & 80 & & \end{array}$


33 RJF

34

RJF

97.4

80

N/A

GSN

66.3

N/A

TRFE_CHICK

35

RJF

66.

36

RJF

66.3

N/A

TRFE CHICK 


\begin{tabular}{|c|c|c|c|c|}
\hline 40 & RJF & 66.3 & & NS \\
\hline 41 & RJF & 66.3 & N/A & TRFE_CHICK \\
\hline 42 & RJF & 60 & ALB & ALBU_CHICK \\
\hline 43 & RJF & 60 & ALB & ALBU_CHICK \\
\hline 44 & RJF & 60 & ALB & ALBU_CHICK \\
\hline 45 & RJF & 55.4 & $\mathrm{OlH}$ & IOV7_CHICK \\
\hline 46 & RJF & 55.4 & $\mathrm{OIH}$ & IOV7_CHICK \\
\hline 47 & RJF & 55.4 & $\mathrm{OIH}$ & IOV7_CHICK \\
\hline 48 & RJF & 55.4 & $\mathrm{OlH}$ & IOV7_CHICK \\
\hline 49 & RJF & 52 & & NS \\
\hline 50 & RJF & 55.4 & FGB & FIBB_CHICK \\
\hline 51 & RJF & 60 & ALB & ALBU_CHICK \\
\hline
\end{tabular}


KPYM_CHICK

FIBB_CHICK 
ENOA_CHICK

ALB

ALBU_CHICK

59

RJF

60

61

62

RJF

RJF

RJF

48

45

48

ASS1

CKB

CKB

$\begin{array}{llc}63 & \text { RJF } & 50 \\ 64 & \text { RJF } & 45 \\ 65 & \text { RJF } & 36.5 \\ 66 & \text { RJF } & 50\end{array}$

GAL10

CDCA8

ENO1
KCRB_CHICK

NS

KCRB_CHICK

ASSY_CHICK

ALBU_CHICK

KCRB_CHICK

GLL10_CHICK
NS
BORE1_CHICK
ENOA_CHICK 


$\begin{array}{llccc} & & & \text { ENO3 } & \text { ENOB_CHICK } \\ \mathbf{6 7} & \text { RJF } & 41 & & \\ \mathbf{6 8} & \text { RJF } & 55.4 & & \text { NS } \\ \mathbf{6 9} & \text { RJF } & 50 & & \text { NS } \\ \mathbf{7 0} & \text { RJF } & 45 & \text { PGK } & \text { PGK_CHICK } \\ \mathbf{7 1} & \text { RJF } & 45 & \text { PGK } & \text { PGK_CHICK }\end{array}$

AATC_CHICK

GOT1

AATC_CHICK

73

RJF

40

MDH1

MDHC_CHICK

74 RJF

36.5

LDHB

LDHB_CHICK

75

RJF

36.5

LDHA

LDHA CHICK

76

77

40

31

78 RJF

31

79 RJF

29

MYOD1

MYOD1_CHICK

NS

NS

TPI1

TPIS_CHICK 
80

$$
\text { RJF }
$$

28

81

RJF

28

82

83

84

RJF

RJF

RJF

23

36.5

36.5

85

86

RJF

RJF

36.5

28

87

RJF

29

88

RJF
N/A

APOA1
APOA1

ALB

GSTM2

APOA1

TPI1

$\begin{array}{cc} & \text { NS } \\ & \text { NS } \\ \text { TTR } & \text { TTHY_CHICK }\end{array}$

TTR

ANXA5

APOA1_CHICK

ALBU_CHICK

TTHY_CHICK

ANXA5_CHICK

APOA1

APOA1 CHICK
LAC_CHICK

APOA1_CHICK 
LAC_CHICK

ALB

ALBU_CHICK

90

RJF

28

N/A

LAC_CHICK

N/A

LAC_CHICK

APOA1

APOA1_CHICK

91 RJF

23

26

VMO1

ALB

VMO1_CHICK

92 RJF

ALBU_CHICK

93

RJF

26

ALB

ALBU_CHICK

94

RJF

25

RBP4

RET4_CHICK

95

RJF

23

APOA1

APOA1_CHICK

NS

96 RJF 15

TTR

TTHY CHICK

97

RJF

14.5

FABP7

FABP7_CHICK 
$\begin{array}{cc}99 & \text { RJF } \\ 100 & \text { RJF }\end{array}$

14

10

101

RJF

6

102

RJF

6

104 RJF

105 RJF

106

107

108

109

110

111

RJF

RJF

RJF

PWL

PWL

PWL

PWL
5

5

4

4

4

66.3

66.3

66

66
N/A

N/A

N/A

GAL9

GAL10

GAL10

N/A

NS

CYT_CHICK

ISK1L_CHICK

ISK1L_CHICK

GAL10

GAL9

GAL10

ALB

ALB

ALB
TRFE_CHICK

GLL9_CHICK

GLL10_CHICK

GLL10_CHICK

GLL10_CHICK

GLL9_CHICK

GLL10 CHICK

NS

ALBU_CHICK

ALBU_CHICK

NS

ALBU_CHICK 
112

PWL

97

N/A

$\mathrm{N} / \mathrm{A}$

ALB
ALBU_CHICK

TRFE_CHICK

IGHM_CHICK

ALBU_CHICK 
PWL

70

N/A

NS

TRFE_CHICK

N/A

ALB

117

PWL

66.3

ALBU_CHICK

TRFE_CHICK 
GELS_CHICK

TRFE_CHICK

120

PWL

95

N/A

TRFE_CHICK

121

PWL

79

$\mathrm{N} / \mathrm{A}$

CFBL_CHICK

N/A

TRFE_CHICK

122

PWL

79

N/A

TRFE_CHICK

123

PWL

66.3

N/A

TRFE CHICK 


$\begin{array}{lllll}124 & \text { PWL } & 66.3 & \text { ALB } & \text { ALBU_CHICK } \\ 125 & \text { PWL } & 66.3 & \text { ALB } & \text { ALBU_CHICK } \\ & & \text { ALB } & \text { ALBU_CHICK }\end{array}$

126

PWL

66.3

ALB

ALBU_CHICK

127

PWL

52

NS

128

PWL

GSN

ALB

129

PWL

55

$\mathrm{OIH}$

130 PWL

55

131 PWL

50

CKB

GELS_CHICK

ALBU_CHICK

IOV7_CHICK

NS

KCRB_CHICK

132

PWL

50

ASS1

ASSY_CHICK

ENO1

ENOA_CHICK 
$133 \quad$ PWL $\quad 36.5$

$134 \quad$ PWL $\quad 36.5$

135

136

137

138

139

140

PWL

PWL

PWL

PWL

PWL

PWL
141

PWL

40

142

PWL

30

GAPDH

APOA1
$\mathrm{MDH} 1$

LDHB

LDHB
NS

TTHY_CHICK
NS

NS

NS

NS

MDHC_CHICK

LDHB_CHICK

LDHB_CHICK
ASTL

ASTL_CHICK

G3P_CHICK

APOA1_CHICK
143

PWL

30

144 PWL

145 PWL

146

PWL

31

28

N/A

LAC_CHICK

APOA1

APOA1_CHICK

TPI1

NS

TPIS_CHICK

RBP4

RET4_CHICK 
147

148

149
PWL

PWL

PWL

14.4

FABP7

APOA1

TTR

15

4

FABP7_CHICK

APOA1_CHICK

$$
\text { TTHY_CHICK }
$$

150

151

152

153

154

PWL

PWL

155

PWL

156

157

158

159

160

161

162

AIL

PWL

PWL

PWL

PWL

AIL

AIL

163

164

165

166

167

168

169
AIL

AIL

AIL

AIL

AIL

AIL

AIL
115

14.4

14.4

14.4

10

6

6

10

6

10

4

200

200

136

90

90

90

90

90

66.3
NS
SK1L_CHICK

ISK1L_CHICK

NR
GLL9_CHICK
NS
NS
NS
ACE_CHICK
NEL_CHICK
NEL_CHICK

F9

FA9_CHICK 
ALBU_CHICK

171

AIL

66.3

NEL

NEL_CHICK

SERPINC1

ANT3_CHICK

ALB

ALBU_CHICK 
N/A TBA5_CHICK

$173 \quad$ AlL

55.4

N/A

TBA5_CHICK

N/A

TBB5_CHICK

$174 \quad$ AIL

48

117

117

117

117

97.4

90

AIL

AIL

AIL

AIL

GSN

181

AIL

150

N/A

ALB

182

AIL

80
NS

NS

NS

NS

NS

NS
GELS_CHICK
TRFE_CHICK

ALBU_CHICK

GELS_CHICK 


$\begin{array}{lcccc} & & & \text { ALB } & \text { ALBU_CHICK } \\ & & & & \\ 183 & \text { AIL } & 117 & \text { N/A } & \text { IGHM_CHICK } \\ 184 & \text { AIL } & 150 & \text { N/A } & \text { CFBL_CHICK } \\ & & & & \\ 185 & \text { AIL } & 150 & \text { N/A } & \text { TRFE_CHICK } \\ & & & & \text { NS } \\ 186 & \text { AIL } & 150 & & \text { NS } \\ 187 & \text { AIL } & 117 & \text { N/A } & \text { TRFE_CHICK }\end{array}$

189

190

191
AIL

117

AIL

AIL

120

70
ALB

N/A

N/A

N/A
ALBU CHICK

IGHM_CHICK

TRFE_CHICK

NS TRFE_CHICK 
192

AIL

193

AIL

95

194

AIL

68

N/A

GAL9

N/A

GLL9_CHICK

TRFE_CHICK

TRFE_CHICK

195

AIL

66.3

ALB

ALBU_CHICK 
ALBU_CHICK

198

199

AIL

AIL

66.3

64

ALB

$\begin{array}{ll}\text { AIL } & 67 \\ \text { AIL } & 65\end{array}$

$\begin{array}{ll}\text { AIL } & 67 \\ \text { AIL } & 65\end{array}$

201 AIL

202

AIL

203

AIL

70

65

204

AIL

66.3
ALB

ALB

ALB

N/A

N/A

PKM

KPYM_CHICK
ALBU_CHICK

ALBU_CHICK

ALBU_CHICK

TRFE_CHICK

NS

ALBU_CHICK

NS

TRFE_CHICK 
205

206
66.3

60

60

60

208

AIL

209

210

211

212

AIL

213

214

215

216

217
AIL

55

AIL

AIL

AIL

52

55

$\mathrm{OIH}$

NS

IOV7_CHICK

IOV7_CHICK

IOV7_CHICK

IOV7_CHICK

FIBB_CHICK

IOV7_CHICK

FIBB_CHICK

IOV7_CHICK

TTHY_CHICK

FIBB_CHICK

ALB

ALBU_CHICK

FGB

FIBB_CHICK

49

49

48

50

ALB

FGA

ALB

AIL

AIL

AIL

ALB

ALBU_CHICK

FIBA_CHICK

ALBU_CHICK

NS

NS

ALBU_CHICK 
219
220
221

222

ENO2

223

224

225

226

227
AIL

50

ENO1

AIL

AIL

AIL

48

48

CKB

NS

KCRB_CHICK

ENOA_CHICK

ENOG_CHICK

ENO3

ENOB_CHICK

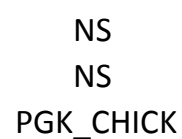

NS

MDH1 MDHC_CHICK 
229

230

AIL

AIL

31.5

27

PGAM1

25.5

231

232

AIL

AIL

25.5

233

234

235

AIL

AIL

AIL

24

24

25

236

237

AIL

39

AIL

35
LDHB

LDHB_CHICK

PGAM1_CHICK

NS

TPI1

TPIS_CHICK 


$\begin{array}{ccccc}238 & \text { AIL } & 35 & \text { TTR } & \text { TTHY_CHICK } \\ & & & & \\ \mathbf{2 3 9} & \text { AIL } & 32 & \text { N/A } & \text { LAC_CHICK } \\ \mathbf{2 4 0} & \text { AIL } & 24 & & \text { NS } \\ \mathbf{2 4 1} & \text { AIL } & 28 & \text { APOA1 } & \text { APOA1_CHICK }\end{array}$

\begin{tabular}{|c|c|c|c|c|}
\hline & & & $\mathrm{N} / \mathrm{A}$ & LAC_CHICK \\
\hline & & & CTTN1 & SRC8_CHICK \\
\hline 242 & AIL & 28 & APOA1 & APOA1_CHICK \\
\hline
\end{tabular}

N/A LAC_CHICK

$243 \quad$ AlL $\quad 28$

N/A

LAC_CHICK

244

AIL

23

RBP4

APOA1

APOA1_CHICK

245

AIL

17

TTR

TTHY_CHICK 
ALBU_CHICK

FABP7

FABP7_CHICK

248

AIL

15

FABP7

N/A

249

AIL

15

FABP7

N/A

FABP7_CHICK

CYT_CHICK

FABP7_CHICK

18

10

N/A

CYT_CHICK

251

AIL

HBAD

N/A

HBAD_CHICK

TRFE_CHICK 
LYZ

ASTL

N/A

AIL

6

253

254

255

256

257
AIL

6

6

AIL

AIL

AIL
6

4
LYSC CHICK

ASTL_CHICK

ISK1L_CHICK

SPT5H_CHICK

ISK1L_CHICK

TRFE_CHICK

NS

GLL9 CHICK

GLL10 CHICK

NS 


\section{Supplementary Table S1}

eins of Red Junglefowl (RJF), White Leghorn (WL) and 9th-generation Advance

Protein name detail

Serum albumin $\mathrm{OS}=$ Gallus gallus $\mathrm{GN}=\mathrm{ALB} P E=1 \mathrm{SV}=2$

Protein NEL OS=Gallus gallus $\mathrm{GN}=\mathrm{NEL} P E=2 \mathrm{SV}=1$

Serum albumin $\mathrm{OS}=$ Gallus gallus $\mathrm{GN}=\mathrm{ALB} P E=1 \mathrm{SV}=2$

Ig mu chain $C$ region $O S=$ Gallus gallus $P E=2 S V=2$

Ovotransferrin $\mathrm{OS}=$ Gallus gallus $\mathrm{PE}=1 \mathrm{SV}=2$

Serum albumin $O S=$ Gallus gallus $G N=A L B P E=1 S V=2$

Serum albumin $\mathrm{OS}=$ Gallus gallus $\mathrm{GN}=\mathrm{ALB} P E=1 \mathrm{SV}=2$

Serum albumin $\mathrm{OS}=$ Gallus gallus $\mathrm{GN}=\mathrm{ALB} P E=1 \mathrm{SV}=2$ 
Serum albumin $\mathrm{OS}=$ Gallus gallus $\mathrm{GN}=\mathrm{ALB} P E=1 \mathrm{SV}=2$

Serum albumin $\mathrm{OS}=$ Gallus gallus $\mathrm{GN}=\mathrm{ALB} P E=1 \mathrm{SV}=2$

E3 ubiquitin-protein ligase MIB2 OS=Gallus gallus $G N=M I B 2 ~ P E=2 ~ S V=1$

DET1- and DDB1-associated protein $1 \mathrm{OS}=$ Gallus gallus $\mathrm{GN}=\mathrm{DDA} 1 \mathrm{PE}=3 \mathrm{SV}=1$

Serum albumin $\mathrm{OS}=$ Gallus gallus $\mathrm{GN}=\mathrm{ALB} P E=1 \mathrm{SV}=2$

Ovotransferrin $\mathrm{OS}=$ Gallus gallus $\mathrm{PE}=1 \mathrm{SV}=2$

Serum albumin $\mathrm{OS}=$ Gallus gallus $\mathrm{GN}=\mathrm{ALB} P E=1 \mathrm{SV}=2$

Ovotransferrin $\mathrm{OS}=$ Gallus gallus $\mathrm{PE}=1 \mathrm{SV}=2$

Ovotransferrin $\mathrm{OS}=$ Gallus gallus $\mathrm{PE}=1 \mathrm{SV}=2$ 
Contactin $-2 \mathrm{OS}=$ Gallus gallus $\mathrm{GN}=\mathrm{CNTN} 2 \mathrm{PE}=1 \mathrm{SV}=1$

Ovotransferrin $\mathrm{OS}=$ Gallus gallus $\mathrm{PE}=1 \mathrm{SV}=2$

Ovotransferrin $\mathrm{OS}=$ Gallus gallus $\mathrm{PE}=1 \mathrm{SV}=2$

Contactin $-2 \mathrm{OS}=$ Gallus gallus $\mathrm{GN}=\mathrm{CNTN} 2 \mathrm{PE}=1 \mathrm{SV}=1$

Ovotransferrin $\mathrm{OS}=$ Gallus gallus $\mathrm{PE}=1 \mathrm{SV}=2$

Gelsolin OS=Gallus gallus GN=GSN PE=2 SV=1

Gelsolin OS=Gallus gallus $\mathrm{GN}=\mathrm{GSN} P E=2 \mathrm{SV}=1$

Ovotransferrin $\mathrm{OS}=$ Gallus gallus $\mathrm{PE}=1 \mathrm{SV}=2$

Ig mu chain $\mathrm{C}$ region $\mathrm{OS}=$ Gallus gallus $\mathrm{PE}=2 \mathrm{SV}=2$

Gelsolin $\mathrm{OS}=$ Gallus gallus $\mathrm{GN}=\mathrm{GSN} P E=2 \mathrm{SV}=1$

Ovotransferrin $\mathrm{OS}=$ Gallus gallus $\mathrm{PE}=1 \mathrm{SV}=2$ 
Ovotransferrin $\mathrm{OS}=$ Gallus gallus $\mathrm{PE}=1 \mathrm{SV}=2$

Gelsolin $\mathrm{OS}=$ Gallus gallus $\mathrm{GN}=\mathrm{GSN} P E=2 \mathrm{SV}=1$

Ovotransferrin $\mathrm{OS}=$ Gallus gallus $\mathrm{PE}=1 \mathrm{SV}=2$

Ovotransferrin $\mathrm{OS}=$ Gallus gallus $\mathrm{PE}=1 \mathrm{SV}=2$ 
Ovotransferrin $\mathrm{OS}=$ Gallus gallus $\mathrm{PE}=1 \mathrm{SV}=2$

Ovotransferrin $\mathrm{OS}=$ Gallus gallus $\mathrm{PE}=1 \mathrm{SV}=2$

Ovotransferrin $\mathrm{OS}=$ Gallus gallus $\mathrm{PE}=1 \mathrm{SV}=2$ 
Ovotransferrin $\mathrm{OS}=$ Gallus gallus $\mathrm{PE}=1 \mathrm{SV}=2$

Serum albumin $\mathrm{OS}=$ Gallus gallus $\mathrm{GN}=\mathrm{ALB} P E=1 \mathrm{SV}=2$

Serum albumin $\mathrm{OS}=$ Gallus gallus $\mathrm{GN}=\mathrm{ALB} P E=1 \mathrm{SV}=2$

Serum albumin $\mathrm{OS}=$ Gallus gallus $\mathrm{GN}=\mathrm{ALB} P E=1 \mathrm{SV}=2$

Ovoinhibitor OS=Gallus gallus $\mathrm{GN}=\mathrm{OIH} \mathrm{PE}=1 \mathrm{SV}=2$

Ovoinhibitor $\mathrm{OS}=$ Gallus gallus $\mathrm{GN}=\mathrm{OIH} \mathrm{PE}=1 \mathrm{SV}=2$

Ovoinhibitor $\mathrm{OS}=$ Gallus gallus $\mathrm{GN}=\mathrm{OIH} \mathrm{PE}=1 \mathrm{SV}=2$

Ovoinhibitor $\mathrm{OS}=$ Gallus gallus $\mathrm{GN}=\mathrm{OIH} \mathrm{PE}=1 \mathrm{SV}=2$

Fibrinogen beta chain (Fragment) $\mathrm{OS}=$ Gallus gallus $\mathrm{GN}=\mathrm{FGB} P \mathrm{PE}=1 \mathrm{SV}=1$

Serum albumin $O S=$ Gallus gallus $G N=A L B P E=1 S V=2$ 
Ovotransferrin $\mathrm{OS}=$ Gallus gallus $\mathrm{PE}=1 \mathrm{SV}=2$

Serum albumin $\mathrm{OS}=$ Gallus gallus $\mathrm{GN}=\mathrm{ALB} P E=1 \mathrm{SV}=2$

Ovotransferrin $\mathrm{OS}=$ Gallus gallus $\mathrm{PE}=1 \mathrm{SV}=2$

Serum albumin $\mathrm{OS}=$ Gallus gallus $\mathrm{GN}=\mathrm{ALB} P E=1 \mathrm{SV}=2$

Pyruvate kinase $\mathrm{PKM}$ OS=Gallus gallus $\mathrm{GN}=\mathrm{PKM} \mathrm{PE}=2 \mathrm{SV}=2$

Serum albumin $\mathrm{OS}=$ Gallus gallus $\mathrm{GN}=\mathrm{ALB} \mathrm{PE}=1 \mathrm{SV}=2$

Pyruvate kinase $P K M$ OS $=$ Gallus gallus $G N=P K M ~ P E=2 S V=2$

Fibrinogen beta chain (Fragment) $\mathrm{OS}=$ Gallus gallus $\mathrm{GN}=\mathrm{FGB} P E=1 \mathrm{SV}=1$

Pyruvate kinase $\mathrm{PKM}$ OS=Gallus gallus $\mathrm{GN}=\mathrm{PKM} P E=2 \mathrm{SV}=2$

Serum albumin $\mathrm{OS}=\mathrm{Gallus}$ gallus $\mathrm{GN}=\mathrm{ALB} P E=1 \mathrm{SV}=2$ 
Alpha-enolase $\mathrm{OS}=\mathrm{G}$ allus gallus $\mathrm{GN}=\mathrm{ENO} 1 \mathrm{PE}=2 \mathrm{SV}=2$

Serum albumin $\mathrm{OS}=$ Gallus gallus $\mathrm{GN}=\mathrm{ALB} P E=1 \mathrm{SV}=2$

Serum albumin $\mathrm{OS}=$ Gallus gallus $\mathrm{GN}=\mathrm{ALB} P E=1 \mathrm{SV}=2$

Creatine kinase $B$-type $O S=$ Gallus gallus $G N=C K B ~ P E=1 ~ S V=1$

Creatine kinase $B$-type $\mathrm{OS}=$ Gallus gallus $\mathrm{GN}=\mathrm{CKB} P E=1 \mathrm{SV}=1$

Argininosuccinate synthase $\mathrm{OS}=\mathrm{G}$ allus gallus $\mathrm{GN}=\mathrm{ASS} 1 \mathrm{PE}=2 \mathrm{SV}=1$

Gallinacin -10 OS=Gallus gallus $\mathrm{GN}=\mathrm{GAL} 10 \mathrm{PE}=2 \mathrm{SV}=1$

Borealin OS=Gallus gallus $\mathrm{GN}=\mathrm{CDCA} 8 \mathrm{PE}=2 \mathrm{SV}=1$

Alpha-enolase $\mathrm{OS}=$ Gallus gallus $\mathrm{GN}=\mathrm{ENO} 1 \mathrm{PE}=2 \mathrm{SV}=2$ 
Beta-enolase $\mathrm{OS}=$ Gallus gallus $\mathrm{GN}=\mathrm{ENO} 3 \mathrm{PE}=1 \mathrm{SV}=3$

Phosphoglycerate kinase $\mathrm{OS}=$ Gallus gallus $\mathrm{GN}=\mathrm{PGK} \mathrm{PE}=2 \mathrm{SV}=2$

Phosphoglycerate kinase $\mathrm{OS}=$ Gallus gallus $\mathrm{GN}=\mathrm{PGK} P E=2 \mathrm{SV}=2$

Aspartate aminotransferase, cytoplasmic $\mathrm{OS}=\mathrm{Gallus}$ gallus $\mathrm{GN}=\mathrm{GOT} 1 \mathrm{PE}=1 \mathrm{SV}=3$

Aspartate aminotransferase, cytoplasmic $\mathrm{OS}=$ Gallus gallus $\mathrm{GN}=\mathrm{GOT} 1 \mathrm{PE}=1 \mathrm{SV}=3$

Malate dehydrogenase, cytoplasmic $\mathrm{OS}=\mathrm{Gallus}$ gallus $\mathrm{GN}=\mathrm{MDH} 1 \mathrm{PE}=2 \mathrm{SV}=1$

L-lactate dehydrogenase $B$ chain $\mathrm{OS}=$ Gallus gallus $\mathrm{GN}=\mathrm{LDHB} P E=1 \mathrm{SV}=3$

L-lactate dehydrogenase $A$ chain $O S=$ Gallus gallus $\mathrm{GN}=\mathrm{LDHA} P E=1 \mathrm{SV}=3$

Myoblast determination protein 1 homolog $\mathrm{OS}=$ Gallus gallus $\mathrm{GN}=\mathrm{MYOD} 1 \mathrm{PE}=2 \mathrm{SV}=2$

Triosephosphate isomerase OS=Gallus gallus $\mathrm{GN}=\mathrm{TPI} 1 \mathrm{PE}=1 \mathrm{SV}=2$ 
Glutathione S-transferase $2 \mathrm{OS}=$ Gallus gallus $\mathrm{GN}=\mathrm{GSTM} 2 \mathrm{PE}=1 \mathrm{SV}=4$

Apolipoprotein A-I OS=Gallus gallus GN=APOA1 PE=1 SV=2

Triosephosphate isomerase $\mathrm{OS}=$ Gallus gallus $\mathrm{GN}=\mathrm{TPI} 1 \mathrm{PE}=1 \mathrm{SV}=2$

Transthyretin OS=Gallus gallus $\mathrm{GN}=\mathrm{TTR} P E=1 \mathrm{SV}=1$

Apolipoprotein A-I OS=Gallus gallus $\mathrm{GN}=\mathrm{APOA} 1 \mathrm{PE}=1 \mathrm{SV}=2$

Serum albumin $\mathrm{OS}=$ Gallus gallus $\mathrm{GN}=\mathrm{ALB} P E=1 \mathrm{SV}=2$

Transthyretin $O S=$ Gallus gallus $G N=T T R P E=1 S V=1$

Annexin $A 5$ OS=Gallus gallus $\mathrm{GN}=\mathrm{ANXA} 5 \mathrm{PE}=1 \mathrm{SV}=2$

Apolipoprotein A-I OS=Gallus gallus $\mathrm{GN}=\mathrm{APOA} 1 \mathrm{PE}=1 \mathrm{SV}=2$

Ig lambda chain $\mathrm{C}$ region $\mathrm{OS}=$ Gallus gallus $\mathrm{PE}=4 \mathrm{SV}=1$

Apolipoprotein A-I OS=Gallus gallus GN=APOA1 PE=1 SV=2 
Ig lambda chain $\mathrm{C}$ region $\mathrm{OS}=$ Gallus gallus $\mathrm{PE}=4 \mathrm{SV}=1$

Serum albumin $\mathrm{OS}=$ Gallus gallus $\mathrm{GN}=\mathrm{ALB} P E=1 \mathrm{SV}=2$

Ig lambda chain $C$ region $O S=$ Gallus gallus $P E=4 S V=1$

Ig lambda chain $C$ region $O S=$ Gallus gallus $P E=4 S V=1$

Apolipoprotein $\mathrm{A}-\mathrm{I} \mathrm{OS}=$ Gallus gallus $\mathrm{GN}=\mathrm{APOA} 1 \mathrm{PE}=1 \mathrm{SV}=2$

Vitelline membrane outer layer protein $1 \mathrm{OS}=\mathrm{Gallus}$ gallus $\mathrm{GN}=\mathrm{VMO} 1 \mathrm{PE}=1 \mathrm{SV}=1$

Serum albumin $\mathrm{OS}=$ Gallus gallus $\mathrm{GN}=\mathrm{ALB} P E=1 \mathrm{SV}=2$

Serum albumin $\mathrm{OS}=$ Gallus gallus $\mathrm{GN}=\mathrm{ALB} P E=1 \mathrm{SV}=2$

Retinol-binding protein $4 \mathrm{OS}=$ Gallus gallus $\mathrm{GN}=\mathrm{RBP} 4 \mathrm{PE}=1 \mathrm{SV}=1$

Apolipoprotein A-I OS=Gallus gallus GN=APOA1 PE=1 SV=2

Transthyretin OS=Gallus gallus $\mathrm{GN}=\mathrm{TTR} P E=1 \mathrm{SV}=1$

Fatty acid-binding protein, brain OS=Gallus gallus $\mathrm{GN}=\mathrm{FABP} 7 \mathrm{PE}=2 \mathrm{SV}=2$ 
Transthyretin $\mathrm{OS}=$ Gallus gallus $\mathrm{GN}=\mathrm{TTR} P E=1 \mathrm{SV}=1$

Fatty acid-binding protein, brain OS=Gallus gallus $\mathrm{GN}=\mathrm{FABP} 7 \mathrm{PE}=2 \mathrm{SV}=2$

Cystatin $\mathrm{OS}=$ Gallus gallus $\mathrm{PE}=1 \mathrm{SV}=2$

Trypsin inhibitor $\mathrm{CITI}-1 \mathrm{OS}=$ Gallus gallus $\mathrm{PE}=1 \mathrm{SV}=1$

Trypsin inhibitor $\mathrm{CITI}-1 \mathrm{OS}=$ Gallus gallus $\mathrm{PE}=1 \mathrm{SV}=1$

Ovotransferrin $\mathrm{OS}=$ Gallus gallus $\mathrm{PE}=1 \mathrm{SV}=2$

Gallinacin -9 OS=Gallus gallus $\mathrm{GN}=\mathrm{GAL} 9 \mathrm{PE}=2 \mathrm{SV}=1$

Gallinacin -10 OS=Gallus gallus $\mathrm{GN}=\mathrm{GAL} 10 \mathrm{PE}=2 \mathrm{SV}=1$

Gallinacin -10 OS=Gallus gallus $\mathrm{GN}=\mathrm{GAL} 10 \mathrm{PE}=2 \mathrm{SV}=1$

Gallinacin -10 OS=Gallus gallus $\mathrm{GN}=\mathrm{GAL} 10 \mathrm{PE}=2 \mathrm{SV}=1$

Gallinacin -9 OS=Gallus gallus $\mathrm{GN}=\mathrm{GAL} 9 \mathrm{PE}=2 \mathrm{SV}=1$

Gallinacin -10 OS=Gallus gallus $\mathrm{GN}=\mathrm{GAL} 10 \mathrm{PE}=2 \mathrm{SV}=1$

Serum albumin $\mathrm{OS}=$ Gallus gallus $\mathrm{GN}=\mathrm{ALB} P E=1 \mathrm{SV}=2$

Serum albumin $\mathrm{OS}=$ Gallus gallus $\mathrm{GN}=\mathrm{ALB} P E=1 \mathrm{SV}=2$

Serum albumin $\mathrm{OS}=$ Gallus gallus $\mathrm{GN}=\mathrm{ALB} P E=1 \mathrm{SV}=2$ 
Serum albumin $\mathrm{OS}=$ Gallus gallus $\mathrm{GN}=\mathrm{ALB} P E=1 \mathrm{SV}=2$

Ovotransferrin $\mathrm{OS}=$ Gallus gallus $\mathrm{PE}=1 \mathrm{SV}=2$

Ig mu chain $C$ region $O S=$ Gallus gallus $P E=2 S V=2$

Serum albumin $\mathrm{OS}=$ Gallus gallus $\mathrm{GN}=\mathrm{ALB} P E=1 \mathrm{SV}=2$ 
Serum albumin $\mathrm{OS}=$ Gallus gallus $\mathrm{GN}=\mathrm{ALB} P E=1 \mathrm{SV}=2$

Ovotransferrin $\mathrm{OS}=$ Gallus gallus $\mathrm{PE}=1 \mathrm{SV}=2$

Ig mu chain $\mathrm{C}$ region $\mathrm{OS}=$ Gallus gallus $\mathrm{PE}=2 \mathrm{SV}=2$

Ovotransferrin $\mathrm{OS}=$ Gallus gallus $\mathrm{PE}=1 \mathrm{SV}=2$

Ig mu chain $\mathrm{C}$ region $\mathrm{OS}=$ Gallus gallus $\mathrm{PE}=2 \mathrm{SV}=2$

Serum albumin $\mathrm{OS}=$ Gallus gallus $\mathrm{GN}=\mathrm{ALB} P E=1 \mathrm{SV}=2$

Ovotransferrin $\mathrm{OS}=$ Gallus gallus $\mathrm{PE}=1 \mathrm{SV}=2$

Ig mu chain $\mathrm{C}$ region $\mathrm{OS}=$ Gallus gallus $\mathrm{PE}=2 \mathrm{SV}=2$

Serum albumin $O S=$ Gallus gallus $G N=A L B P E=1 S V=2$ 
Ovotransferrin $\mathrm{OS}=\mathrm{Gallus}$ gallus $\mathrm{PE}=1 \mathrm{SV}=2$

Gelsolin $\mathrm{OS}=$ Gallus gallus $\mathrm{GN}=\mathrm{GSN} P E=2 \mathrm{SV}=1$

Ovotransferrin $\mathrm{OS}=$ Gallus gallus $\mathrm{PE}=1 \mathrm{SV}=2$

Ovotransferrin $\mathrm{OS}=$ Gallus gallus $\mathrm{PE}=1 \mathrm{SV}=2$

Complement factor $\mathrm{B}$-like protease (Fragment) $\mathrm{OS}=$ Gallus gallus $\mathrm{PE}=1 \mathrm{SV}=1$ Ovotransferrin $\mathrm{OS}=$ Gallus gallus $\mathrm{PE}=1 \mathrm{SV}=2$

Ovotransferrin $\mathrm{OS}=$ Gallus gallus $\mathrm{PE}=1 \mathrm{SV}=2$

Ovotransferrin $\mathrm{OS}=$ Gallus gallus $\mathrm{PE}=1 \mathrm{SV}=2$ 
Serum albumin $\mathrm{OS}=$ Gallus gallus $\mathrm{GN}=\mathrm{ALB} P E=1 \mathrm{SV}=2$

Serum albumin $\mathrm{OS}=$ Gallus gallus $\mathrm{GN}=\mathrm{ALB} P E=1 \mathrm{SV}=2$

Serum albumin $\mathrm{OS}=$ Gallus gallus $\mathrm{GN}=\mathrm{ALB} P E=1 \mathrm{SV}=2$

Serum albumin $\mathrm{OS}=$ Gallus gallus $\mathrm{GN}=\mathrm{ALB} P E=1 \mathrm{SV}=2$

Gelsolin $\mathrm{OS}=$ Gallus gallus $\mathrm{GN}=\mathrm{GSN} P E=2 \mathrm{SV}=1$

Serum albumin $O S=$ Gallus gallus $G N=A L B P E=1 S V=2$

Ovoinhibitor $\mathrm{OS}=$ Gallus gallus $\mathrm{GN}=\mathrm{OIH} \mathrm{PE}=1 \mathrm{SV}=2$

Creatine kinase B-type $\mathrm{OS}=$ Gallus gallus $\mathrm{GN}=\mathrm{CKB} P E=1 \mathrm{SV}=1$

Argininosuccinate synthase $\mathrm{OS}=$ Gallus gallus $\mathrm{GN}=\mathrm{ASS} 1 \mathrm{PE}=2 \mathrm{SV}=1$

Alpha-enolase $\mathrm{OS}=$ Gallus gallus $\mathrm{GN}=\mathrm{ENO} 1 \mathrm{PE}=2 \mathrm{SV}=2$

Beta-enolase $\mathrm{OS}=$ Gallus gallus $\mathrm{GN}=\mathrm{ENO} 3 \mathrm{PE}=1 \mathrm{SV}=3$ 
Transthyretin OS=Gallus gallus $\mathrm{GN}=\mathrm{TTR} P \mathrm{PE}=1 \mathrm{SV}=1$

Malate dehydrogenase, cytoplasmic $\mathrm{OS}=$ Gallus gallus $\mathrm{GN}=\mathrm{MDH} 1 \mathrm{PE}=2 \mathrm{SV}=1$ L-lactate dehydrogenase $B$ chain $O S=G$ allus gallus $G N=L D H B \quad P E=1 S V=3$ $\mathrm{L}$-lactate dehydrogenase $\mathrm{B}$ chain $\mathrm{OS}=\mathrm{G}$ allus gallus $\mathrm{GN}=\mathrm{LDHB} P E=1 \mathrm{SV}=3$

Astacin-like metalloendopeptidase OS=Gallus gallus $\mathrm{GN}=\mathrm{ASTL} P E=2 \mathrm{SV}=1$

Glyceraldehyde-3-phosphate dehydrogenase $\mathrm{OS}=$ Gallus gallus $\mathrm{GN}=\mathrm{GAPDH} P E=2 \mathrm{SV}=3$ Apolipoprotein A-I OS=Gallus gallus GN=APOA1 $P E=1 \mathrm{SV}=2$

Ig lambda chain $\mathrm{C}$ region $\mathrm{OS}=$ Gallus gallus $\mathrm{PE}=4 \mathrm{SV}=1$

Apolipoprotein $\mathrm{A}-\mathrm{I} \mathrm{OS}=\mathrm{Gallus}$ gallus $\mathrm{GN}=\mathrm{APOA} 1 \mathrm{PE}=1 \mathrm{SV}=2$

Triosephosphate isomerase $\mathrm{OS}=$ Gallus gallus $\mathrm{GN}=\mathrm{TPI} 1 \mathrm{PE}=1 \mathrm{SV}=2$

Retinol-binding protein $4 \mathrm{OS}=$ Gallus gallus $\mathrm{GN}=\mathrm{RBP} 4 \mathrm{PE}=1 \mathrm{SV}=1$ 
Apolipoprotein A-I OS=Gallus gallus GN=APOA1 $\mathrm{PE}=1 \mathrm{SV}=2$

Transthyretin $\mathrm{OS}=$ Gallus gallus $\mathrm{GN}=\mathrm{TTR} \mathrm{PE}=1 \mathrm{SV}=1$

Fatty acid-binding protein, brain OS=Gallus gallus $\mathrm{GN}=\mathrm{FABP7} \mathrm{PE}=2 \mathrm{SV}=2$

Cystatin $\mathrm{OS}=$ Gallus gallus $\mathrm{PE}=1 \mathrm{SV}=2$

Trypsin inhibitor $\mathrm{CITI}-1 \mathrm{OS}=$ Gallus gallus $\mathrm{PE}=1 \mathrm{SV}=1$

Trypsin inhibitor $\mathrm{CITI}-1 \mathrm{OS}=$ Gallus gallus $\mathrm{PE}=1 \mathrm{SV}=1$

Gallinacin-9 OS=Gallus gallus GN=GAL9 PE=2 SV=1

Angiotensin-converting enzyme (Fragment) $\mathrm{OS}=\mathrm{G}$ allus gallus $\mathrm{GN}=\mathrm{ACE} P E=2 \mathrm{SV}=1$

Protein NEL OS=Gallus gallus GN=NEL $P E=2 \mathrm{SV}=1$

Protein NEL OS=Gallus gallus $\mathrm{GN}=\mathrm{NEL} P E=2 \mathrm{SV}=1$

Cytochrome $b-c 1$ complex subunit Rieske, mitochondrial $\mathrm{OS}=$ Gallus gallus $\mathrm{GN}=\mathrm{UQCRFS} 1 \mathrm{PE}=1 \mathrm{SV}=1$ Serum albumin $O S=$ Gallus gallus $G N=A L B P E=1 S V=2$ 
Serum albumin $\mathrm{OS}=$ Gallus gallus $\mathrm{GN}=\mathrm{ALB} P E=1 \mathrm{SV}=2$

Protein NEL OS=Gallus gallus GN=NEL $P E=2 \mathrm{SV}=1$

Antithrombin-III (Fragment) OS=Gallus gallus GN=SERPINC1 $P E=1 \mathrm{SV}=1$

Serum albumin $\mathrm{OS}=$ Gallus gallus $\mathrm{GN}=\mathrm{ALB} P E=1 \mathrm{SV}=2$

Tubulin beta- 1 chain $\mathrm{OS}=$ Gallus gallus $\mathrm{PE}=2 \mathrm{SV}=1$

Tubulin beta- 5 chain $\mathrm{OS}=$ Gallus gallus $\mathrm{PE}=3 \mathrm{SV}=1$ 
Tubulin alpha- 5 chain $\mathrm{OS}=$ Gallus gallus $\mathrm{PE}=3 \mathrm{SV}=1$

Tubulin alpha- 5 chain $\mathrm{OS}=$ Gallus gallus $P E=3 \mathrm{SV}=1$

Tubulin beta -5 chain $\mathrm{OS}=$ Gallus gallus $P E=3 \mathrm{SV}=1$

Gelsolin $\mathrm{OS}=$ Gallus gallus $\mathrm{GN}=\mathrm{GSN} P E=2 \mathrm{SV}=1$

Ovotransferrin $\mathrm{OS}=$ Gallus gallus $\mathrm{PE}=1 \mathrm{SV}=2$

Serum albumin $\mathrm{OS}=$ Gallus gallus $\mathrm{GN}=\mathrm{ALB} P E=1 \mathrm{SV}=2$ Gelsolin $\mathrm{OS}=$ Gallus gallus $\mathrm{GN}=\mathrm{GSN} P E=2 \mathrm{SV}=1$ 
Serum albumin OS=Gallus gallus $\mathrm{GN}=\mathrm{ALB} P E=1 \mathrm{SV}=2$

Ig mu chain $\mathrm{C}$ region $\mathrm{OS}=$ Gallus gallus $\mathrm{PE}=2 \mathrm{SV}=2$

Complement factor $B$-like protease (Fragment) $\mathrm{OS}=$ Gallus gallus $\mathrm{PE}=1 \mathrm{SV}=1$

Ovotransferrin $\mathrm{OS}=$ Gallus gallus $\mathrm{PE}=1 \mathrm{SV}=2$

Ovotransferrin $\mathrm{OS}=$ Gallus gallus $\mathrm{PE}=1 \mathrm{SV}=2$

Serum albumin $\mathrm{OS}=$ Gallus gallus $\mathrm{GN}=\mathrm{ALB} P E=1 \mathrm{SV}=2$

Ig mu chain $\mathrm{C}$ region $\mathrm{OS}=$ Gallus gallus $\mathrm{PE}=2 \mathrm{SV}=2$

Ovotransferrin $\mathrm{OS}=$ Gallus gallus $\mathrm{PE}=1 \mathrm{SV}=2$

Ovotransferrin $\mathrm{OS}=$ Gallus gallus $\mathrm{PE}=1 \mathrm{SV}=2$ 
Ovotransferrin $\mathrm{OS}=$ Gallus gallus $\mathrm{PE}=1 \mathrm{SV}=2$

Gallinacin- 9 OS=Gallus gallus $\mathrm{GN}=\mathrm{GAL} 9 \mathrm{PE}=2 \mathrm{SV}=1$

Ovotransferrin $\mathrm{OS}=$ Gallus gallus $\mathrm{PE}=1 \mathrm{SV}=2$

Ovotransferrin $\mathrm{OS}=$ Gallus gallus $\mathrm{PE}=1 \mathrm{SV}=2$

Serum albumin $\mathrm{OS}=$ Gallus gallus $\mathrm{GN}=\mathrm{ALB} P E=1 \mathrm{SV}=2$ 
Serum albumin $\mathrm{OS}=$ Gallus gallus $\mathrm{GN}=\mathrm{ALB} P E=1 \mathrm{SV}=2$

Serum albumin $\mathrm{OS}=$ Gallus gallus $\mathrm{GN}=\mathrm{ALB} P E=1 \mathrm{SV}=2$

Serum albumin $\mathrm{OS}=$ Gallus gallus $\mathrm{GN}=\mathrm{ALB} P E=1 \mathrm{SV}=2$

Ovotransferrin $\mathrm{OS}=$ Gallus gallus $\mathrm{PE}=1 \mathrm{SV}=2$

Serum albumin $\mathrm{OS}=$ Gallus gallus $\mathrm{GN}=\mathrm{ALB} P E=1 \mathrm{SV}=2$

Serum albumin $\mathrm{OS}=$ Gallus gallus $\mathrm{GN}=\mathrm{ALB} P E=1 \mathrm{SV}=2$

Serum albumin $\mathrm{OS}=$ Gallus gallus $\mathrm{GN}=\mathrm{ALB} P E=1 \mathrm{SV}=2$

Ovotransferrin $\mathrm{OS}=$ Gallus gallus $\mathrm{PE}=1 \mathrm{SV}=2$

Pyruvate kinase $P K M$ OS=Gallus gallus $G N=P K M \quad P E=2 S V=2$ 
Ovoinhibitor $\mathrm{OS}=$ Gallus gallus $\mathrm{GN}=\mathrm{OIH} \mathrm{PE}=1 \mathrm{SV}=2$

Ovoinhibitor $\mathrm{OS}=$ Gallus gallus $\mathrm{GN}=\mathrm{OIH} \mathrm{PE}=1 \mathrm{SV}=2$

Ovoinhibitor $\mathrm{OS}=$ Gallus gallus $\mathrm{GN}=\mathrm{OIH} \mathrm{PE}=1 \mathrm{SV}=2$

Ovoinhibitor $\mathrm{OS}=$ Gallus gallus $\mathrm{GN}=\mathrm{OIH} \mathrm{PE}=1 \mathrm{SV}=2$

Fibrinogen beta chain (Fragment) $\mathrm{OS}=$ Gallus gallus $\mathrm{GN}=\mathrm{FGB} P \mathrm{PE}=1 \mathrm{SV}=1$

Ovoinhibitor $\mathrm{OS}=$ Gallus gallus $\mathrm{GN}=\mathrm{OIH} \mathrm{PE}=1 \mathrm{SV}=2$

Fibrinogen beta chain (Fragment) $\mathrm{OS}=\mathrm{Gallus}$ gallus $\mathrm{GN}=\mathrm{FGB} P \mathrm{PE}=1 \mathrm{SV}=1$

Ovoinhibitor $\mathrm{OS}=$ Gallus gallus $\mathrm{GN}=\mathrm{OIH} P E=1 \mathrm{SV}=2$

Transthyretin $\mathrm{OS}=$ Gallus gallus $\mathrm{GN}=\mathrm{TTR} P \mathrm{PE}=1 \mathrm{SV}=1$

Fibrinogen beta chain (Fragment) $\mathrm{OS}=$ Gallus gallus $\mathrm{GN}=\mathrm{FGB} P E=1 \mathrm{SV}=1$

Serum albumin $\mathrm{OS}=$ Gallus gallus $\mathrm{GN}=\mathrm{ALB} P E=1 \mathrm{SV}=2$

Fibrinogen beta chain (Fragment) $\mathrm{OS}=$ Gallus gallus $\mathrm{GN}=\mathrm{FGB} P E=1 \mathrm{SV}=1$

Serum albumin $\mathrm{OS}=$ Gallus gallus $\mathrm{GN}=\mathrm{ALB} P E=1 \mathrm{SV}=2$

Fibrinogen alpha chain $O S=$ Gallus gallus $G N=F G A \quad P E=1 \quad S V=4$

Serum albumin $\mathrm{OS}=$ Gallus gallus $\mathrm{GN}=\mathrm{ALB} P E=1 \mathrm{SV}=2$

Serum albumin $\mathrm{OS}=$ Gallus gallus $\mathrm{GN}=\mathrm{ALB} P E=1 \mathrm{SV}=2$

Creatine kinase $B$-type $O S=$ Gallus gallus $G N=C K B P E=1 S V=1$

Creatine kinase $B$-type $O S=$ Gallus gallus $G N=C K B \quad P E=1 S V=1$ 
Creatine kinase $B$-type $O S=$ Gallus gallus $G N=C K B \quad P E=1 S V=1$

Alpha-enolase $\mathrm{OS}=$ Gallus gallus $\mathrm{GN}=\mathrm{ENO} 1 \mathrm{PE}=2 \mathrm{SV}=2$

Gamma-enolase OS=Gallus gallus $\mathrm{GN}=\mathrm{ENO} 2 \mathrm{PE}=2 \mathrm{SV}=1$

Beta-enolase $\mathrm{OS}=$ Gallus gallus $\mathrm{GN}=\mathrm{ENO} 3 \mathrm{PE}=1 \mathrm{SV}=3$

Phosphoglycerate kinase $\mathrm{OS}=$ Gallus gallus $\mathrm{GN}=\mathrm{PGK} \mathrm{PE}=2 \mathrm{SV}=2$

Malate dehydrogenase, cytoplasmic $\mathrm{OS}=$ Gallus gallus $\mathrm{GN}=\mathrm{MDH} 1 \mathrm{PE}=2 \mathrm{SV}=1$ 
L-lactate dehydrogenase $B$ chain $\mathrm{OS}=$ Gallus gallus $\mathrm{GN}=\mathrm{LDHB} P E=1 \mathrm{SV}=3$

L-lactate dehydrogenase $B$ chain $O S=$ Gallus gallus $G N=L D H B ~ P E=1 S V=3$

Phosphoglycerate mutase $1 \mathrm{OS}=$ Gallus gallus $\mathrm{GN}=\mathrm{PGAM} 1 \mathrm{PE}=1 \mathrm{SV}=3$

Triosephosphate isomerase OS=Gallus gallus $\mathrm{GN}=\mathrm{TPI} 1 \mathrm{PE}=1 \mathrm{SV}=2$

Ovotransferrin $\mathrm{OS}=$ Gallus gallus $\mathrm{PE}=1 \mathrm{SV}=2$

Gallinacin- 9 OS=Gallus gallus $\mathrm{GN}=\mathrm{GAL} 9 \mathrm{PE}=2 \mathrm{SV}=1$

$39 \mathrm{~S}$ ribosomal protein $\mathrm{L} 50$, mitochondrial $\mathrm{OS}=$ Gallus gallus $\mathrm{GN}=\mathrm{MRPL} 50 \mathrm{PE}=2 \mathrm{SV}=1$

Transthyretin OS=Gallus gallus $\mathrm{GN}=\mathrm{TTR} P \mathrm{PE}=1 \mathrm{SV}=1$

Apolipoprotein A-I OS=Gallus gallus $\mathrm{GN}=\mathrm{APOA} 1 \mathrm{PE}=1 \mathrm{SV}=2$ 
Transthyretin OS=Gallus gallus $\mathrm{GN}=\mathrm{TTR} P E=1 \mathrm{SV}=1$

Ig lambda chain $\mathrm{C}$ region $\mathrm{OS}=\mathrm{Gallus}$ gallus $\mathrm{PE}=4 \mathrm{SV}=1$

Apolipoprotein A-I OS=Gallus gallus $\mathrm{GN}=\mathrm{APOA} 1 \mathrm{PE}=1 \mathrm{SV}=2$

Ig lambda chain $C$ region $O S=$ Gallus gallus $P E=4 S V=1$

Src substrate protein $\mathrm{p} 85 \mathrm{OS}=$ Gallus gallus $\mathrm{GN}=\mathrm{CTTN} 1 \mathrm{PE}=1 \mathrm{SV}=1$

Apolipoprotein A-I OS=Gallus gallus $\mathrm{GN}=\mathrm{APOA} 1 \mathrm{PE}=1 \mathrm{SV}=2$

Ig lambda chain $\mathrm{C}$ region $\mathrm{OS}=$ Gallus gallus $\mathrm{PE}=4 \mathrm{SV}=1$

Ig lambda chain $C$ region $O S=$ Gallus gallus $P E=4 S V=1$

Retinol-binding protein $4 \mathrm{OS}=$ Gallus gallus $\mathrm{GN}=\mathrm{RBP} 4 \mathrm{PE}=1 \mathrm{SV}=1$

Apolipoprotein A-I OS=Gallus gallus $\mathrm{GN}=\mathrm{APOA} 1 \mathrm{PE}=1 \mathrm{SV}=2$

Transthyretin $\mathrm{OS}=$ Gallus gallus $\mathrm{GN}=\mathrm{TTR} P E=1 \mathrm{SV}=1$ 
Serum albumin $\mathrm{OS}=$ Gallus gallus $\mathrm{GN}=\mathrm{ALB} P E=1 \mathrm{SV}=2$

Thioredoxin $\mathrm{OS}=$ Gallus gallus $\mathrm{GN}=\mathrm{TXN} \mathrm{PE}=3 \mathrm{SV}=2$

Fatty acid-binding protein, brain $\mathrm{OS}=$ Gallus gallus $\mathrm{GN}=\mathrm{FABP} 7 \mathrm{PE}=2 \mathrm{SV}=2$

Fatty acid-binding protein, brain OS=Gallus gallus $\mathrm{GN}=\mathrm{FABP} 7 \mathrm{PE}=2 \mathrm{SV}=2$

Cystatin OS=Gallus gallus $\mathrm{PE}=1 \mathrm{SV}=2$

Fatty acid-binding protein, brain $\mathrm{OS}=$ Gallus gallus $\mathrm{GN}=\mathrm{FABP} 7 \mathrm{PE}=2 \mathrm{SV}=2$

Cystatin $O S=$ Gallus gallus $P E=1$ SV $=2$

Cystatin $\mathrm{OS}=$ Gallus gallus $\mathrm{PE}=1 \mathrm{SV}=2$

Hemoglobin subunit alpha-D OS=Gallus gallus $G N=H B A D \quad P E=1 S V=1$

Ovotransferrin $\mathrm{OS}=$ Gallus gallus $\mathrm{PE}=1 \mathrm{SV}=2$ 
Lysozyme $\mathrm{C}$ OS=Gallus gallus $\mathrm{GN}=\mathrm{LYZ} P E=1 \mathrm{SV}=1$

Astacin-like metalloendopeptidase $\mathrm{OS}=$ Gallus gallus $\mathrm{GN}=\mathrm{ASTL} P E=2 \mathrm{SV}=1$

Trypsin inhibitor $\mathrm{CITI}-1 \mathrm{OS}=$ Gallus gallus $\mathrm{PE}=1 \mathrm{SV}=1$

Transcription elongation factor SPT5 OS=Gallus gallus GN=SUPT5H PE=2 SV=1

Trypsin inhibitor $\mathrm{CITI}-1 \mathrm{OS}=$ Gallus gallus $\mathrm{PE}=1 \mathrm{SV}=1$

Ovotransferrin $\mathrm{OS}=$ Gallus gallus $\mathrm{PE}=1 \mathrm{SV}=2$

Gallinacin -9 OS=Gallus gallus $\mathrm{GN}=\mathrm{GAL} 9 \mathrm{PE}=2 \mathrm{SV}=1$

Gallinacin -10 OS=Gallus gallus $\mathrm{GN}=\mathrm{GAL} 10 \mathrm{PE}=2 \mathrm{SV}=1$ 
:d Interbreed Line (RJFxWL) (AIL) separated by 2DE as in panels A-C of Figure 1

\begin{tabular}{llll} 
PI (Gels) & Protein PI (M) $\quad$ Accession Number $\quad$ Nominal Mass (Mr) $\quad$ Protein Score \\
\hline
\end{tabular}

4.3

5.51

P19121

71868

424

4.3

5.23

Q90827

96096

335

5

5.51

P19121

71868

233

3.9

4.1

$$
6.07
$$

P01875

48827

58

6.85

P02789

79551

71868

41

4.2

5.51

$\mathrm{P} 19121$

79

4.5

4.6

5.51

P19121

71868

207

3.5

5.51

P19121

71868

192 
3.7

4

5.51

P19121

71868

392

4.1

4.5

5.51

P19121

71868

544

$\begin{array}{cccc}6.41 & \text { Q5ZIJ9 } & 108211 & 15 \\ 8.74 & \text { Q5ZK14 } & 11934 & 13 \\ 5.51 & \text { P19121 } & 71868 & 27 \\ 6.85 & \text { P02789 } & 79551 & 204\end{array}$

$5 \quad 5.51$

$6.1 \quad 6.85$

P02789

204

$\begin{array}{ccccc} & 5.51 & P 19121 & 71868 & 61 \\ 7 & 6.85 & P 02789 & 79551 & 49 \\ 7.5 & 6.85 & P 02789 & 79551 & 154\end{array}$

8.2

8.5

6

6.1 
6.2

6.3

6.6

P28685

114086

35

6.8

6.85

P02789

79551

69

7

6.85

P02789

79551

44

P28685

114086

26

7.5

6.48

P02789

79551

68

093510

86120

250

6.3

5.93

093510

86120

867

6.85

P02789

79551

266

6.07

P01875

48827

116

6.8

5.93

093510

86120

246

P02789

79551

86 
7.5

6.85

P02789

79551

1760

7.6

6.85

P02789

79551

863

7.9

6.85

P02789

79551

700 
8.2

8.6

6.85

P02789

79551

61

7.9

5.51

P19121

71868

243

8

5.51

P19121

71868

152

8.2

5.51

P19121

71868

306

5.9

6.16

P10184

54394

202

6.1

6.16

P10184

54394

132

6.2

6.16

P10184

54394

97

6.5

6.16

P10184

54394

36

6.9

7.1

7.18

Q02020

53272

44

P19121

71868

269 
7.29

P00548

58434

Q02020

53272

28

166

$\begin{array}{ll} & 7.29 \\ 5.5 & 5.51\end{array}$




$\begin{array}{ccccc} & 6.17 & \mathrm{P} 51913 & 47617 & 46 \\ 5.6 & 5.51 & \mathrm{P} 19121 & 71868 & 427\end{array}$

$\begin{array}{lllll}5.9 & 5.51 & \mathrm{P} 19121 & 71868 & 178\end{array}$

$\begin{array}{llll}5.93 & P 05122 / \text { Q92061 } & 43129 & 115\end{array}$

6.1

$\begin{array}{lllll}6.5 & 5.93 & P 05122 / Q 92061 & 43129 & 139\end{array}$

6.7

6.10

Q5ZJ23

47330

223

$\begin{array}{ccccc}6.9 & 8.24 & \text { Q6QLQ9 } & 7525 & 166 \\ 6.8 & & & & \\ 6 & 10.36 & \text { P86346 } & 32672 & 36 \\ 7.1 & 6.17 & \text { P51913 } & 47617 & 1205\end{array}$


7.7

7.7

8

$8.1 \quad 8.31$

$8.5 \quad 8.31$

8.22

8.22

8.2

7.5

7.8

6.92

7.07

Q5ZME2

P00337

P51903

P51903

P00504

P00504

46134

218

45087

45087

400

46134

115

8.1

7.75

P00340

36776

116

9

7.7

8

8
5.43

6.71
P00940

26832

557 


$\begin{array}{lllll} & 6.85 & P 20136 & 26047 & 82 \\ 7.5 & 5.58 & \text { P08250 } & 30661 & 109 \\ 8 & 6.71 & \text { P00940 } & 26832 & 297\end{array}$

$5 \quad 5.11$

P27731

16356

49

5.6

5.60

P17153

36290

189

4.9

5.58

P08250

30661

459

$\begin{array}{cc} & 6.10 \\ 5.5 & 5.58\end{array}$




$\begin{array}{rrrrr} & 6.10 & \mathrm{P} 20763 & 11525 & 76 \\ 6 & 5.51 & \mathrm{P} 19121 & 71868 & 759\end{array}$

6.10

P20763

11525

76

$6.1 \quad 6.10$

P20763

11525

149

5.58

P08250

30661

94

4

8.82

P41366

20677

50

4.2

5.51

P19121

71868

212

4.7

5.51

P19121

71868

131

6.1

5.93

P41263

22843

201

5.58

P08250

30661

44

6.3

5.11

P27731

16356

410

5.5

5.61

Q05423

15031

636 


\begin{tabular}{|c|c|c|c|c|}
\hline & 5.11 & P27731 & 16356 & 50 \\
\hline 6.2 & 5.61 & Q05423 & 15031 & 355 \\
\hline \multicolumn{5}{|l|}{7} \\
\hline 8 & 7.60 & P01038 & 15562 & 195 \\
\hline 6.7 & 6.02 & P85000 & 6371 & 208 \\
\hline \multirow[t]{2}{*}{7} & 6.02 & P85000 & 6371 & 189 \\
\hline & 6.85 & P02789 & 79551 & 71 \\
\hline \multirow[t]{2}{*}{8.3} & 8.65 & Q6QLR1 / Q09MS3 & 7616 & 132 \\
\hline & 8.24 & Q6QLQ9 & 7525 & 73 \\
\hline 9.5 & 8.24 & Q6QLQ9 & 7525 & 136 \\
\hline \multirow[t]{2}{*}{8.3} & 8.24 & Q6QLQ9 & 7525 & 100 \\
\hline & 8.65 & Q6QLR1 / Q09MS3 & 7616 & 49 \\
\hline 8.6 & 8.24 & Q6QLQ9 & 7525 & 166 \\
\hline \multicolumn{5}{|l|}{9.5} \\
\hline 3 & 5.51 & P19121 & 71868 & 60 \\
\hline 3.3 & 5.51 & P19121 & 71868 & 63 \\
\hline \multicolumn{5}{|c|}{4.2} \\
\hline 5 & 5.51 & P19121 & 71868 & 2634 \\
\hline
\end{tabular}


5.5

6.85

P02789

79551

481

6.07

5.51

5.9 


$\begin{array}{lllll} & 5.51 & \text { P19121 } & 71868 & 40 \\ 7 & 5.51 & \text { P19121 } & 71868 & 70 \\ 7.5 & & & & 309\end{array}$

5

5

5.93

093510

86120

67

P19121

71868

40

6

6.16

P10184

54394

129

6.5

6.2

5.93

P05122/Q92061

43129

193

6.5

6.1

Q5ZJ23

47330

51

6.17

P51913

47617

436 
6.5

5.1

5.6

6.1

6.1

6.9

6.92

Q5ZME2

36748

54

7.07

P00337/093362/Q548X0

36694

28

7.1

7.07

P00337/093362/Q548X0

36694

645

8.5

6.76

PODJJ2

46929

149

5

8.71 J0356; Q90848; Q90849; Q9892

35909

41

5.58

$\mathrm{P} 08250$

30661

921

6.1

5.3

7.5

7.5

6
P20763

P08250

P00940

P41263
11525

30661

26832

22843
115

111

84

101 
P08250

30661

86

5

5.11

P27731

16356

219

5.5

5.61

Q05423

15031

379

6

6.2

7.6

7.6

P01038

15562

83

5

6.1

6.02

P85000

6371

105

6.2

6.02

P85000

6371

145

7.5

8.7

8.65

Q6QLR1 /Q09MS3

7616

130

9

9

5

5.5

5.40

Q10751

Q90827

Q90827

138474

131

5.23

5.23

96096

55

96096

167

5.5

4.6

4.8

5

5.1

5.2

8.68

Q5ZLR5

29710

27

4.7

5.51

P19121

71868

144

5.95

Q804X6

53083

77 


5.23
10.29
5.7

Q90827

96096

42

$5.7 \quad 5.51$

Q03352

11502

35

P19121

71868

2329 
4.95

P09644

4.95

P09644

P09653

50395

5.6

6.1

6.2

6.3

6.4

6.5

6.7

5.93

093510

86120

6.9

6.85

P02789

P19121

093510
194

239

151

300

75

36

603 


\begin{tabular}{|c|c|c|c|c|}
\hline & 5.51 & P19121 & 71868 & 106 \\
\hline & 6.07 & P01875 & 48827 & 65 \\
\hline \multicolumn{5}{|l|}{7} \\
\hline 7.1 & 6.48 & P81475 & 27719 & 122 \\
\hline 7.7 & 6.85 & P02789 & 79551 & 63 \\
\hline \multicolumn{5}{|l|}{8.5} \\
\hline \multicolumn{5}{|l|}{7.4} \\
\hline 7.6 & 6.85 & P02789 & 79551 & 1779 \\
\hline
\end{tabular}

$\begin{array}{ccccc} & 5.51 & \mathrm{P} 19121 & 71868 & 105 \\ & & & & \\ 7.8 & 6.07 & \mathrm{P} 01875 & 48827 & 80 \\ & 6.85 & \mathrm{P} 02789 & 79551 & 106\end{array}$

8.1

7.9

6.85

P02789

79551

1441 
8.1

6.85

8.65

8.5

6.85

6.85

P02789

9

6.5

5.51

P19121

71868

7616

79551

27

300

P02789

79551

511

935 
5.9

7.3

$\begin{array}{rrrrr} & 5.51 & \mathrm{P} 19121 & 71868 & 87 \\ 7.8 & 5.51 & \mathrm{P} 19121 & 71868 \\ 8.1 & 5.51 & \mathrm{P} 19121 & 71868 & 188\end{array}$


9

6.2

6.16

P10184

54394

387

6.3

6.16

P10184

54394

135

6.5

6.16

P10184

54394

222

6.8

6.16

P10184

54394

61

7
7.18

Q02020

53272

66

P10184

54394

34

Q02020

53272

54394

57

P10184

16356

48

P27731

53272

28

95

7.3

7.18

Q02020

71868

79

7.5

5.51

P19121

53272

352

5.51

5.69

6

5.51

6.1

6.2

6.1

5.51

P19121

P19121

71868

68

71868

417

$\begin{array}{ccccc} & 5.93 & \mathrm{P} 05122 / \mathrm{Q} 92061 & 43129 & 77 \\ 6.5 & 5.93 & \mathrm{P} 05122 / \mathrm{Q} 92061 & 43129 & 128\end{array}$


6.5

6.9

7

5.93

P05122/Q92061

43129

214

7.3

6.17

P51913

47617

844

4.84

O57391

47621

246

7.28

P07322

47566

159

8

8.4

8.6

8.31

P51903

45087

123

8.5

7.8

6.92

Q5ZME2

36748

296 
8.3

7.07

P00337 / 093362 / Q548X0

36694

183

8.2

7.03

Q5ZLN1 / P84174

29051

171

8

8.1

6.71

P00940

26832

342

8

8.2

9.2

6.85

P02789

79551

154

8.65

9.12

Q6QLR1 / Q09MS3

7616

18192

43

Q5ZLC1

24

4.2

5.11

P27731

16356

582

5.58

P08250

30661

264 
5.9

6.10

P20763

11525

75

4.3

5.58

P08250

30661

825

\begin{tabular}{|c|c|c|c|c|}
\hline & 6.10 & P20763 & 11525 & 87 \\
\hline & 5.33 & Q01406 & 63462 & 40 \\
\hline 5.9 & 5.58 & P08250 & 30661 & 47 \\
\hline
\end{tabular}

6.10

6.5

6.5

6.10

5.93

5.58

P08250

P27731

5.3

5.11
11525

106

91

176
87

478 
7.60

P01038

15562

$7.1 \quad 5.61$

Q05423

15031

48

8.2

7.60

P01038

15562

272

8.8

7.60

P01038

15562

171

9.8

7.01

P02001 / Q549K7 / Q78AJ9

15742

49

6.85

P02789

79551

289 
PODJJ2

46929

6.02

P85000

6371

179

$\begin{array}{cc} & 5.00 \\ 7.5 & 6.02 \\ & 6.85\end{array}$

Q5ZI08

120306

38

P85000

6371

90

P02789

79551

39

8

8.65

Q6QLR1 / Q09MS3

7616

199

8.24

Q6QLQ9

7525

92

8.6 


\begin{tabular}{llll} 
Coverage\% Peptide Count (S) Peptide lon Score Peptide $\mathrm{m} / \mathrm{z} \quad$ Peptide charge Z \\
\hline
\end{tabular}

\begin{tabular}{|c|c|c|c|c|}
\hline \multirow[t]{10}{*}{$32(615)$} & 19(11) & 51 & 643.8167 & 2 \\
\hline & & 93 & 756.4079 & 2 \\
\hline & & 68 & 499.2741 & 3 \\
\hline & & 48 & 432.7273 & 2 \\
\hline & & 57 & 874.3719 & 2 \\
\hline & & 47 & 509.6128 & 3 \\
\hline & & 48 & 782.0294 & 3 \\
\hline & & 89 & 779.4150 & 2 \\
\hline & & 63 & 965.4402 & 2 \\
\hline & & 84 & 634.8147 & 2 \\
\hline \multirow[t]{8}{*}{21 (816) } & $14(10)$ & 52 & 527.3798 & 2 \\
\hline & & 48 & 684.4836 & 2 \\
\hline & & 87 & 595.9177 & 2 \\
\hline & & 44 & 818.0701 & 2 \\
\hline & & 56 & 774.2372 & 3 \\
\hline & & 62 & 595.4487 & 2 \\
\hline & & 68 & 692.9443 & 2 \\
\hline & & 62 & 741.0199 & 2 \\
\hline \multirow[t]{8}{*}{$24(615)$} & $15(8)$ & 65 & 643.8157 & 2 \\
\hline & & 45 & 756.4021 & 2 \\
\hline & & 72 & 499.2731 & 3 \\
\hline & & 59 & 597.7707 & 2 \\
\hline & & 54 & 773.8997 & 2 \\
\hline & & 42 & 653.3026 & 2 \\
\hline & & 48 & 562.9749 & 3 \\
\hline & & 50 & 516.7468 & 2 \\
\hline $2(446)$ & $1(1)$ & 58 & 733.4302 & 2 \\
\hline 11(705) & $7(0)$ & 35 & 551.6193 & 3 \\
\hline \multirow[t]{3}{*}{ 13(615) } & $8(3)$ & 61 & 597.7708 & 2 \\
\hline & & 44 & 432.7248 & 2 \\
\hline & & 42 & 516.7426 & 2 \\
\hline \multirow[t]{6}{*}{ 26(615) } & $15(5)$ & 79 & 756.4028 & 2 \\
\hline & & 44 & 499.2776 & 3 \\
\hline & & 41 & 432.7265 & 2 \\
\hline & & 49 & 782.0269 & 3 \\
\hline & & 70 & 965.4410 & 2 \\
\hline & & 48 & 413.7938 & 2 \\
\hline \multirow[t]{2}{*}{ 15(615) } & 10(5) & 60 & 643.8108 & 2 \\
\hline & & 70 & 499.2749 & 3 \\
\hline
\end{tabular}




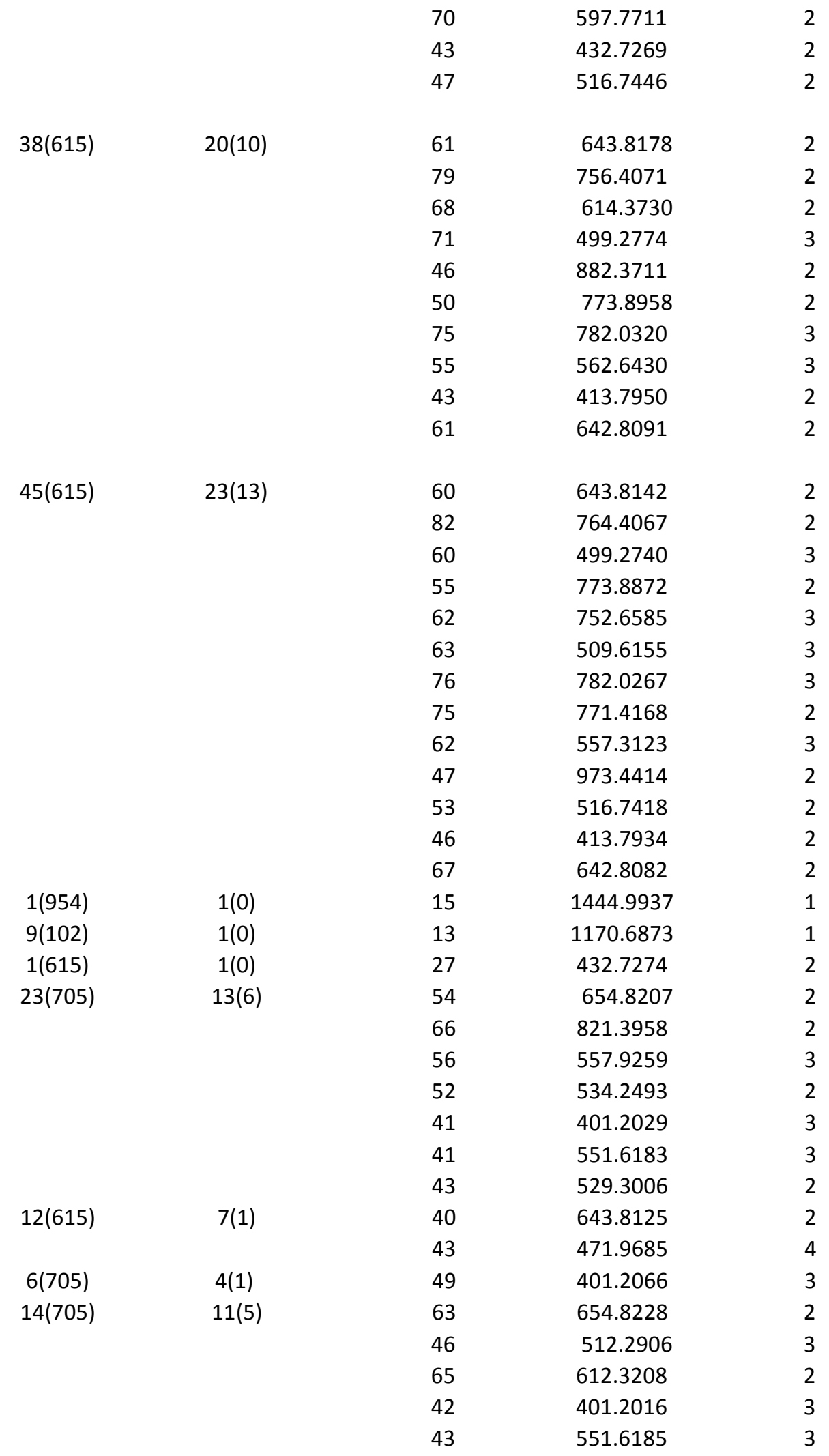




\begin{tabular}{|c|c|c|c|c|}
\hline 1(1036) & $1(0)$ & 35 & 554.6258 & 3 \\
\hline \multirow[t]{2}{*}{ 10(705) } & $6(2)$ & 45 & 512.2842 & 3 \\
\hline & & 53 & 401.2078 & 3 \\
\hline $7(705)$ & $5(1)$ & 44 & 457.2391 & 2 \\
\hline $1(1036)$ & $1(0)$ & 26 & 554.6205 & 3 \\
\hline $11(705)$ & $7(1)$ & 60 & 612.3228 & 2 \\
\hline \multirow[t]{7}{*}{$16(778)$} & 10(7) & 61 & 411.2172 & 2 \\
\hline & & 42 & 639.3550 & 2 \\
\hline & & 55 & 637.3434 & 3 \\
\hline & & 77 & 438.8996 & 3 \\
\hline & & 74 & 752.3856 & 2 \\
\hline & & 64 & 666.8361 & 2 \\
\hline & & 57 & 526.2571 & 2 \\
\hline \multirow[t]{16}{*}{$30(778)$} & $19(16)$ & 59 & 499.7768 & 2 \\
\hline & & 48 & 642.8839 & 2 \\
\hline & & 45 & 457.7734 & 2 \\
\hline & & 152 & 1104.5549 & 2 \\
\hline & & 60 & 639.3556 & 2 \\
\hline & & 67 & 562.2766 & 2 \\
\hline & & 46 & 637.3433 & 3 \\
\hline & & 45 & 673.3321 & 3 \\
\hline & & 82 & 438.8987 & 3 \\
\hline & & 71 & 924.7696 & 3 \\
\hline & & 53 & 573.2326 & 2 \\
\hline & & 82 & 1051.5419 & 2 \\
\hline & & 75 & 610.8287 & 2 \\
\hline & & 90 & 752.3922 & 2 \\
\hline & & 61 & 666.8204 & 2 \\
\hline & & 55 & 526.2555 & 2 \\
\hline \multirow[t]{7}{*}{17 (705) } & $12(7)$ & 58 & 654.8222 & 2 \\
\hline & & 47 & 512.2778 & 3 \\
\hline & & 46 & 447.2706 & 2 \\
\hline & & 61 & 848.4086 & 2 \\
\hline & & 66 & 551.6247 & 3 \\
\hline & & 48 & 666.3378 & 2 \\
\hline & & 49 & 582.9811 & 3 \\
\hline \multirow[t]{2}{*}{$4(446)$} & $2(2)$ & 60 & 498.7280 & 2 \\
\hline & & 89 & 733.4222 & 2 \\
\hline \multirow[t]{7}{*}{ 20(778) } & $12(7)$ & 52 & 499.7821 & 2 \\
\hline & & 52 & 642.8848 & 2 \\
\hline & & 68 & 438.9004 & 3 \\
\hline & & 81 & 924.7762 & 3 \\
\hline & & 58 & 610.8293 & 2 \\
\hline & & 66 & 752.3889 & 2 \\
\hline & & 70 & 526.2550 & 2 \\
\hline \multirow[t]{2}{*}{ 15(705) } & $10(2)$ & 43 & 512.2846 & 3 \\
\hline & & 51 & 534.2554 & 2 \\
\hline
\end{tabular}




\begin{tabular}{|c|c|c|c|c|}
\hline \multirow[t]{11}{*}{$31(705)$} & $21(11)$ & 59 & 479.5845 & 3 \\
\hline & & 61 & 654.8251 & 2 \\
\hline & & 40 & 512.2916 & 3 \\
\hline & & 62 & 821.3993 & 2 \\
\hline & & 48 & 534.2527 & 2 \\
\hline & & 49 & 401.2037 & 3 \\
\hline & & 58 & 551.6154 & 3 \\
\hline & & 88 & 921.9135 & 2 \\
\hline & & 52 & 512.7704 & 4 \\
\hline & & 51 & 529.3013 & 2 \\
\hline & & 86 & 1000.4426 & 2 \\
\hline \multirow[t]{4}{*}{$14(778)$} & 9(4) & 57 & 637.3380 & 3 \\
\hline & & 69 & 438.8925 & 3 \\
\hline & & 62 & 924.7823 & 3 \\
\hline & & 65 & 610.8276 & 2 \\
\hline \multirow[t]{17}{*}{44 (705) } & $27(17)$ & 79 & 718.8795 & 2 \\
\hline & & 78 & 654.8305 & 2 \\
\hline & & 75 & 512.2891 & 3 \\
\hline & & 108 & 980.4918 & 2 \\
\hline & & 92 & 821.3989 & 2 \\
\hline & & 72 & 557.9280 & 3 \\
\hline & & 58 & 447.2747 & 2 \\
\hline & & 83 & 848.4149 & 2 \\
\hline & & 52 & 612.3203 & 2 \\
\hline & & 48 & 534.2622 & 2 \\
\hline & & 47 & 401.2029 & 3 \\
\hline & & 41 & 905.4303 & 3 \\
\hline & & 67 & 551.6181 & 3 \\
\hline & & 89 & 921.9074 & 2 \\
\hline & & 51 & 683.3630 & 3 \\
\hline & & 50 & 529.3034 & 2 \\
\hline & & 77 & 1000.4492 & 2 \\
\hline \multirow[t]{17}{*}{49 (705) } & $30(28)$ & 77 & 942.8324 & 3 \\
\hline & & 55 & 479.5776 & 3 \\
\hline & & 79 & 654.8239 & 2 \\
\hline & & 72 & 512.2844 & 3 \\
\hline & & 103 & 980.4903 & 2 \\
\hline & & 88 & 821.4016 & 2 \\
\hline & & 45 & 518.2783 & 2 \\
\hline & & 62 & 677.3193 & 2 \\
\hline & & 79 & 848.4058 & 2 \\
\hline & & 50 & 612.3137 & 2 \\
\hline & & 51 & 534.2554 & 2 \\
\hline & & 65 & 601.3055 & 2 \\
\hline & & 52 & 443.9002 & 3 \\
\hline & & 54 & 521.2853 & 2 \\
\hline & & 71 & 551.6187 & 3 \\
\hline & & 41 & 602.2862 & 2 \\
\hline & & 113 & 1275.5238 & 2 \\
\hline
\end{tabular}




\begin{tabular}{|c|c|c|c|c|}
\hline & & 47 & 630.3019 & 3 \\
\hline & & 89 & 913.8946 & 2 \\
\hline & & 49 & 512.7651 & 4 \\
\hline & & 42 & 582.9736 & 3 \\
\hline & & 49 & 529.3053 & 2 \\
\hline & & 96 & 1008.4643 & 2 \\
\hline $49(705)$ & $36(27)$ & 131 & 718.8748 & 2 \\
\hline & & 73 & 654.8320 & 2 \\
\hline & & 72 & 512.2889 & 3 \\
\hline & & 89 & 980.4855 & 2 \\
\hline & & 96 & 821.4014 & 2 \\
\hline & & 73 & 557.9329 & 3 \\
\hline & & 66 & 518.2781 & 2 \\
\hline & & 63 & 848.4136 & 2 \\
\hline & & 58 & 612.3239 & 2 \\
\hline & & 51 & 534.7573 & 2 \\
\hline & & 64 & 401.2055 & 3 \\
\hline & & 61 & 443.9089 & 3 \\
\hline & & 46 & 471.2650 & 4 \\
\hline & & 56 & 697.3889 & 2 \\
\hline & & 57 & 521.2880 & 2 \\
\hline & & 60 & 551.6270 & 3 \\
\hline & & 77 & 630.3107 & 3 \\
\hline & & 80 & 913.9117 & 2 \\
\hline & & 51 & 512.7747 & 4 \\
\hline & & 67 & 809.4307 & 3 \\
\hline & & 108 & 873.9764 & 2 \\
\hline & & 50 & 529.3019 & 2 \\
\hline & & 89 & 1000.4610 & 2 \\
\hline 49 (705) & $31(22)$ & 87 & 942.8241 & 3 \\
\hline & & 118 & 718.9291 & 2 \\
\hline & & 78 & 654.8246 & 2 \\
\hline & & 82 & 512.2877 & 3 \\
\hline & & 87 & 980.4872 & 2 \\
\hline & & 80 & 821.3981 & 2 \\
\hline & & 73 & 557.9263 & 3 \\
\hline & & 53 & 447.2696 & 2 \\
\hline & & 102 & 848.4155 & 2 \\
\hline & & 49 & 612.3190 & 2 \\
\hline & & 48 & 534.2531 & 2 \\
\hline & & 58 & 401.2068 & 3 \\
\hline & & 84 & 930.4580 & 3 \\
\hline & & 70 & 551.6205 & 3 \\
\hline & & 41 & 602.2900 & 2 \\
\hline & & 46 & 512.7904 & 4 \\
\hline & & 49 & 582.9820 & 3 \\
\hline & & 50 & 529.3010 & 2 \\
\hline & & 60 & 1000.4441 & 2 \\
\hline 47 (705) & $28(15)$ & 101 & 942.8282 & 3 \\
\hline & & 48 & 479.5895 & 3 \\
\hline
\end{tabular}




\begin{tabular}{|c|c|c|c|c|}
\hline & & 70 & 654.8328 & 2 \\
\hline & & 72 & 512.2890 & 3 \\
\hline & & 80 & 821.3997 & 2 \\
\hline & & 62 & 447.2699 & 2 \\
\hline & & 46 & 848.4064 & 2 \\
\hline & & 55 & 612.3200 & 2 \\
\hline & & 47 & 534.2587 & 2 \\
\hline & & 60 & 601.3044 & 2 \\
\hline & & 40 & 930.8045 & 3 \\
\hline & & 71 & 551.6215 & 3 \\
\hline & & 95 & 1275.5198 & 2 \\
\hline & & 50 & 529.3016 & 2 \\
\hline & & 81 & 1000.4468 & 2 \\
\hline $6(705)$ & $4(1)$ & 54 & 654.9547 & 2 \\
\hline 19 (615) & $10(6)$ & 55 & 643.8194 & 2 \\
\hline & & 85 & 756.4050 & 2 \\
\hline & & 52 & 499.2740 & 3 \\
\hline & & 52 & 771.4164 & 2 \\
\hline & & 70 & 691.8295 & 2 \\
\hline & & 75 & 965.4385 & 2 \\
\hline 24 (615) & $12(5)$ & 47 & 432.7266 & 2 \\
\hline & & 55 & 851.0798 & 3 \\
\hline & & 43 & 782.0326 & 3 \\
\hline & & 62 & 965.4432 & 2 \\
\hline & & 48 & 413.7935 & 2 \\
\hline & & 41 & 616.3156 & 2 \\
\hline 21 (615) & 11(9) & 58 & 643.8179 & 2 \\
\hline & & 95 & 756.4124 & 2 \\
\hline & & 54 & 499.2737 & 3 \\
\hline & & 45 & 597.7664 & 2 \\
\hline & & 49 & 569.8051 & 2 \\
\hline & & 69 & 782.0243 & 3 \\
\hline & & 68 & 771.4116 & 2 \\
\hline & & 67 & 965.4385 & 2 \\
\hline & & 46 & 413.7932 & 2 \\
\hline $18(472)$ & $7(4)$ & 57 & 572.9625 & 3 \\
\hline & & 54 & 568.7460 & 2 \\
\hline & & 62 & 1028.4470 & 3 \\
\hline & & 56 & 603.2683 & 2 \\
\hline $17(472)$ & $7(3)$ & 52 & 578.2848 & 3 \\
\hline & & 53 & 560.7495 & 2 \\
\hline & & 56 & 603.2728 & 2 \\
\hline $12(472)$ & $6(3)$ & 51 & 574.7643 & 2 \\
\hline & & 50 & 568.7442 & 2 \\
\hline & & 49 & 554.2780 & 2 \\
\hline $8(472)$ & $4(0)$ & 36 & 408.7256 & 2 \\
\hline $2(463)$ & $1(1)$ & 44 & 679.3367 & 2 \\
\hline $35(615)$ & $20(9)$ & 81 & 756.4030 & 2 \\
\hline
\end{tabular}




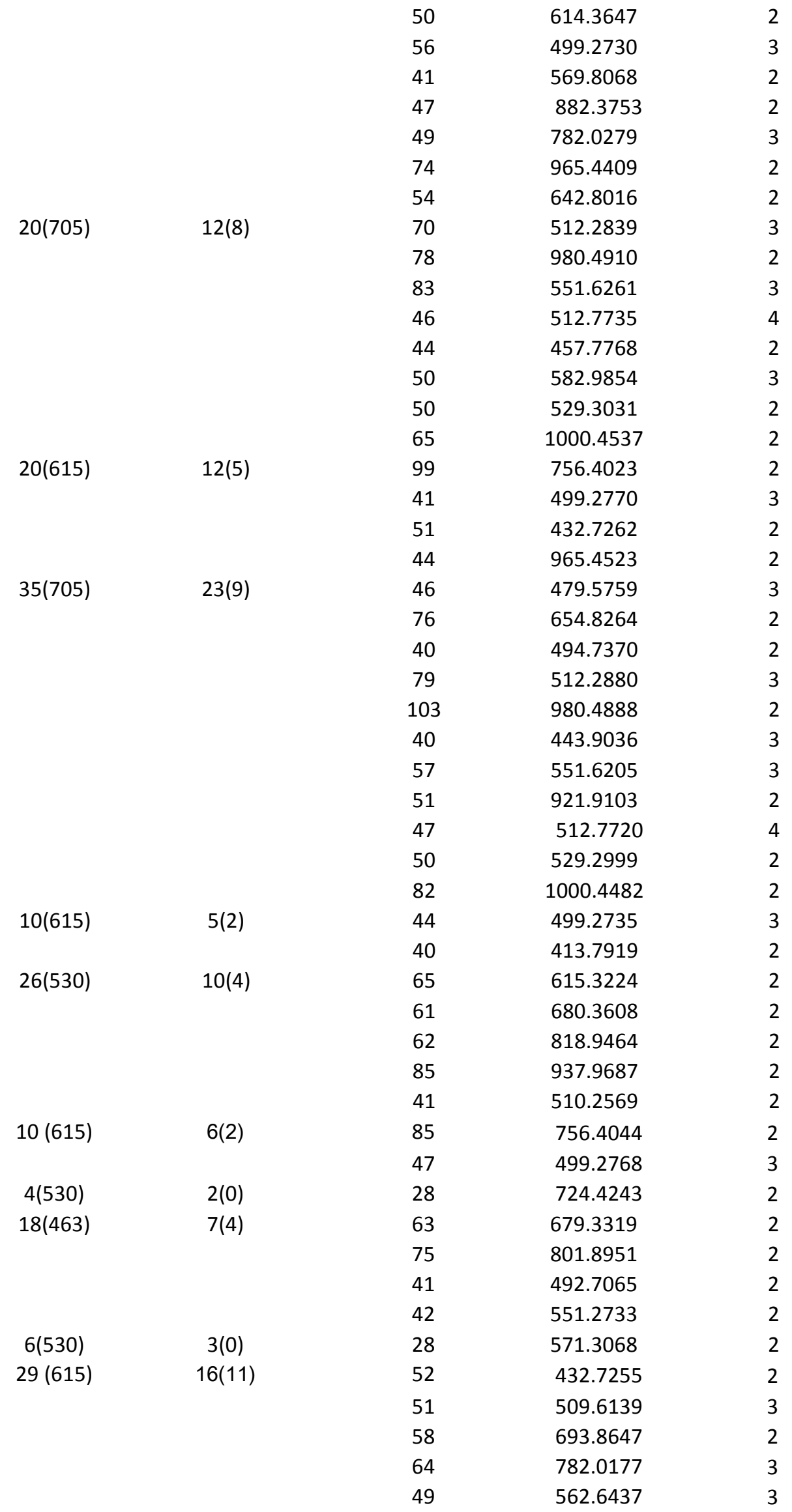




\begin{tabular}{|c|c|c|c|c|}
\hline & & 73 & 779.9160 & 2 \\
\hline & & 68 & 671.3034 & 3 \\
\hline & & 69 & 965.4438 & 2 \\
\hline & & 43 & 413.7920 & 2 \\
\hline & & 75 & 634.8142 & 2 \\
\hline & & 73 & 746.6940 & 3 \\
\hline $14(434)$ & $3(1)$ & 46 & 756.4090 & 4 \\
\hline 30 (615) & $18(9)$ & 47 & 432.7271 & 2 \\
\hline & & 60 & 516.2631 & 3 \\
\hline & & 48 & 509.6154 & 3 \\
\hline & & 53 & 562.6497 & 3 \\
\hline & & 73 & 779.4127 & 2 \\
\hline & & 47 & 671.3115 & 3 \\
\hline & & 49 & 466.8885 & 3 \\
\hline & & 57 & 965.4287 & 2 \\
\hline & & 43 & 413.7927 & 2 \\
\hline 26 (615) & $17(3)$ & 55 & 516.2650 & 3 \\
\hline & & 40 & 782.3465 & 3 \\
\hline & & 47 & 965.4367 & 2 \\
\hline $14(381)$ & $4(3)$ & 51 & 616.8226 & 2 \\
\hline & & 71 & 679.8839 & 2 \\
\hline & & 63 & 779.4074 & 2 \\
\hline $27(381)$ & $8(4)$ & 62 & 616.8123 & 2 \\
\hline & & 71 & 679.8792 & 2 \\
\hline & & 44 & 424.7517 & 2 \\
\hline & & 76 & 707.6952 & 3 \\
\hline 19(416) & $8(6)$ & 55 & 590.8030 & 2 \\
\hline & & 73 & 668.3715 & 2 \\
\hline & & 46 & 779.3771 & 2 \\
\hline & & 64 & 493.7200 & 2 \\
\hline & & 53 & 460.7585 & 2 \\
\hline & & 89 & 779.4235 & 2 \\
\hline $45(68)$ & $2(1)$ & 94 & 859.7257 & 3 \\
\hline $3(298)$ & $1(0)$ & 36 & 428.7679 & 2 \\
\hline 66 (434) & $24(16)$ & 86 & 725.3659 & 2 \\
\hline & & 148 & 902.9781 & 2 \\
\hline & & 65 & 707.8737 & 2 \\
\hline & & 58 & 556.9332 & 3 \\
\hline & & 68 & 648.7914 & 2 \\
\hline & & 71 & 760.4161 & 2 \\
\hline & & 120 & 1007.8672 & 3 \\
\hline & & 51 & 572.3112 & 2 \\
\hline & & 88 & 980.9700 & 2 \\
\hline & & 50 & 408.2495 & 2 \\
\hline & & 108 & 786.8888 & 2 \\
\hline & & 104 & 841.7252 & 3 \\
\hline & & 82 & 1031.0492 & 2 \\
\hline & & 40 & 504.2546 & 4 \\
\hline
\end{tabular}




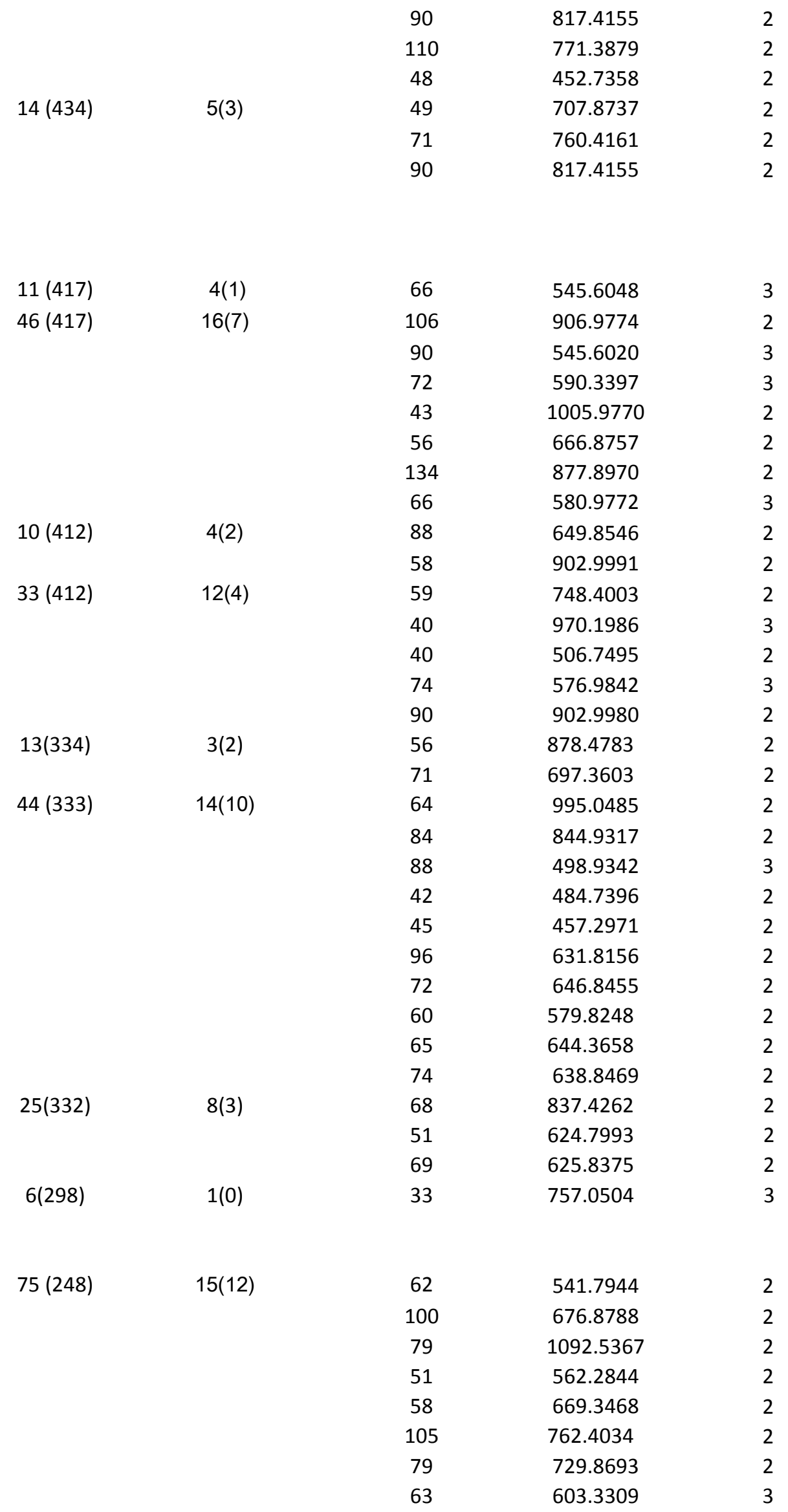




\begin{tabular}{|c|c|c|c|c|}
\hline & & 83 & 801.9528 & 2 \\
\hline & & 54 & 489.5830 & 3 \\
\hline & & 74 & 629.3148 & 2 \\
\hline & & 54 & 1023.8688 & 3 \\
\hline $25(220)$ & $4(2)$ & 54 & 945.0143 & 2 \\
\hline & & 62 & 508.3004 & 2 \\
\hline $17(264)$ & $4(2)$ & 94 & 687.3686 & 2 \\
\hline & & 43 & 493.2797 & 2 \\
\hline $56(248)$ & $12(8)$ & 44 & 728.6894 & 3 \\
\hline & & 59 & 669.3375 & 2 \\
\hline & & 51 & 508.6071 & 3 \\
\hline & & 61 & 486.9175 & 3 \\
\hline & & 77 & 801.9498 & 2 \\
\hline & & 65 & 733.8696 & 2 \\
\hline & & 83 & 629.3167 & 2 \\
\hline & & 57 & 663.3313 & 2 \\
\hline 70 (150) & $8(7)$ & 43 & 472.6159 & 3 \\
\hline & & 52 & 499.2446 & 3 \\
\hline & & 89 & 684.3175 & 2 \\
\hline & & 104 & 872.0824 & 3 \\
\hline & & 70 & 631.2918 & 2 \\
\hline & & 92 & 830.3990 & 3 \\
\hline & & 54 & 866.4220 & 3 \\
\hline $25(264)$ & $6(2)$ & 74 & 661.8323 & 2 \\
\hline & & 88 & 687.3679 & 2 \\
\hline & & 41 & 603.3400 & 2 \\
\hline $4(615)$ & $3(1)$ & 54 & 499.2742 & 3 \\
\hline $6(150)$ & $1(1)$ & 49 & 631.2902 & 2 \\
\hline $48(321)$ & $16(6)$ & 67 & 634.9478 & 2 \\
\hline & & 64 & 501.4083 & 2 \\
\hline & & 40 & 500.3796 & 2 \\
\hline & & 44 & 616.9331 & 2 \\
\hline & & 63 & 885.6116 & 2 \\
\hline & & 52 & 537.4057 & 2 \\
\hline $55(264)$ & $17(10)$ & 54 & 664.3240 & 2 \\
\hline & & 79 & 661.8341 & 2 \\
\hline & & 92 & 687.3576 & 2 \\
\hline & & 63 & 441.2466 & 3 \\
\hline & & 44 & 500.2981 & 2 \\
\hline & & 57 & 493.2700 & 2 \\
\hline & & 48 & 589.2941 & 2 \\
\hline & & 52 & 596.3070 & 2 \\
\hline & & 52 & 568.7845 & 2 \\
\hline & & 55 & 603.3375 & 2 \\
\hline $19(103)$ & $2(1)$ & 76 & 635.8704 & 2 \\
\hline $61(264)$ & $16(12)$ & 62 & 878.4097 & 2 \\
\hline & & 66 & 663.8261 & 2 \\
\hline & & 77 & 661.8364 & 2 \\
\hline
\end{tabular}




\begin{tabular}{|c|c|c|c|c|}
\hline & & 98 & 687.3710 & 2 \\
\hline & & 63 & 441.2487 & 3 \\
\hline & & 49 & 627.2874 & 2 \\
\hline & & 64 & 493.2666 & 2 \\
\hline & & 53 & 589.2977 & 2 \\
\hline & & 49 & 596.3005 & 2 \\
\hline & & 53 & 553.2861 & 2 \\
\hline & & 54 & 568.7929 & 2 \\
\hline & & 45 & 603.3370 & 2 \\
\hline $11(103)$ & $1(1)$ & 76 & 635.8797 & 2 \\
\hline $26(615)$ & $14(8)$ & 68 & 643.8200 & 2 \\
\hline & & 89 & 756.4039 & 2 \\
\hline & & 67 & 614.3603 & 2 \\
\hline & & 89 & 1038.8342 & 3 \\
\hline & & 40 & 690.7595 & 2 \\
\hline & & 77 & 748.4039 & 2 \\
\hline & & 105 & 1026.5177 & 3 \\
\hline & & 88 & 974.5045 & 3 \\
\hline & & 76 & 597.7755 & 2 \\
\hline 36 (103) & $2(1)$ & 76 & 635.8697 & 2 \\
\hline 44 (103) & $3(3)$ & 76 & 636.0034 & 2 \\
\hline & & 54 & 729.2132 & 3 \\
\hline & & 76 & 980.3604 & 3 \\
\hline 31 (264) & $8(4)$ & 53 & 661.9675 & 2 \\
\hline & & 44 & 687.5035 & 2 \\
\hline & & 47 & 568.9066 & 2 \\
\hline & & 41 & 603.4650 & 2 \\
\hline $4(183)$ & $1(1)$ & 50 & 487.8503 & 2 \\
\hline $17(615)$ & $9(4)$ & 65 & 643.8131 & 2 \\
\hline & & 93 & 764.4051 & 2 \\
\hline & & 44 & 472.2124 & 2 \\
\hline & & 46 & 499.2736 & 3 \\
\hline $10(615)$ & $6(2)$ & 76 & 764.4047 & 2 \\
\hline & & 59 & 597.7727 & 2 \\
\hline 47 (196) & $9(4)$ & 52 & 599.8185 & , \\
\hline & & 108 & 1048.9492 & 2 \\
\hline & & 41 & 516.7593 & 2 \\
\hline & & 59 & 580.8111 & 2 \\
\hline $3(264)$ & $1(1)$ & 44 & 603.3416 & 2 \\
\hline $54(150)$ & $7(5)$ & 65 & 472.6164 & 3 \\
\hline & & 84 & 684.3135 & 2 \\
\hline & & 96 & 872.0815 & 3 \\
\hline & & 70 & 631.2880 & 2 \\
\hline & & 74 & 830.3983 & 3 \\
\hline 69 (132) & 9(9) & 64 & 743.3176 & 2 \\
\hline & & 52 & 469.2515 & 2 \\
\hline & & 70 & 624.6753 & 3 \\
\hline & & 65 & 780.4484 & 3 \\
\hline
\end{tabular}




\begin{tabular}{|c|c|c|c|}
\hline $8(150)$ & 1(1) & 50 & 684.3157 \\
\hline \multirow[t]{5}{*}{$53(132)$} & $5(4)$ & 44 & 490.5538 \\
\hline & & 55 & 469.2500 \\
\hline & & 51 & 585.5839 \\
\hline & & 110 & 762.3264 \\
\hline & & 96 & 785.8959 \\
\hline \multirow[t]{5}{*}{58 (139) } & $7(5)$ & 102 & 911.4720 \\
\hline & & 58 & 665.3203 \\
\hline & & 45 & 450.7876 \\
\hline & & 53 & 545.8190 \\
\hline & & 52 & 694.9491 \\
\hline \multirow[t]{4}{*}{$92(55)$} & $5(4)$ & 43 & 444.2158 \\
\hline & & 58 & 599.7471 \\
\hline & & 67 & 1028.9530 \\
\hline & & 90 & 934.7728 \\
\hline \multirow[t]{4}{*}{$76(55)$} & $4(4)$ & 44 & 444.2272 \\
\hline & & 48 & 599.7596 \\
\hline & & 55 & 1028.9514 \\
\hline & & 133 & 934.7765 \\
\hline 19 (705) & $8(1)$ & 63 & 942.8284 \\
\hline $32(67)$ & 2(1) & 61 & 538.2657 \\
\hline $45(68)$ & $2(1)$ & 73 & 859.7254 \\
\hline \multirow[t]{2}{*}{$45(68)$} & $2(2)$ & 41 & 441.6988 \\
\hline & & 109 & 859.7216 \\
\hline $45(68)$ & 2(1) & 97 & 859.7217 \\
\hline $32(67)$ & $2(1)$ & 49 & 538.2725 \\
\hline $45(68)$ & $2(1)$ & 94 & 860.0636 \\
\hline $615(11)$ & $7(1)$ & 60 & 752.67 \\
\hline 615(9) & $4(1)$ & 63 & 752.66 \\
\hline \multirow[t]{12}{*}{$615(58)$} & $34(27)$ & 68 & 644.33 \\
\hline & & 55 & 695.62 \\
\hline & & 91 & 756.42 \\
\hline & & 40 & 472.22 \\
\hline & & 89 & 1038.84 \\
\hline & & 44 & 806.88 \\
\hline & & 86 & 748.91 \\
\hline & & 47 & 770.14 \\
\hline & & 51 & 731.11 \\
\hline & & 108 & 846.08 \\
\hline & & 109 & 1128.49 \\
\hline & & 67 & 509.61 \\
\hline
\end{tabular}




\begin{tabular}{|c|c|c|c|c|}
\hline & & 67 & 685.86 & 2 \\
\hline & & 75 & 782.03 & 3 \\
\hline & & 85 & 835.46 & 2 \\
\hline & & 73 & 771.42 & 2 \\
\hline & & 56 & 557.32 & 3 \\
\hline & & 78 & 671.31 & 3 \\
\hline & & 77 & 965.44 & 2 \\
\hline & & 44 & 516.64 & 2 \\
\hline & & 44 & 463.59 & 3 \\
\hline & & 94 & 634.96 & 2 \\
\hline & & 122 & 746.69 & 3 \\
\hline $615(56)$ & $31(21)$ & 45 & 643.82 & 2 \\
\hline & & 61 & 695.61 & 4 \\
\hline & & 95 & 756.40 & 2 \\
\hline & & 101 & 1038.83 & 3 \\
\hline & & 84 & 748.40 & 2 \\
\hline & & 77 & 597.77 & 2 \\
\hline & & 43 & 432.73 & 2 \\
\hline & & 42 & 569.80 & 2 \\
\hline & & 68 & 752.66 & 3 \\
\hline & & 53 & 509.61 & 3 \\
\hline & & 81 & 782.03 & 3 \\
\hline & & 58 & 835.46 & 2 \\
\hline & & 76 & 771.42 & 2 \\
\hline & & 56 & 557.31 & 3 \\
\hline & & 65 & 671.31 & 3 \\
\hline & & 89 & 965.44 & 2 \\
\hline & & 54 & 516.75 & 2 \\
\hline & & 43 & 413.79 & 2 \\
\hline & & 58 & 616.32 & 2 \\
\hline & & 80 & 634.82 & 2 \\
\hline & & 122 & 746.69 & 3 \\
\hline $705(3)$ & $2(0)$ & 34 & 565.94 & 3 \\
\hline $446(2)$ & $1(0)$ & 22 & 733.41 & 2 \\
\hline $615(45)$ & $30(21)$ & 74 & 695.61 & 4 \\
\hline & & 92 & 718.05 & 3 \\
\hline & & 56 & 409.92 & 3 \\
\hline & & 74 & 1038.83 & 3 \\
\hline & & 100 & 846.67 & 4 \\
\hline & & 64 & 748.40 & 2 \\
\hline & & 74 & 770.14 & 4 \\
\hline & & 65 & 591.05 & 4 \\
\hline & & 59 & 690.90 & 2 \\
\hline & & 48 & 432.73 & 2 \\
\hline & & 49 & 516.27 & 3 \\
\hline & & 58 & 782.03 & 3 \\
\hline & & 41 & 562.64 & 3 \\
\hline & & 47 & 557.31 & 3 \\
\hline & & 76 & 491.84 & 2 \\
\hline & & 157 & 1119.53 & 2 \\
\hline
\end{tabular}




\begin{tabular}{|c|c|c|c|c|}
\hline \multirow[t]{9}{*}{$615(31)$} & \multirow[t]{9}{*}{$17(9)$} & 56 & 643.82 & 2 \\
\hline & & 79 & 756.40 & 2 \\
\hline & & 69 & 499.27 & 3 \\
\hline & & 78 & 782.02 & 3 \\
\hline & & 88 & 779.42 & 2 \\
\hline & & 62 & 965.44 & 2 \\
\hline & & 57 & 516.75 & 2 \\
\hline & & 41 & 413.79 & 2 \\
\hline & & 74 & 634.82 & 2 \\
\hline \multirow[t]{7}{*}{$705(14)$} & \multirow[t]{7}{*}{$9(6)$} & 65 & 512.29 & 3 \\
\hline & & 56 & 980.49 & 2 \\
\hline & & 46 & 612.32 & 2 \\
\hline & & 52 & 534.26 & 2 \\
\hline & & 49 & 401.21 & 3 \\
\hline & & 42 & 457.25 & 2 \\
\hline & & 39 & 551.62 & 3 \\
\hline $446(2)$ & $1(1)$ & 53 & 733.42 & 2 \\
\hline \multirow[t]{13}{*}{ 705(33) } & \multirow[t]{13}{*}{$21(13)$} & 55 & 479.59 & 3 \\
\hline & & 54 & 512.29 & 3 \\
\hline & & 78 & 980.49 & 2 \\
\hline & & 88 & 821.40 & 2 \\
\hline & & 56 & 557.93 & 3 \\
\hline & & 60 & 612.32 & 2 \\
\hline & & 52 & 534.26 & 2 \\
\hline & & 52 & 401.21 & 3 \\
\hline & & 61 & 551.62 & 3 \\
\hline & & 80 & 822.40 & 2 \\
\hline & & 73 & 921.91 & 2 \\
\hline & & 65 & 512.77 & 4 \\
\hline & & 50 & 529.30 & 2 \\
\hline \multirow[t]{3}{*}{ 446(10) } & \multirow[t]{3}{*}{$3(3)$} & 46 & 498.73 & 2 \\
\hline & & 79 & 733.42 & 2 \\
\hline & & 49 & 880.45 & 3 \\
\hline $615(7)$ & $5(1)$ & 47 & 413.79 & 2 \\
\hline \multirow[t]{13}{*}{$705(28)$} & \multirow[t]{13}{*}{ 20(13) } & 50 & 479.59 & 3 \\
\hline & & 76 & 654.83 & 2 \\
\hline & & 70 & 512.29 & 3 \\
\hline & & 88 & 980.49 & 2 \\
\hline & & 54 & 821.40 & 2 \\
\hline & & 42 & 565.95 & 3 \\
\hline & & 62 & 612.32 & 2 \\
\hline & & 52 & 534.26 & 2 \\
\hline & & 62 & 401.21 & 3 \\
\hline & & 47 & 551.62 & 3 \\
\hline & & 83 & 822.40 & 2 \\
\hline & & 56 & 512.77 & 4 \\
\hline & & 50 & 529.30 & 2 \\
\hline $446(2)$ & $1(1)$ & 81 & 733.44 & 2 \\
\hline $615(1)$ & 1(1) & 56 & 413.79 & 2 \\
\hline
\end{tabular}




\begin{tabular}{|c|c|c|c|c|}
\hline \multirow[t]{10}{*}{$705(21)$} & \multirow[t]{10}{*}{$13(9)$} & 54 & 558.95 & 3 \\
\hline & & 72 & 654.82 & 2 \\
\hline & & 80 & 512.29 & 3 \\
\hline & & 48 & 980.49 & 2 \\
\hline & & 73 & 557.93 & 3 \\
\hline & & 48 & 534.26 & 2 \\
\hline & & 55 & 443.90 & 3 \\
\hline & & 43 & 457.24 & 2 \\
\hline & & 92 & 826.93 & 2 \\
\hline & & 41 & 457.78 & 2 \\
\hline \multirow[t]{8}{*}{ 778(13) } & \multirow[t]{8}{*}{$9(8)$} & 44 & 418.91 & 3 \\
\hline & & 59 & 499.78 & 2 \\
\hline & & 60 & 642.88 & 2 \\
\hline & & 67 & 639.36 & 2 \\
\hline & & 52 & 637.34 & 3 \\
\hline & & 46 & 562.31 & 3 \\
\hline & & 81 & 610.83 & 2 \\
\hline & & 80 & 752.39 & 2 \\
\hline \multirow[t]{5}{*}{ 705(10) } & \multirow[t]{5}{*}{$7(4)$} & 69 & 512.29 & 3 \\
\hline & & 66 & 612.32 & 2 \\
\hline & & 50 & 401.21 & 3 \\
\hline & & 41 & 457.24 & 2 \\
\hline & & 43 & 512.77 & 4 \\
\hline \multirow[t]{2}{*}{$705(6)$} & \multirow[t]{2}{*}{$4(2)$} & 61 & 821.40 & 2 \\
\hline & & 41 & 551.62 & 3 \\
\hline $250(14)$ & $4(1)$ & 61 & 649.87 & 2 \\
\hline \multirow[t]{16}{*}{ 705(36) } & \multirow[t]{16}{*}{$26(20)$} & 110 & 718.88 & 2 \\
\hline & & 81 & 654.83 & 2 \\
\hline & & 74 & 767.93 & 2 \\
\hline & & 92 & 980.49 & 2 \\
\hline & & 72 & 821.40 & 2 \\
\hline & & 87 & 557.93 & 3 \\
\hline & & 61 & 518.28 & 2 \\
\hline & & 59 & 612.32 & 2 \\
\hline & & 62 & 401.21 & 3 \\
\hline & & 62 & 443.91 & 3 \\
\hline & & 72 & 826.92 & 2 \\
\hline & & 50 & 921.91 & 2 \\
\hline & & 42 & 442.70 & 2 \\
\hline & & 57 & 512.77 & 4 \\
\hline & & 50 & 529.30 & 2 \\
\hline & & 54 & 672.64 & 3 \\
\hline \multirow[t]{7}{*}{ 705(32) } & \multirow[t]{7}{*}{$22(7)$} & 51 & 602.30 & 4 \\
\hline & & 70 & 654.83 & 2 \\
\hline & & 72 & 512.29 & 3 \\
\hline & & 96 & 980.49 & 2 \\
\hline & & 64 & 691.01 & 3 \\
\hline & & 82 & 826.93 & 2 \\
\hline & & 80 & 822.40 & 2 \\
\hline $705(22)$ & $15(7)$ & 82 & 767.93 & 2 \\
\hline
\end{tabular}




\begin{tabular}{|c|c|c|c|c|}
\hline & & 83 & 980.48 & 2 \\
\hline & & 80 & 848.42 & 2 \\
\hline & & 62 & 612.32 & 2 \\
\hline & & 71 & 551.62 & 3 \\
\hline & & 50 & 512.77 & 4 \\
\hline & & 49 & 582.99 & 3 \\
\hline $615(11)$ & $7(0)$ & 40 & 471.97 & 4 \\
\hline $615(3)$ & $2(2)$ & 50 & 432.73 & 2 \\
\hline & & 57 & 625.86 & 2 \\
\hline 615(37) & $20(8)$ & 84 & 756.42 & 2 \\
\hline & & 53 & 499.27 & 3 \\
\hline & & 49 & 432.73 & 2 \\
\hline & & 67 & 851.09 & 3 \\
\hline & & 59 & 782.03 & 3 \\
\hline & & 76 & 771.42 & 2 \\
\hline & & 54 & 965.44 & 2 \\
\hline & & 61 & 634.82 & 2 \\
\hline $615(27)$ & $17(8)$ & 85 & 756.41 & 2 \\
\hline & & 46 & 499.28 & 3 \\
\hline & & 72 & 597.77 & 2 \\
\hline & & 41 & 460.93 & 3 \\
\hline & & 48 & 432.73 & 2 \\
\hline & & 50 & 752.66 & 3 \\
\hline & & 42 & 454.26 & 4 \\
\hline & & 55 & 516.74 & 2 \\
\hline $778(2)$ & $2(1)$ & 67 & 610.82 & 2 \\
\hline $615(4)$ & $3(0)$ & 40 & 432.72 & 2 \\
\hline $472(20)$ & $6(2)$ & 104 & 1028.79 & 3 \\
\hline & & 45 & 721.74 & 5 \\
\hline & $10(6)$ & 56 & 616.81 & 2 \\
\hline & & 81 & 679.88 & 2 \\
\hline & & 79 & 779.40 & 2 \\
\hline & & 49 & 424.75 & 2 \\
\hline & & 44 & 446.25 & 2 \\
\hline & & 59 & 659.82 & 2 \\
\hline $416(2)$ & $1(0)$ & 39 & 754.39 & 2 \\
\hline $434(55)$ & $19(11)$ & 69 & 641.67 & 3 \\
\hline & & 114 & 902.97 & 2 \\
\hline & & 78 & 634.33 & 4 \\
\hline & & 77 & 756.15 & 4 \\
\hline & & 50 & 572.31 & 2 \\
\hline & & 88 & 919.80 & 3 \\
\hline & & 46 & 408.25 & 2 \\
\hline & & 76 & 786.89 & 2 \\
\hline & & 52 & 618.95 & 3 \\
\hline & & 68 & 519.93 & 3 \\
\hline & & 44 & 452.74 & 2 \\
\hline 434(15) & $4(0)$ & 30 & 817.42 & 2 \\
\hline
\end{tabular}




\begin{tabular}{|c|c|c|c|c|}
\hline \multirow[t]{5}{*}{$150(28)$} & \multirow[t]{5}{*}{$7(5)$} & 51 & 783.95 & 2 \\
\hline & & 50 & 429.92 & 3 \\
\hline & & 65 & 472.62 & 3 \\
\hline & & 87 & 684.32 & 2 \\
\hline & & 75 & 631.29 & 2 \\
\hline $334(17)$ & $6(1)$ & 54 & 697.36 & 2 \\
\hline $333(13)$ & $3(0)$ & 27 & 658.36 & 3 \\
\hline \multirow[t]{12}{*}{$333(57)$} & $13(12)$ & 83 & 727.92 & 2 \\
\hline & & 67 & 663.70 & 3 \\
\hline & & 86 & 844.93 & 2 \\
\hline & & 43 & 493.74 & 4 \\
\hline & & 91 & 498.93 & 3 \\
\hline & & 46 & 457.30 & 2 \\
\hline & & 44 & 894.80 & 3 \\
\hline & & 82 & 631.81 & 2 \\
\hline & & 72 & 646.85 & 2 \\
\hline & & 42 & 485.29 & 2 \\
\hline & & 54 & 1028.20 & 3 \\
\hline & & 63 & 574.80 & 2 \\
\hline \multirow[t]{5}{*}{$409(26)$} & $9(5)$ & 42 & 622.75 & 2 \\
\hline & & 48 & 538.79 & 2 \\
\hline & & 52 & 549.31 & 3 \\
\hline & & 59 & 515.30 & 2 \\
\hline & & 63 & 691.85 & 2 \\
\hline $333(6)$ & $2(1)$ & 41 & 403.22 & 2 \\
\hline \multirow[t]{12}{*}{$264(61)$} & $18(13)$ & 50 & 656.33 & 2 \\
\hline & & 82 & 661.84 & 2 \\
\hline & & 102 & 687.37 & 2 \\
\hline & & 64 & 441.25 & 3 \\
\hline & & 50 & 627.30 & 2 \\
\hline & & 46 & 500.31 & 2 \\
\hline & & 57 & 493.28 & 2 \\
\hline & & 57 & 589.30 & 2 \\
\hline & & 49 & 596.31 & 2 \\
\hline & & 57 & 545.29 & 2 \\
\hline & & 61 & 560.80 & 2 \\
\hline & & 55 & 603.35 & 2 \\
\hline \multirow[t]{2}{*}{$103(28)$} & $3(2)$ & 77 & 635.87 & 2 \\
\hline & & 65 & 729.06 & 3 \\
\hline \multirow[t]{2}{*}{$264(25)$} & $7(2)$ & 62 & 661.84 & 2 \\
\hline & & 78 & 687.37 & 2 \\
\hline \multirow[t]{2}{*}{$248(33)$} & $7(2)$ & 61 & 661.35 & 2 \\
\hline & & 54 & 486.91 & 3 \\
\hline 196(22) & $4(3)$ & 56 & 599.81 & 2 \\
\hline
\end{tabular}




\begin{tabular}{|c|c|c|c|c|}
\hline & & 71 & 1048.95 & 2 \\
\hline & & 44 & 516.76 & 2 \\
\hline \multirow[t]{2}{*}{$264(23)$} & $6(2)$ & 64 & 687.37 & 2 \\
\hline & & 50 & 560.79 & 2 \\
\hline \multirow[t]{3}{*}{$150(52)$} & $5(3)$ & 97 & 684.32 & 2 \\
\hline & & 91 & 872.09 & 3 \\
\hline & & 75 & 631.29 & 2 \\
\hline \multirow[t]{9}{*}{$132(80)$} & $12(8)$ & 56 & 490.55 & 3 \\
\hline & & 58 & 461.25 & 2 \\
\hline & & 58 & 469.24 & 2 \\
\hline & & 49 & 585.59 & 4 \\
\hline & & 97 & 510.93 & 3 \\
\hline & & 48 & 419.72 & 2 \\
\hline & & 110 & 762.33 & 2 \\
\hline & & 55 & 546.65 & 3 \\
\hline & & 83 & 785.90 & 2 \\
\hline \multirow[t]{3}{*}{$139(46)$} & $5(3)$ & 57 & 665.32 & 2 \\
\hline & & 43 & 450.79 & 2 \\
\hline & & 52 & 545.82 & 2 \\
\hline \multirow[t]{3}{*}{$55(76)$} & $3(2)$ & 42 & 444.22 & 2 \\
\hline & & 55 & 599.76 & 2 \\
\hline & & 73 & 934.77 & 3 \\
\hline \multirow[t]{2}{*}{$55(76)$} & $3(2)$ & 62 & 599.76 & 2 \\
\hline & & 118 & 934.77 & 3 \\
\hline $67(14)$ & $1(1)$ & 62 & 538.26 & 2 \\
\hline $2(1193)$ & $3(1)$ & 80 & 842.8748 & 2 \\
\hline $2(816)$ & $2(1)$ & 55 & 684.3533 & 2 \\
\hline \multirow[t]{4}{*}{$13(816)$} & $9(6)$ & 44 & 527.3690 & 2 \\
\hline & & 68 & 684.4747 & 2 \\
\hline & & 67 & 692.9279 & 2 \\
\hline & & 71 & 741.0018 & 2 \\
\hline $3(272)$ & $1(0)$ & 27 & 499.2887 & 2 \\
\hline \multirow[t]{5}{*}{$21(615)$} & $11(5)$ & 46 & 1038.8198 & 3 \\
\hline & & 59 & 499.2779 & 3 \\
\hline & & 42 & 569.8094 & 2 \\
\hline & & 69 & 516.2676 & 3 \\
\hline & & 43 & 514.9502 & 3 \\
\hline $3(471)$ & 1(1) & 77 & 828.9789 & 2 \\
\hline
\end{tabular}




\begin{tabular}{|c|c|c|c|c|}
\hline \multirow[t]{3}{*}{$23(615)$} & \multirow[t]{3}{*}{$11(3)$} & 57 & 1038.8359 & 3 \\
\hline & & 77 & 597.7697 & 2 \\
\hline & & 45 & 413.7937 & 2 \\
\hline 1 (816) & 1(1) & 42 & 527.2764 & 2 \\
\hline $10(105)$ & $1(0)$ & 35 & 680.8566 & 2 \\
\hline \multirow[t]{25}{*}{65 (615) } & \multirow[t]{25}{*}{$39(28)$} & 68 & 643.8323 & 2 \\
\hline & & 41 & 695.6096 & 4 \\
\hline & & 88 & 756.4096 & 2 \\
\hline & & 87 & 614.3708 & 2 \\
\hline & & 129 & 1038.8364 & 3 \\
\hline & & 88 & 748.4461 & 2 \\
\hline & & 93 & 974.4708 & 3 \\
\hline & & 69 & 591.0511 & 4 \\
\hline & & 43 & 432.7206 & 2 \\
\hline & & 46 & 569.8126 & 2 \\
\hline & & 98 & 874.3769 & 2 \\
\hline & & 60 & 516.2698 & 3 \\
\hline & & 87 & 846.0706 & 3 \\
\hline & & 111 & 752.3622 & 3 \\
\hline & & 79 & 763.9162 & 2 \\
\hline & & 69 & 685.8668 & 2 \\
\hline & & 60 & 835.4627 & 2 \\
\hline & & 78 & 600.0102 & 2 \\
\hline & & 81 & 771.4370 & 2 \\
\hline & & 55 & 557.3165 & 3 \\
\hline & & 66 & 671.3064 & 3 \\
\hline & & 78 & 692.3413 & 2 \\
\hline & & 68 & 973.5501 & 2 \\
\hline & & 90 & 634.8193 & 2 \\
\hline & & 146 & 1119.5289 & 2 \\
\hline \multirow[t]{17}{*}{$56(445)$} & \multirow[t]{17}{*}{$21(17)$} & 48 & 709.3642 & 3 \\
\hline & & 47 & 608.3164 & 3 \\
\hline & & 90 & 933.5420 & 3 \\
\hline & & 139 & 980.0155 & 2 \\
\hline & & 91 & 696.3518 & 3 \\
\hline & & 77 & 903.4600 & 3 \\
\hline & & 75 & 565.8029 & 2 \\
\hline & & 57 & 629.8457 & 2 \\
\hline & & 45 & 429.9126 & 3 \\
\hline & & 68 & 580.3229 & 2 \\
\hline & & 62 & 546.6139 & 3 \\
\hline & & 47 & 731.8485 & 2 \\
\hline & & 46 & 848.9089 & 2 \\
\hline & & 61 & 514.7651 & 2 \\
\hline & & 111 & 937.5253 & 2 \\
\hline & & 41 & 677.3504 & 3 \\
\hline & & 52 & 623.3001 & 2 \\
\hline \multirow[t]{3}{*}{$26(446)$} & \multirow[t]{3}{*}{$13(10)$} & 67 & 809.4177 & 2 \\
\hline & & 139 & 980.0155 & 2 \\
\hline & & 91 & 696.3518 & 3 \\
\hline
\end{tabular}




\begin{tabular}{|c|c|c|c|}
\hline & & 75 & 565.8029 \\
\hline & & 57 & 629.8457 \\
\hline & & 45 & 429.9126 \\
\hline & & 68 & 580.3229 \\
\hline & & 47 & 854.4335 \\
\hline & & 46 & 848.9089 \\
\hline & & 102 & 937.5253 \\
\hline & & 40 & 677.3504 \\
\hline 16 (448) & $6(3)$ & 53 & 705.8893 \\
\hline & & 63 & 573.6351 \\
\hline & & 80 & 1205.1168 \\
\hline 20 (448) & $6(5)$ & 77 & 705.8897 \\
\hline & & 73 & 573.6366 \\
\hline & & 119 & 1205.1106 \\
\hline & & 44 & 508.2969 \\
\hline & & 51 & 777.3502 \\
\hline $14(446)$ & $5(3)$ & 89 & 653.6599 \\
\hline & & 71 & 565.8043 \\
\hline & & 74 & 580.3255 \\
\hline
\end{tabular}

$\begin{array}{ccccc}19(778) & 15(8) & 57 & 411.2188 & 2 \\ & & 63 & 639.3536 & 2 \\ & 57 & 561.7749 & 2 \\ & 65 & 637.3400 & 3 \\ & 43 & 438.9490 & 3 \\ & & 1051.5497 & 2 \\ 17(705) & 60 & 666.8328 & 2 \\ & & 47 & 526.2588 & 2 \\ 6(615) & 10(3) & 41 & 821.4005 & 2 \\ 35(778) & & 534.3088 & 2 \\ & & 43 & 457.3075 & 2 \\ & 4(0) & 32 & 413.7892 & 2 \\ & & 46 & 418.9009 & 3 \\ & & 59 & 499.7715 & 2 \\ & & 75 & 642.8794 & 2 \\ & & 113 & 1104.5557 & 2 \\ & & 922.4487 & 2 \\ & & 49 & 411.2166 & 2 \\ & & 65 & 673.3352 & 3 \\ & & 52 & 562.3317 & 3 \\ & & 44 & 338.9000 & 3 \\ & & 81 & 610.8237 & 2 \\ & & 75 & 752.3815 & 2\end{array}$




\begin{tabular}{|c|c|c|c|c|}
\hline & & 57 & 666.8347 & 2 \\
\hline & & 47 & 464.2326 & 3 \\
\hline & & 55 & 526.2634 & 2 \\
\hline \multirow[t]{3}{*}{$9(615)$} & $6(3)$ & 73 & 643.8195 & 2 \\
\hline & & 41 & 756.4102 & 2 \\
\hline & & 49 & 413.7904 & 2 \\
\hline $2(446)$ & 1(1) & 65 & 733.4259 & 2 \\
\hline \multirow[t]{2}{*}{$13(250)$} & $2(2)$ & 44 & 504.7746 & 2 \\
\hline & & 70 & 871.7680 & 3 \\
\hline \multirow[t]{2}{*}{$3(705)$} & $3(2)$ & 51 & 601.3734 & 2 \\
\hline & & 44 & 457.2372 & 2 \\
\hline \multirow[t]{25}{*}{$54(705)$} & $34(31)$ & 129 & 718.8753 & 2 \\
\hline & & 81 & 654.8723 & 2 \\
\hline & & 92 & 768.0234 & 2 \\
\hline & & 87 & 980.4935 & 2 \\
\hline & & 90 & 821.4114 & 2 \\
\hline & & 57 & 557.9359 & 3 \\
\hline & & 58 & 518.4788 & 2 \\
\hline & & 43 & 677.3546 & 2 \\
\hline & & 116 & 848.5256 & 2 \\
\hline & & 61 & 612.3897 & 2 \\
\hline & & 51 & 534.8087 & 2 \\
\hline & & 57 & 401.2062 & 3 \\
\hline & & 58 & 443.9172 & 3 \\
\hline & & 54 & 471.3103 & 4 \\
\hline & & 51 & 594.4265 & 2 \\
\hline & & 46 & 649.4585 & 3 \\
\hline & & 40 & 905.4308 & 3 \\
\hline & & 63 & 521.8361 & 2 \\
\hline & & 72 & 551.7640 & 3 \\
\hline & & 63 & 630.3085 & 3 \\
\hline & & 84 & 921.9050 & 2 \\
\hline & & 48 & 546.8216 & 2 \\
\hline & & 108 & 874.0862 & 2 \\
\hline & & 50 & 529.8214 & 2 \\
\hline & & 90 & 1008.4531 & 2 \\
\hline \multirow[t]{2}{*}{$4(615)$} & $3(2)$ & 96 & 756.4614 & 2 \\
\hline & & 46 & 413.8023 & 2 \\
\hline $2(446)$ & $1(1)$ & 80 & 733.4910 & 2 \\
\hline \multirow[t]{3}{*}{$16(705)$} & $10(2)$ & 42 & 557.9259 & 3 \\
\hline & & 50 & 444.9027 & 3 \\
\hline & & 40 & 457.7721 & 2 \\
\hline \multirow[t]{4}{*}{55 (705) } & $36(27)$ & 123 & 718.9610 & 2 \\
\hline & & 74 & 654.8900 & 2 \\
\hline & & 72 & 512.3270 & 3 \\
\hline & & 106 & 980.6179 & \\
\hline
\end{tabular}




\begin{tabular}{|c|c|c|c|c|}
\hline & & 90 & 821.5090 & 2 \\
\hline & & 73 & 557.9578 & 3 \\
\hline & & 59 & 518.3461 & 2 \\
\hline & & 80 & 848.4218 & 2 \\
\hline & & 52 & 612.3258 & 2 \\
\hline & & 51 & 534.3115 & 2 \\
\hline & & 49 & 601.3806 & 2 \\
\hline & & 68 & 594.4100 & 2 \\
\hline & & 86 & 551.6925 & 3 \\
\hline & & 45 & 666.4261 & 2 \\
\hline & & 82 & 1275.6824 & 2 \\
\hline & & 76 & 630.3881 & 3 \\
\hline & & 93 & 922.0209 & 2 \\
\hline & & 65 & 683.4185 & 3 \\
\hline & & 105 & 873.9819 & 2 \\
\hline & & 50 & 529.3714 & 2 \\
\hline & & 75 & 1000.4611 & 2 \\
\hline $18(705)$ & $10(3)$ & 105 & 942.9947 & 3 \\
\hline & & 64 & 654.9485 & 2 \\
\hline & & 41 & 551.7212 & 3 \\
\hline $14(67)$ & $1(0)$ & 27 & 537.8743 & 2 \\
\hline $24(705)$ & $14(8)$ & 88 & 942.9991 & 3 \\
\hline & & 63 & 654.9475 & 2 \\
\hline & & 40 & 512.3864 & 3 \\
\hline & & 100 & 980.6764 & 2 \\
\hline & & 44 & 612.4371 & 2 \\
\hline & & 50 & 521.3881 & 2 \\
\hline & & 45 & 551.7217 & 3 \\
\hline & & 69 & 683.5005 & 3 \\
\hline $33(705)$ & $19(11)$ & 88 & 942.8249 & 3 \\
\hline & & 63 & 654.8801 & 2 \\
\hline & & 46 & 512.2930 & 3 \\
\hline & & 84 & 980.4944 & 2 \\
\hline & & 77 & 821.4990 & 2 \\
\hline & & 41 & 557.9602 & 3 \\
\hline & & 64 & 612.3928 & 2 \\
\hline & & 48 & 534.2573 & 2 \\
\hline & & 40 & 601.3405 & 2 \\
\hline & & 46 & 551.6753 & 3 \\
\hline & & 49 & 529.2990 & 2 \\
\hline & & 91 & 1000.5303 & 2 \\
\hline $41(615)$ & $24(15)$ & 60 & 643.8221 & 2 \\
\hline & & 80 & 764.4039 & 2 \\
\hline & & 87 & 614.3680 & 2 \\
\hline & & 76 & 1038.8245 & 3 \\
\hline & & 72 & 499.2738 & 3 \\
\hline & & 44 & 432.7310 & 2 \\
\hline & & 91 & 874.3745 & 2 \\
\hline & & 51 & 514.9452 & 3 \\
\hline & & 70 & 843.4622 & 2 \\
\hline
\end{tabular}




\begin{tabular}{|c|c|c|c|c|}
\hline & & 95 & 779.4146 & 2 \\
\hline & & 51 & 461.5616 & 3 \\
\hline & & 72 & 973.4384 & 2 \\
\hline & & 44 & 413.7910 & 2 \\
\hline & & 70 & 634.8116 & 2 \\
\hline & & 92 & 746.6886 & 3 \\
\hline $26(615)$ & $15(5)$ & 81 & 614.3690 & 2 \\
\hline & & 51 & 499.2816 & 3 \\
\hline & & 50 & 432.7254 & 2 \\
\hline & & 50 & 509.6121 & 3 \\
\hline 30(615) & 18 (15) & 91 & 756.4052 & 2 \\
\hline & & 79 & 614.3667 & 2 \\
\hline & & 64 & 1038.8285 & 3 \\
\hline & & 75 & 748.4022 & 2 \\
\hline & & 42 & 432.7279 & 2 \\
\hline & & 46 & 514.9458 & 3 \\
\hline & & 44 & 562.9766 & 3 \\
\hline & & 57 & 605.3437 & 3 \\
\hline & & 70 & 965.4434 & 2 \\
\hline & & 44 & 413.7941 & 2 \\
\hline & & 79 & 634.8135 & 2 \\
\hline $36(615)$ & $18(8)$ & 94 & 756.5455 & 2 \\
\hline & & 42 & 614.4816 & 2 \\
\hline & & 42 & 1038.6868 & 3 \\
\hline & & 77 & 748.5637 & 2 \\
\hline & & 62 & 974.6602 & 3 \\
\hline & & 107 & 779.5539 & 2 \\
\hline & & 60 & 973.6480 & 2 \\
\hline $24(705)$ & $13(8)$ & 60 & 512.2892 & 3 \\
\hline & & 56 & 980.4840 & 2 \\
\hline & & 57 & 557.9355 & 3 \\
\hline & & 49 & 529.3002 & 2 \\
\hline & & 57 & 1000.4597 & 2 \\
\hline $13(615)$ & 7 (5) & 49 & 432.7277 & 2 \\
\hline & & 49 & 965.4771 & 2 \\
\hline $23(615)$ & $14(6)$ & 83 & 756.4122 & 2 \\
\hline & & 45 & 597.7695 & 2 \\
\hline & & 57 & 965.4379 & 2 \\
\hline 20(615) & $12(7)$ & 40 & 643.8247 & 2 \\
\hline & & 70 & 614.3732 & 2 \\
\hline & & 62 & 597.7711 & 2 \\
\hline & & 51 & 461.5615 & 3 \\
\hline & & 57 & 965.4409 & 2 \\
\hline & & 47 & 634.8518 & 2 \\
\hline 10(705) & $6(3)$ & 67 & 512.2888 & 3 \\
\hline & & 50 & 534.2579 & 2 \\
\hline $6(530)$ & $3(3)$ & 64 & 680.3580 & 2 \\
\hline & & 52 & 724.4147 & 2 \\
\hline
\end{tabular}




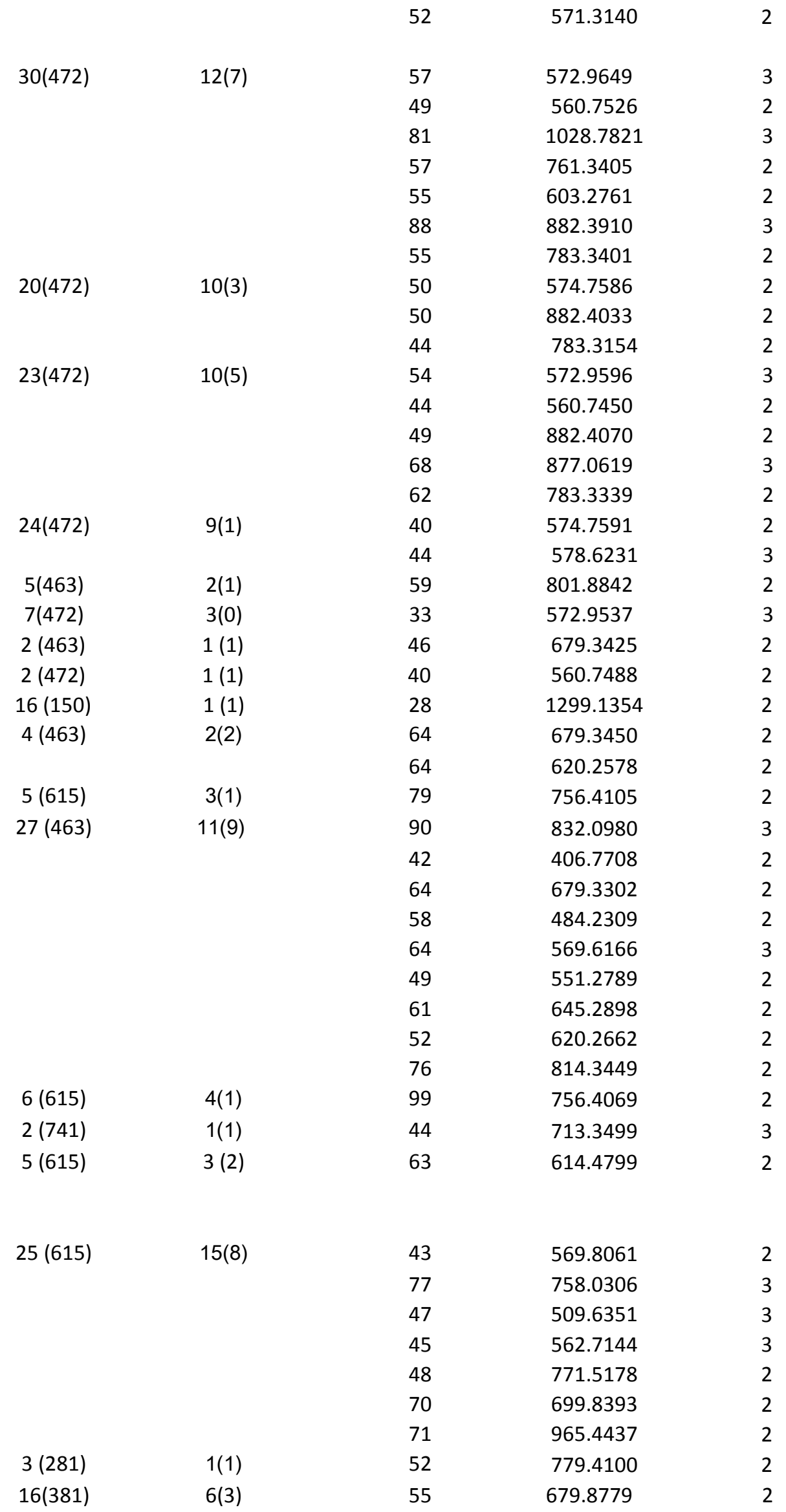




\begin{tabular}{|c|c|c|c|c|}
\hline & & 78 & 779.4035 & 2 \\
\hline & & 62 & 659.8059 & 2 \\
\hline \multirow[t]{5}{*}{$29(381)$} & $11(3)$ & 40 & 616.8057 & 2 \\
\hline & & 41 & 679.8832 & 2 \\
\hline & & 50 & 779.4092 & 2 \\
\hline & & 48 & 424.7524 & 2 \\
\hline & & 128 & 982.9702 & 2 \\
\hline \multirow[t]{19}{*}{$66(434)$} & $24(17)$ & 78 & 725.3635 & 2 \\
\hline & & 141 & 902.9760 & 2 \\
\hline & & 40 & 472.2608 & 3 \\
\hline & & 64 & 640.7977 & 2 \\
\hline & & 41 & 760.4204 & 2 \\
\hline & & 93 & 756.1602 & 4 \\
\hline & & 52 & 572.3150 & 2 \\
\hline & & 70 & 980.9689 & 2 \\
\hline & & 48 & 408.2486 & 2 \\
\hline & & 80 & 786.8836 & 2 \\
\hline & & 64 & 550.7736 & 2 \\
\hline & & 74 & 618.9609 & 3 \\
\hline & & 46 & 1198.0494 & 2 \\
\hline & & 74 & 730.3997 & 3 \\
\hline & & 40 & 504.2598 & 2 \\
\hline & & 88 & 817.4158 & 2 \\
\hline & & 74 & 514.5961 & 3 \\
\hline & & 61 & 1177.0785 & 2 \\
\hline & & 45 & 452.7353 & 2 \\
\hline \multirow[t]{6}{*}{$16(434)$} & $7(6)$ & 141 & 902.9760 & 2 \\
\hline & & 41 & 760.4204 & 2 \\
\hline & & 52 & 572.3150 & 2 \\
\hline & & 64 & 550.7736 & 2 \\
\hline & & 74 & 618.9609 & 3 \\
\hline & & 57 & 533.6057 & 3 \\
\hline \multirow[t]{3}{*}{$14(434)$} & $4(3)$ & 41 & 760.4204 & 2 \\
\hline & & 88 & 817.4158 & 2 \\
\hline & & 61 & 1177.0785 & 2 \\
\hline \multirow[t]{2}{*}{$16(417)$} & $7(2)$ & 87 & 545.6066 & 3 \\
\hline & & 57 & 666.8726 & 2 \\
\hline \multirow[t]{8}{*}{$50(334)$} & $15(12)$ & 64 & 502.2704 & 3 \\
\hline & & 50 & 576.3064 & 2 \\
\hline & & 84 & 878.4780 & 2 \\
\hline & & 60 & 569.6281 & 3 \\
\hline & & 54 & 466.7564 & 2 \\
\hline & & 68 & 970.5182 & 3 \\
\hline & & 42 & 588.8204 & 2 \\
\hline & & 74 & 697.3663 & 2 \\
\hline
\end{tabular}




\begin{tabular}{|c|c|c|c|c|}
\hline \multirow[t]{11}{*}{47 (333) } & $14(11)$ & 74 & 727.9177 & 2 \\
\hline & & 74 & 844.9247 & 2 \\
\hline & & 63 & 668.6491 & 3 \\
\hline & & 78 & 498.9350 & 3 \\
\hline & & 48 & 457.8002 & 2 \\
\hline & & 104 & 631.8160 & 2 \\
\hline & & 72 & 646.8474 & 2 \\
\hline & & 45 & 485.2909 & 2 \\
\hline & & 61 & 579.8231 & 2 \\
\hline & & 54 & 643.8713 & 2 \\
\hline & & 62 & 574.2930 & 2 \\
\hline \multirow[t]{3}{*}{30 (333) } & $8(3)$ & 87 & 844.9230 & 2 \\
\hline & & 106 & 631.8107 & 2 \\
\hline & & 63 & 646.8494 & 2 \\
\hline \multirow[t]{5}{*}{$51(254)$} & $8(5)$ & 57 & 890.3876 & 2 \\
\hline & & 61 & 537.2911 & 2 \\
\hline & & 79 & 813.7306 & 3 \\
\hline & & 46 & 575.8348 & 2 \\
\hline & & 51 & 1025.1666 & 3 \\
\hline \multirow[t]{9}{*}{$64(248)$} & $11(9)$ & 64 & 676.8853 & 2 \\
\hline & & 64 & 562.2825 & 2 \\
\hline & & 59 & 669.3477 & 2 \\
\hline & & 43 & 508.6035 & 3 \\
\hline & & 72 & 729.8674 & 2 \\
\hline & & 96 & 801.9479 & 2 \\
\hline & & 42 & 489.5818 & 3 \\
\hline & & 79 & 629.3113 & 2 \\
\hline & & 76 & 1023.8698 & 3 \\
\hline \multirow[t]{3}{*}{10 (705) } & $5(3)$ & 42 & 942.9452 & 3 \\
\hline & & 78 & 654.8294 & 2 \\
\hline & & 75 & 821.4211 & 2 \\
\hline $17(67)$ & $1(0)$ & 34 & 465.8785 & 3 \\
\hline $7(161)$ & $1(0)$ & 24 & 614.8198 & 2 \\
\hline \multirow[t]{5}{*}{$68(150)$} & $7(5)$ & 132 & 748.3681 & 2 \\
\hline & & 89 & 684.3212 & 2 \\
\hline & & 126 & 872.0895 & 3 \\
\hline & & 70 & 631.2913 & 2 \\
\hline & & 83 & 830.4034 & 3 \\
\hline \multirow[t]{8}{*}{$43(264)$} & $11(7)$ & 61 & 661.8372 & 2 \\
\hline & & 91 & 687.3716 & 2 \\
\hline & & 40 & 499.7993 & 2 \\
\hline & & 44 & 493.2710 & 2 \\
\hline & & 61 & 589.3012 & 2 \\
\hline & & 58 & 553.2901 & 2 \\
\hline & & 47 & 568.7920 & 2 \\
\hline & & 40 & 524.7878 & 2 \\
\hline
\end{tabular}




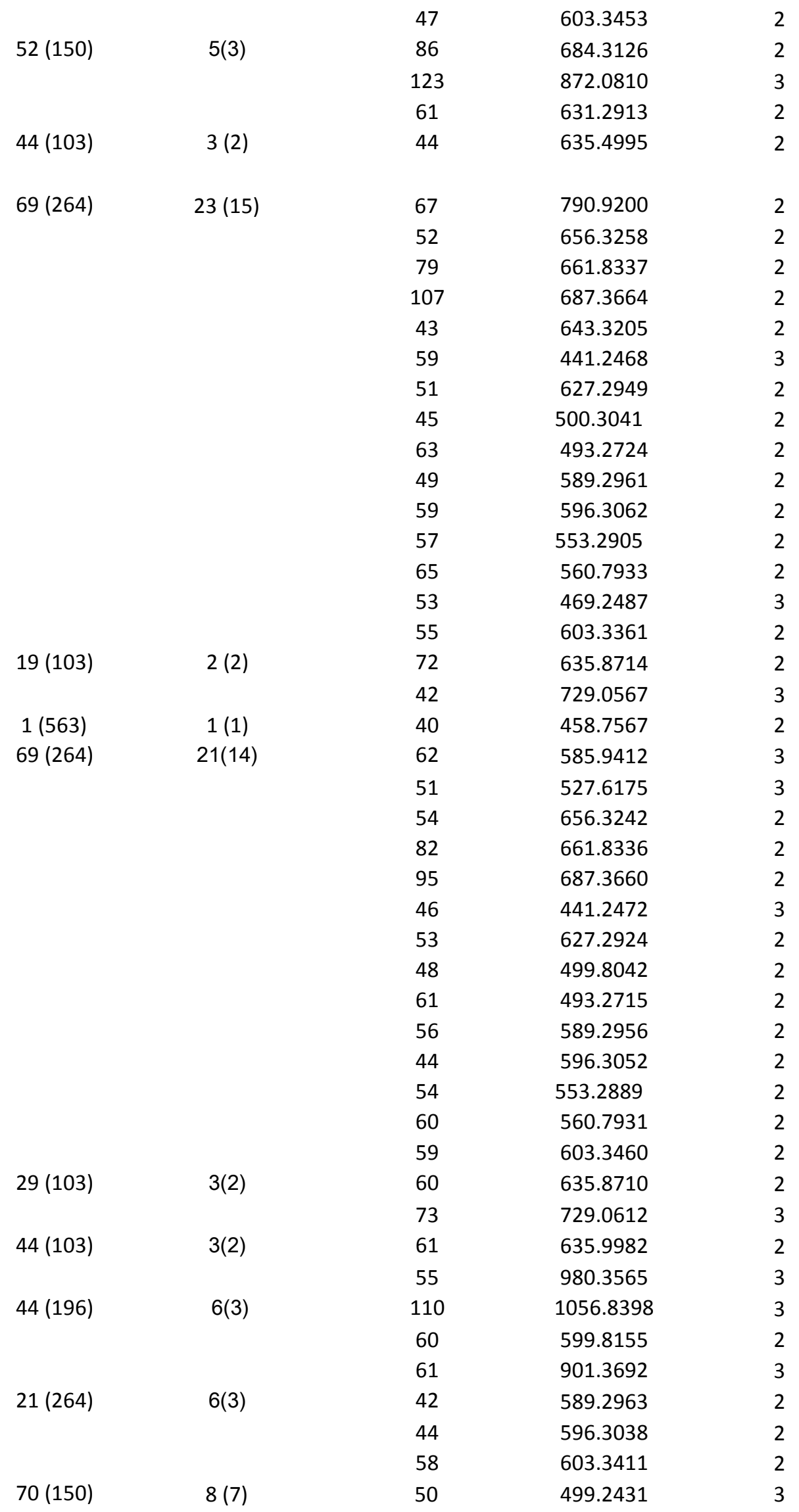




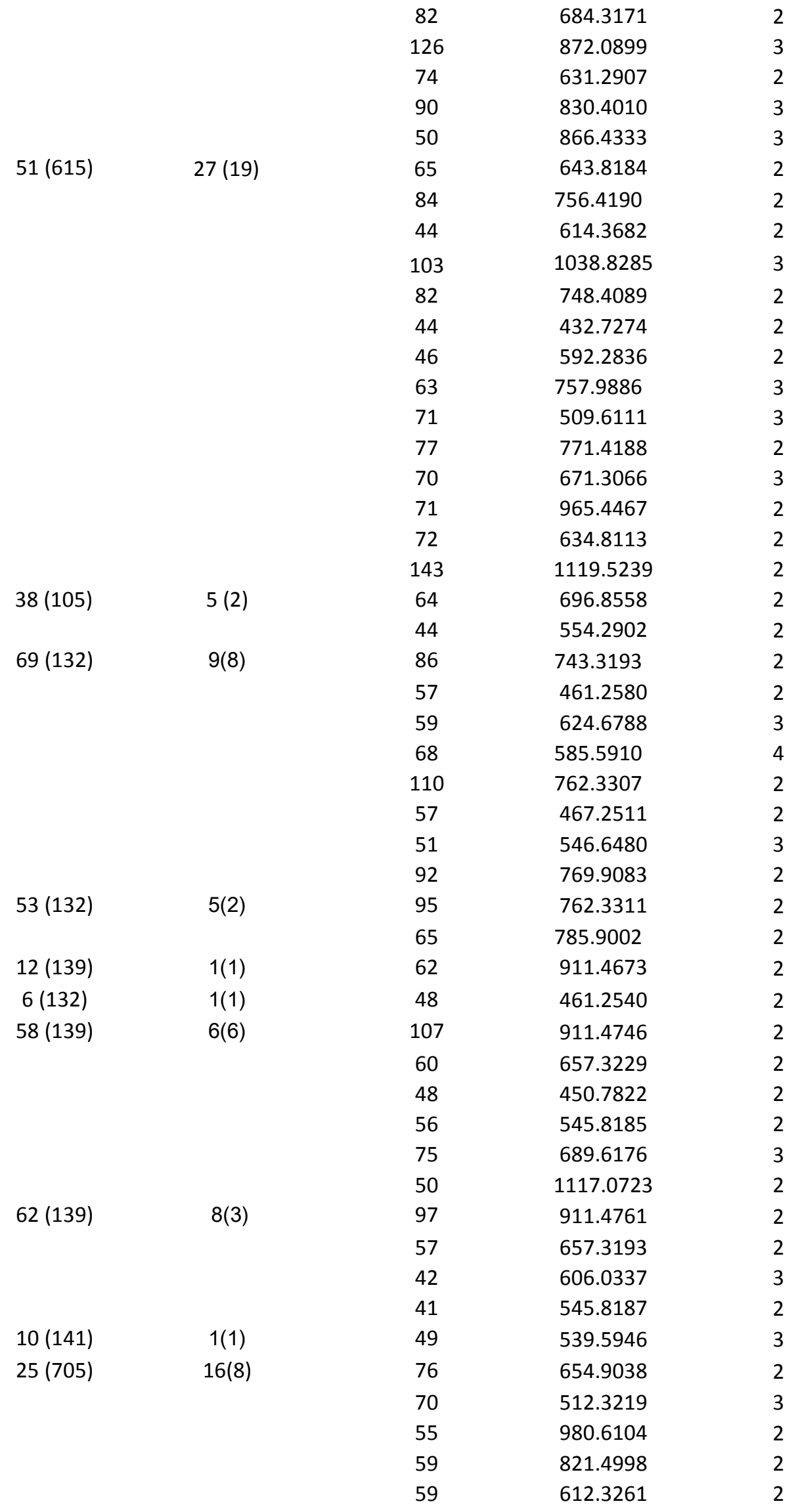




$\begin{array}{lcccc} & & 41 & 457.2418 & 2 \\ 47(147) & 45 & 529.3430 & 2 \\ & & 59 & 1008.4685 & 2 \\ & 5(5) & 46 & 437.7079 & 2 \\ & & 52 & 663.3895 & 2 \\ 3(409) & & 76 & 714.8303 & 2 \\ 92(55) & 128 & 877.5216 & 2 \\ & & 67 & 1254.6383 & 2 \\ & 5(1) & 61 & 691.9245 & 2 \\ & & 46 & 444.2158 & 2 \\ 1(1079) & & 50 & 494.2502 & 4 \\ 41(55) & 1(0) & 55 & 599.7548 & 2 \\ 3(705) & 1(1) & 64 & 1028.9528 & 2 \\ 32(67) & 2(0) & 88 & 934.7783 & 3 \\ 35(68) & & 38 & 658.8801 & 2 \\ & 2(1) & 90 & 934.7844 & 3 \\ & 1(1) & 39 & 551.6224 & 3 \\ & & & & \\ & & 63 & 538.3372 & 2 \\ & & 92 & 859.7986 & 3\end{array}$




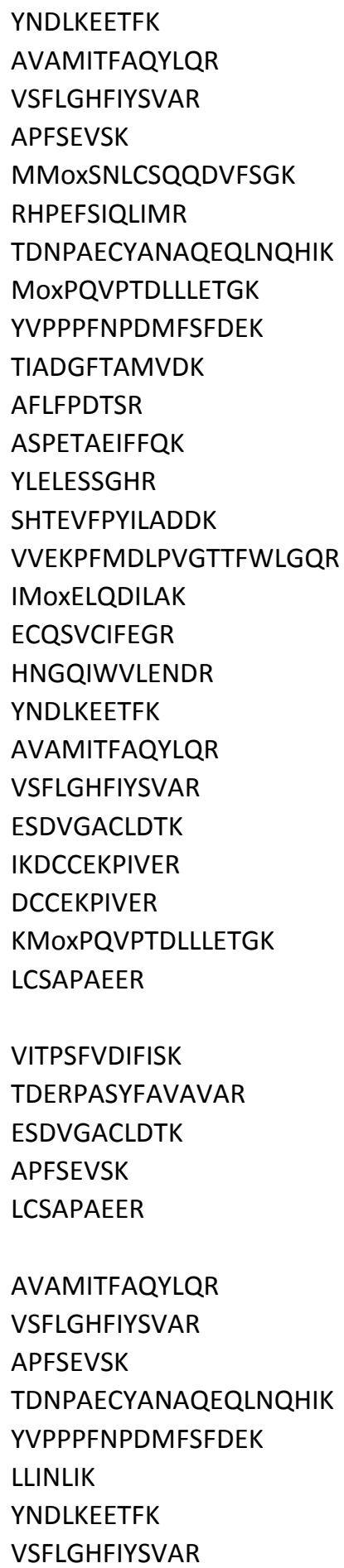




\begin{tabular}{|c|c|}
\hline ESDVGACLDTK & $199-209$ \\
\hline APFSEVSK & $254-261$ \\
\hline LCSAPAEER & $541-549$ \\
\hline YNDLKEETFK & $38-47$ \\
\hline AVAMITFAQYLQR & $48-60$ \\
\hline LVKDVVDLAQK & $69-79$ \\
\hline VSFLGHFIYSVAR & $160-172$ \\
\hline MoxMoxSNLCSQQDVFSGK & $288-302$ \\
\hline IKDCCEKPIVER & $303-314$ \\
\hline TDNPAECYANAQEQLNQHIK & $391-410$ \\
\hline KMoxPQVPTDLLLETGK & $442-456$ \\
\hline LLINLIK & $556-562$ \\
\hline TIADGFTAMoxVDK & $574-585$ \\
\hline YNDLKEETFK & $38-47$ \\
\hline AVAMoxITFAQYLQR & $48-60$ \\
\hline VSFLGHFIYSVAR & $160-172$ \\
\hline IKDCCEKPIVER & 303-314 \\
\hline SFEAGHDAFMAEFVYEYSR & $346-364$ \\
\hline RHPEFSIQLIMR & $365-376$ \\
\hline TDNPAECYANAQEQLNQHIK & $391-410$ \\
\hline MPQVPTDLLLETGK & $443-456$ \\
\hline MPQVPTDLLLETGKK & $443-457$ \\
\hline YVPPPFNPDMoxFSFDEK & $525-540$ \\
\hline LCSAPAEER & $541-549$ \\
\hline LLINLIK & $556-562$ \\
\hline TIADGFTAMoxVDK & $574-585$ \\
\hline HPNIICDCCKK & $86-96$ \\
\hline TDSQDMoxNEDT & $93-102$ \\
\hline APFSEVSK & $254-261$ \\
\hline GTEFTVNDLQGK & $120-131$ \\
\hline FFSASCVPGATIEQK & $174-188$ \\
\hline NAPYSGYSGAFHCLK & 204-218 \\
\hline AQSDFGVDTK & $289-298$ \\
\hline SDFHLFGPPGK & 299-309 \\
\hline TDERPASYFAVAVAR & $443-457$ \\
\hline FYTVISSLK & $680-688$ \\
\hline YNDLKEETFK & $38-47$ \\
\hline TNCDLLHDHGEADFLK & $418-433$ \\
\hline SDFHLFGPPGK & 299-309 \\
\hline GTEFTVNDLQGK & $120-131$ \\
\hline SAGWNIPIGTLLHR & $141-154$ \\
\hline VEDIWSFLSK & $279-288$ \\
\hline SDFHLFGPPGK & 299-309 \\
\hline TDERPASYFAVAVAR & $443-457$ \\
\hline
\end{tabular}




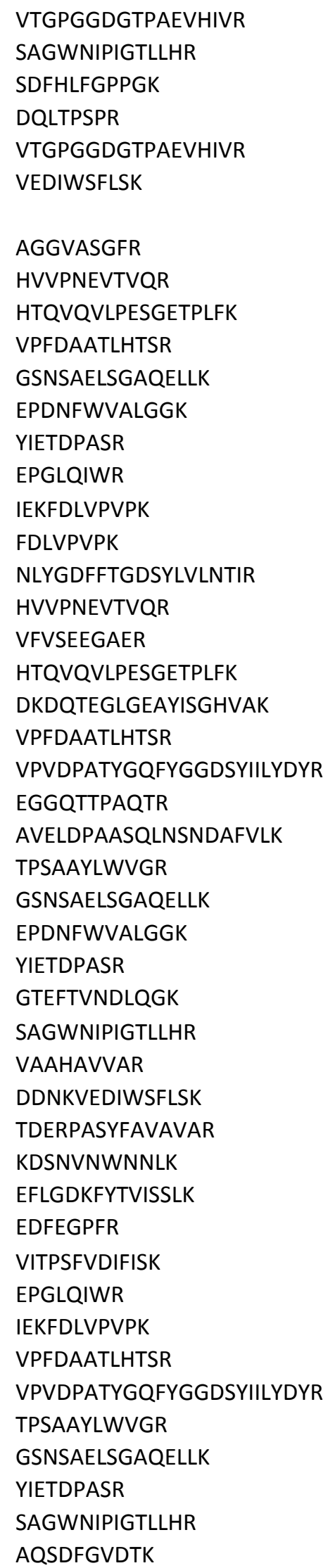

982-998

141-154

299-309

353-360

982-998

279-288

165-173

174-184

370-386

416-427

605-619

633-644

726-734

61-68

69-79

72-79

80-98

174-184

254-263

370-386

394-412

416-427

454-477

550-560

574-593

594-604

605-619

633-644

726-734

120-131

141-154

266-274

275-288

443-457

458-468

674-688

122-129

219-231

61-68

69-79

416-427

454-477

594-604

605-619

726-734

141-154

289-298 


\begin{tabular}{|c|c|}
\hline KGTEFTVNDLQGK & $119-131$ \\
\hline GTEFTVNDLQGK & $120-131$ \\
\hline SAGWNIPIGTLLHR & 141-154 \\
\hline FFSASCVPGATIEQK & $174-188$ \\
\hline AQSDFGVDTK & $289-298$ \\
\hline SDFHLFGPPGK & 299-309 \\
\hline TDERPASYFAVAVAR & $443-457$ \\
\hline NLQMoxDDFELLCTDGR & $579-593$ \\
\hline ECNLAEVPTHAVVVRPEK & $602-619$ \\
\hline FYTVISSLK & $680-688$ \\
\hline TCNPSDILQMCSFLEGK & $689-705$ \\
\hline HTQVQVLPESGETPLFK & $370-386$ \\
\hline VPFDAATLHTSR & $416-427$ \\
\hline VPVDPATYGQFYGGDSYIILYDYR & $454-477$ \\
\hline TPSAAYLWVGR & $594-604$ \\
\hline KGTEFTVNDLQGK & $119-131$ \\
\hline GTEFTVNDLQGK & $120-131$ \\
\hline SAGWNIPIGTLLHR & 141-154 \\
\hline GAIEWEGIESGSVEQAVAK & $155-173$ \\
\hline FFSASCVPGATIEQK & $174-188$ \\
\hline NAPYSGYSGAFHCLK & $204-218$ \\
\hline VAAHAVVAR & $266-274$ \\
\hline DDNKVEDIWSFLSK & $275-288$ \\
\hline VEDIWSFLSK & $279-288$ \\
\hline AQSDFGVDTK & $289-298$ \\
\hline SDFHLFGPPGK & 299-309 \\
\hline VPSLMoxDSQLYLGFEYYSAIQSMR & $329-351$ \\
\hline TDERPASYFAVAVAR & $443-457$ \\
\hline NLQMoxDDFELLCTDGR & $579-593$ \\
\hline ECNLAEVPTHAVVVRPEK & $602-619$ \\
\hline FYTVISSLK & $680-688$ \\
\hline TCNPSDILQMCSFLEGK & $689-705$ \\
\hline LKPIAAEVYEHTEGSTTSYYAVAVVK & $93-118$ \\
\hline KGTEFTVNDLQGK & $119-131$ \\
\hline GTEFTVNDLQGK & $120-131$ \\
\hline SAGWNIPIGTLLHR & 141-154 \\
\hline GAIEWEGIESGSVEQAVAK & $155-173$ \\
\hline FFSASCVPGATIEQK & $174-188$ \\
\hline DGKGDVAFVK & $219-228$ \\
\hline HTTVNENAPDQK & $229-240$ \\
\hline DDNKVEDIWSFLSK & $275-288$ \\
\hline VEDIWSFLSK & $279-288$ \\
\hline AQSDFGVDTK & $289-298$ \\
\hline SDFHLFGPPGK & 299-309 \\
\hline SDFHLFGPPGKK & $299-310$ \\
\hline KDQLTPSPR & $352-360$ \\
\hline TDERPASYFAVAVAR & $443-456$ \\
\hline DSNVNWNNLK & $459-468$ \\
\hline TGTCNFDEYFSEGCAPGSPPNSR & $494-516$ \\
\hline
\end{tabular}




\begin{tabular}{|c|c|}
\hline GDVAFIQHSTVEENTGGK & $554-571$ \\
\hline NLQMDDFELLCTDGR & $579-593$ \\
\hline ECNLAEVPTHAVVVRPEK & $602-619$ \\
\hline EFLGDKFYTVISSLK & $674-688$ \\
\hline FYTVISSLK & $680-688$ \\
\hline TCNPSDILQMCSFLEGK & $689-705$ \\
\hline KGTEFTVNDLQGK & 119-131 \\
\hline GTEFTVNDLQGK & $120-131$ \\
\hline SAGWNIPIGTLLHR & 141-154 \\
\hline GAIEWEGIESGSVEQAVAK & $155-173$ \\
\hline FFSASCVPGATIEQK & $174-188$ \\
\hline NAPYSGYSGAFHCLK & $204-218$ \\
\hline DGKGDVAFVK & $219-228$ \\
\hline DDNKVEDIWSFLSK & $275-288$ \\
\hline VEDIWSFLSK & $279-288$ \\
\hline AQSDFGVDTK & 289-298 \\
\hline SDFHLFGPPGK & 299-309 \\
\hline SDFHLFGPPGKK & 299-310 \\
\hline SDFHLFGPPGKKDPVLK & 299-315 \\
\hline DLLFKDSAIMLK & $316-327$ \\
\hline KDQLTPSPR & $352-360$ \\
\hline TDERPASYFAVAVAR & $443-457$ \\
\hline GDVAFIQHSTVEENTGGK & $554-571$ \\
\hline NLQMDDFELLCTDGR & $579-593$ \\
\hline ECNLAEVPTHAVVVRPEK & $602-619$ \\
\hline EGTTYKEFLGDKFYTVISSLK & $668-688$ \\
\hline EFLGDKFYTVISSLK & $674-688$ \\
\hline FYTVISSLK & $680-688$ \\
\hline TCNPSDILQMCSFLEGK & $689-705$ \\
\hline LKPIAAEVYEHTEGSTTSYYAVAVVK & $93-118$ \\
\hline KGTEFTVNDLQGK & 119-131 \\
\hline GTEFTVNDLQGK & $120-131$ \\
\hline SAGWNIPIGTLLHR & 141-154 \\
\hline GAIEWEGIESGSVEQAVAK & $155-173$ \\
\hline FFSASCVPGATIEQK & $174-188$ \\
\hline NAPYSGYSGAFHCLK & 204-218 \\
\hline VAAHAVVAR & $266-274$ \\
\hline DDNKVEDIWSFLSK & $275-288$ \\
\hline VEDIWSFLSK & $279-288$ \\
\hline AQSDFGVDTK & $289-298$ \\
\hline SDFHLFGPPGK & 299-309 \\
\hline GEADAVALDGGLVYTAGVCGLVPVMAER & 406-433 \\
\hline TDERPASYFAVAVAR & $443-457$ \\
\hline DSNVNWNNLK & $459-468$ \\
\hline ECNLAEVPTHAVVVRPEK & $602-619$ \\
\hline EFLGDKFYTVISSLK & $674-688$ \\
\hline FYTVISSLK & $680-688$ \\
\hline TCNPSDILQMCSFLEGK & $689-705$ \\
\hline LKPIAAEVYEHTEGSTTSYYAVAVVK & $93-118$ \\
\hline KGTEFTVNDLQGK & 119-131 \\
\hline
\end{tabular}




\begin{tabular}{|c|c|}
\hline GTEFTVNDLQGK & $120-131$ \\
\hline SAGWNIPIGTLLHR & $141-154$ \\
\hline FFSASCVPGATIEQK & $174-188$ \\
\hline VAAHAVVAR & $266-274$ \\
\hline DDNKVEDIWSFLSK & $275-288$ \\
\hline VEDIWSFLSK & $279-288$ \\
\hline AQSDFGVDTK & 289-298 \\
\hline SDFHLFGPPGK & 299-309 \\
\hline GEADAVALDGGLVYTAGVCGLVPVMAER & $406-433$ \\
\hline TDERPASYFAVAVAR & $443-457$ \\
\hline TGTCNFDEYFSEGCAPGSPPNSR & $494-516$ \\
\hline FYTVISSLK & $680-688$ \\
\hline TCNPSDILQMCSFLEGK & $689-705$ \\
\hline GTEFTVNDLQGK & $120-131$ \\
\hline YNDLKEETFK & $38-47$ \\
\hline AVAMITFAQYLQR & $48-60$ \\
\hline VSFLGHFIYSVAR & $160-172$ \\
\hline MPQVPTDLLLETGK & $443-456$ \\
\hline RPCFTAMGVDTK & $513-524$ \\
\hline YVPPPFNPDMFSFDEK & $525-540$ \\
\hline APFSEVSK & $254-261$ \\
\hline SQCIMoxEAEFDEKPADLPSLVEK & $315-336$ \\
\hline TDNPAECYANAQEQLNQHIK & $391-410$ \\
\hline YVPPPFNPDMFSFDEK & $525-540$ \\
\hline LLINLIK & $556-562$ \\
\hline KPQMTEEQIK & $564-573$ \\
\hline YNDLKEETFK & $38-47$ \\
\hline AVAMITFAQYLQR & $48-60$ \\
\hline VSFLGHFIYSVAR & $160-172$ \\
\hline ESDVGACLDTK & 199-209 \\
\hline FVHDSIGVHK & $262-271$ \\
\hline TDNPAECYANAQEQLNQHIK & $391-410$ \\
\hline MPQVPTDLLLETGK & $443-456$ \\
\hline YVPPPFNPDMFSFDEK & $525-540$ \\
\hline LLINLIK & $556-562$ \\
\hline HVMIDCSPYLQVVR & $89-102$ \\
\hline DGNTMOxVACPR & $103-112$ \\
\hline ILSPVCGTDGFTYDNECGICAHNAEQR & 179-205 \\
\hline EEVPELDCSK & $349-358$ \\
\hline HVMoxIDCSPYLQVVR & $89-102$ \\
\hline DGNTMVACPR & $103-112$ \\
\hline EEVPELDCSK & $349-358$ \\
\hline DGTSWVACPR & $38-47$ \\
\hline DGNTMoxVACPR & $103-112$ \\
\hline LEIGSVDCSK & $152-161$ \\
\hline TLVACPR & $172-178$ \\
\hline DNLDNNIPSSLR & $159-170$ \\
\hline AVAMITFAQYLQR & $48-60$ \\
\hline
\end{tabular}




\begin{tabular}{|c|c|}
\hline LVKDVVDLAQK & $69-79$ \\
\hline VSFLGHFIYSVAR & $160-172$ \\
\hline FVHDSIGVHK & $262-271$ \\
\hline MoxMoxSNLCSQQDVFSGK & $288-302$ \\
\hline TDNPAECYANAQEQLNQHIK & $391-410$ \\
\hline YVPPPFNPDMFSFDEK & $525-540$ \\
\hline TIADGFTAMoxVDK & $574-585$ \\
\hline SAGWNIPIGTLLHR & $141-154$ \\
\hline GAIEWEGIESGSVEQAVAK & $155-173$ \\
\hline TDERPASYFAVAVAR & $443-457$ \\
\hline ECNLAEVPTHAVVVRPEK & $602-619$ \\
\hline IRDLLER & $623-629$ \\
\hline EFLGDKFYTVISSLK & $674-688$ \\
\hline FYTVISSLK & $680-688$ \\
\hline TCNPSDILQMCSFLEGK & $689-705$ \\
\hline AVAMITFAQYLQR & $48-60$ \\
\hline VSFLGHFIYSVAR & $160-172$ \\
\hline APFSEVSK & $254-261$ \\
\hline YVPPPFNPDMFSFDEK & $525-540$ \\
\hline KGTEFTVNDLQGK & $119-131$ \\
\hline GTEFTVNDLQGK & $120-131$ \\
\hline TSCHTGLGR & $132-140$ \\
\hline SAGWNIPIGTLLHR & $141-154$ \\
\hline GAIEWEGIESGSVEQAVAK & $155-173$ \\
\hline SDFHLFGPPGKK & $299-310$ \\
\hline TDERPASYFAVAVAR & $443-457$ \\
\hline NLQMoxDDFELLCTDGR & $579-593$ \\
\hline ECNLAEVPTHAVVVRPEK & $602-619$ \\
\hline FYTVISSLK & $680-688$ \\
\hline TCNPSDILQMCSFLEGK & $689-705$ \\
\hline VSFLGHFIYSVAR & $160-172$ \\
\hline LLINLIK & $556-562$ \\
\hline LDIDSEPTIAR & $32-42$ \\
\hline NTGIICTIGPASR & $43-55$ \\
\hline GVNLPGAAVDLPAVSEK & $207-223$ \\
\hline FGVEQNVDMoxVFASFIR & $230-245$ \\
\hline GDYPLEAVR & $367-375$ \\
\hline AVAMITFAQYLQR & $48-60$ \\
\hline VSFLGHFIYSVAR & $160-172$ \\
\hline IYVDDGLISLLVK & $173-185$ \\
\hline DNLDNNIPSSLR & $159-170$ \\
\hline SPCVASCNIPVVSGR & $199-213$ \\
\hline ECEDIYR & $214-220$ \\
\hline YQLSVSNYK & $341-349$ \\
\hline GDLGIEIPAEK & 294-304 \\
\hline APFSEVSK & $254-261$ \\
\hline RHPEFSIQLIMR & $365-376$ \\
\hline HPEFSIQLIMoxR & $366-376$ \\
\hline TDNPAECYANAQEQLNQHIK & $391-410$ \\
\hline KMoxPQVPTDLLLETGK.K & $442-456$ \\
\hline
\end{tabular}




\begin{tabular}{|c|c|}
\hline MoxPQVPTDLLLETGK & $443-456$ \\
\hline MACSEGYLSIVIHDTCR & $474-490$ \\
\hline YVPPPFNPDMFSFDEK & $525-540$ \\
\hline LLINLIK & $556-562$ \\
\hline TIADGFTAMVDK & $574-585$ \\
\hline QSDINTCFGEEGANLIVQSR & $589-608$ \\
\hline HIADLAGNPEVILPVPAFNVINGGSHAGNK & $133-162$ \\
\hline APFSEVSK & $254-261$ \\
\hline IKDCCEKPIVER & $303-314$ \\
\hline RHPEFSIQLIMR & $365-376$ \\
\hline KMoxPQVPTDLLLETGK & $442-456$ \\
\hline MPQVPTDLLLETGK & $443-456$ \\
\hline MACSEGYLSIVIHDTCR & $474-490$ \\
\hline RPCFTAMoxGVDTK & $513-524$ \\
\hline YVPPPFNPDMFSFDEK & $525-540$ \\
\hline LLINLIK & $556-562$ \\
\hline IKDCCEKPIVER & $303-314$ \\
\hline TDNPAECYANAQEQLNQHIK & $391-410$ \\
\hline YVPPPFNPDMFSFDEK & $525-540$ \\
\hline ELFDPVIEDR & $87-96$ \\
\hline LSVEALGSLGGDLK & $157-170$ \\
\hline FCTGLTQIETLFK & $253-265$ \\
\hline ELFDPVIEDR & $87-96$ \\
\hline LSVEALGSLGGDLK & $157-170$ \\
\hline FGEVLKR & $308-314$ \\
\hline RGTGGVDTAAVGGVFDVSNADR & $320-341$ \\
\hline KVYIQDVCR & $59-67$ \\
\hline HLVLIAQEEGAR & $102-113$ \\
\hline FELGCYALCPSIK & $129-141$ \\
\hline MOXPEFYQR & $148-154$ \\
\hline APLDLYTK & $203-210$ \\
\hline SQQAVAGTVHVSVFK & $342-356$ \\
\hline AGACPPTFTISGQCHGGLLNCCAK & $41-64$ \\
\hline GGASIVTPR & $203-211$ \\
\hline GNPTVEVDLYTNK & $16-28$ \\
\hline AAVPSGASTGIYEALELR & $33-50$ \\
\hline NVNVVEQEKIDK & $81-92$ \\
\hline IDKLMoxLEMoxDGTENK & $90-103$ \\
\hline LMLEMDGTENK & $93-103$ \\
\hline FGANAILGVSLAVCK & $106-120$ \\
\hline HIADLAGNPEVILPVPAFNVINGGSHAGNK & $133-162$ \\
\hline IGAEVYHNLK & 184-193 \\
\hline DATNVGDEGGFAPNILENK & $203-221$ \\
\hline EALELLK & $222-228$ \\
\hline VVIGMDVAASEFYR & $240-253$ \\
\hline NYPVVSIEDPFDQDDWAAWKK & $286-306$ \\
\hline FTASVGIQVVGDDLTVTNPK & $307-326$ \\
\hline SCNCLLLK & $336-343$ \\
\hline
\end{tabular}


VNQIGSVTESLQACK

LAQSNGWGVMVSHR

IEEELGSK

KISVVEQEKIDK

FGANAILGVSLAVCK

VNQIGSVTESIQACK

LGDVYVNDAFGTAHR

ACANPANGSVILLENLR

LGDVYVNDAFGTAHR

ALESPERPFLAILGGAK

VLNNMOXQIGNSLFDEEGSK

ITLPVDFITADK

GCITIIGGGDTATCCAK

VSHVSTGGGASLELLEGK

IALGDDSPAIAQK

IVATTLTSPQLFAEWK

TDEGQPWVLPVVR

KVEQLIAGDGSLNHEYLPILGLPEFR

NFGLYNER

VGNLSVVGKDEDNVQR

IVATTLTSPQLFAEWK

VVVVGNPANTNCLIASK

FVEGLPINDFSR

ITVVGVGQVGMoxACAISILGK

GLCDELALVDVLEDK

IVADKDYAVTANSK

DYAVTANSK

IVVVTAGVR

VIGSGCNLDTAR

QVVESAYEVIR

LKDDEVAQLK

LKDDEVAQLKK

KSADTLWSIQK

DLADELTLVDVVEDK

VIGSGCNLDSAR

QVVDSAYEVIK

VEILRNAIRYIESLQALLR

KFFVGGNWK

SLGELIHTLNGAK

LSADTEVVCGAPSIYLDFAR

IGVAAQNCYK

GAFTGEISPAMoxIK

DIGAAWVILGHSER

HVFGESDELIGQK

VAHALAEGLGVIACIGEK
344-358

359-372

413-420

81-92

106-120

344-358

157-171

107-123

157-171

200-216

247-264

280-291

366-382

389-406

86-98

305-320

42-54

55-80

259-266

267-282

305-320

126-142

299-310

23-42

43-57

77-90

82-90

91-99

158-169

233-243

308-317

308-318

318-328

43-57

158-169

233-243

138-156

5-13

20-32

33-52

59-68

72-84

85-98

100-112

113-130 
VVLAYEPVWAIGTGK
TATPQQAQEVHEK
SHVSDAVAQSTR
ELASQHDVDGFLVGGASLKPEFVDIINAK
LGLDFPNLPYLIDGDVK
LTQSNAILR
LADNLDTLSAAAAK
LTPVAEEAR
LSADTEVVCGAPSIYLDFAR
GAFTGEISPAMOXIK
DIGAAWVILGHSER
HVFGESDELIGQK
VVLAYEPVWAIGTGK
TATPQQAQEVHEK
SHVSDAVAQSTR
IIYGGSVTGGNCK

GSPAANVAVKVFKK

KAADGTWQDFATGK

AADGTWQDFATGK

TTEFGEIHELTTEEQFVEGVYR

VEFDTSSYWK

GLGLSPFHEYADVVFTANDSGHR

HYTIAALLSPFSYSTTAVVSDPQE

DAIAQFESSAVGK

LADNLDTLSAAAAK

LISFLDELQK

VSFLGHFIYSVAR

VEFDTSSYWK

GTVTAFSPFDAR

VLTEILASR

TPAEVQNIK

ITGETSGHFQR

SVPAYFAETLYYSMK

SEIDLLDIR

DMOXVDVYLETVK

DAIAQFESSAVGK

LADNLDTLSAAAAK

IRPFLDQFSAK

LTPVAQELK

LTPVAEEAR

NLAPYSDELR

GIPQASEYQAK

MOXTPLVQEFR

LISFLDELQK

VAPTITLFPPSK

SFWQHDEPQTPLDR

DMOXVDVYLETVK

DAIAQFESSAVGK
160-174

175-187

194-205

219-247

53-69

70-78

69-82

164-172

33-52

72-84

85-98

100-112

160-174

175-187

194-205

206-218

45-58

58-71

59-71

72-93

94-103

104-126

127-150

51-63

69-82

252-261

160-172

94-103

7-18

109-117

118-126

141-151

246-260

277-285

$36-46$

51-63

69-82

120-130

142-150

164-172

185-194

208-218

230-238

252-261

4-15

20-33

36-46

51-63 


\begin{tabular}{|c|c|}
\hline LADNLDTLSAAAAK & $69-82$ \\
\hline IRPFLDQFSAK & $120-130$ \\
\hline WTEELEQYR & $131-139$ \\
\hline LTPVAEEAR & $164-172$ \\
\hline NLAPYSDELR & 185-194 \\
\hline GIPQASEYQAK & $208-218$ \\
\hline VMoxEQLSNLR & $219-227$ \\
\hline MTPLVQEFR & $230-238$ \\
\hline LISFLDELQK & $252-261$ \\
\hline VAPTITLFPPSK & $4-15$ \\
\hline YNDLKEETFK & $38-47$ \\
\hline AVAMITFAQYLQR & $48-60$ \\
\hline LVKDVVDLAQK & $69-79$ \\
\hline CVANEDAPECSKPLPSIILDEICQVEK & $80-106$ \\
\hline DSYGAMoxADCCSK & $109-120$ \\
\hline VSFLGHFIYSVAR & $160-172$ \\
\hline RHPFLYAPAILSFAVDFEHALQSCCK & $173-198$ \\
\hline HPFLYAPAILSFAVDFEHALQSCCK & 174-198 \\
\hline ESDVGACLDTK & $199-209$ \\
\hline VAPTITLFPPSK & $4-15$ \\
\hline VAPTITLFPPSK & $4-15$ \\
\hline VAPTITLFPPSKEELNEATK & $4-23$ \\
\hline ATLVCLINDFYPSPVTVDWVIDGSTR & $24-49$ \\
\hline DAIAQFESSAVGK & $51-63$ \\
\hline LADNLDTLSAAAAK & $69-82$ \\
\hline MoxTPLVQEFR & $230-238$ \\
\hline LISFLDELQK & $252-261$ \\
\hline DDTALNGIR & $66-74$ \\
\hline YNDLKEETFK & $38-47$ \\
\hline AVAMITFAQYLQR & $48-60$ \\
\hline CSYEGLSK & $61-68$ \\
\hline VSFLGHFIYSVAR & $160-172$ \\
\hline AVAMoxITFAQYLQR & $48-60$ \\
\hline ESDVGACLDTK & 199-209 \\
\hline YWGVASFLQK & $111-120$ \\
\hline ELNEDGTCADSYSFVFSR & $143-160$ \\
\hline QIDLCLDR & $177-184$ \\
\hline QIDLCLDRK & $177-185$ \\
\hline LISFLDELQK & $252-261$ \\
\hline GSPAANVAVKVFKK & $45-58$ \\
\hline AADGTWQDFATGK & $59-71$ \\
\hline TTEFGEIHELTTEEQFVEGVYR & $72-93$ \\
\hline VEFDTSSYWK & $94-103$ \\
\hline GLGLSPFHEYADVVFTANDSGHR & $104-126$ \\
\hline LADSHNFDEYMoxK & $11-22$ \\
\hline ALGVGFAMoxR & $23-31$ \\
\hline QVGNVTKPTVIISSEGDK & $32-49$ \\
\hline QVGNVTKPTVIISSEGDKVVIR & $32-53$ \\
\hline
\end{tabular}




\begin{tabular}{|c|c|}
\hline NTEISFK & $60-66$ \\
\hline LGEEFDETTPDDR & $67-79$ \\
\hline SVVTLDGDK & $83-91$ \\
\hline SVVTLDGDKLVHVQK & $83-97$ \\
\hline MVMTLTFGDVVAVR & $114-127$ \\
\hline AADGTWQDFATGK & $59-71$ \\
\hline LADSHNFDEYMK & $11-22$ \\
\hline ALGVGFAMoxR & $23-31$ \\
\hline QVGNVTKPTVIISSEGDKVVIR & $32-53$ \\
\hline LGEEFDETTPDDR & $67-79$ \\
\hline MoxVMoxTLTFGDVVAVR & $114-127$ \\
\hline LLGAPVPVDENDEGLQR & $30-46$ \\
\hline ALQFAMOXAEYNR & $47-57$ \\
\hline RQLVSGIK & $75-82$ \\
\hline YILQVEIGR & 83-91 \\
\hline SSGDLQSCEFHDEPEMOXAK & $97-114$ \\
\hline SIPPACDK & $1-8$ \\
\hline DYSPVCGTDGK & $18-28$ \\
\hline TYPNECVLCLSNSEENK & $29-45$ \\
\hline TYPNECVLCLSNSEENKNVQIYK & $29-51$ \\
\hline SIPPACDK & $1-8$ \\
\hline DYSPVCGTDGK & $18-28$ \\
\hline TYPNECVLCLSNSEENK & $29-45$ \\
\hline TYPNECVLCLSNSEENKNVQIYK & $29-51$ \\
\hline LKPIAAEVYEHTEGSTTSYYAVAVVK & $93-118$ \\
\hline APSVDIGTCR & $45-54$ \\
\hline AGACPPTFTISGQCHGGLLNCCAK & $41-64$ \\
\hline TQGNFCR & $34-40$ \\
\hline AGACPPTFTISGQCHGGLLNCCAK & $41-64$ \\
\hline AGACPPTFTISGQCHGGLLNCCAK & $41-64$ \\
\hline APSVDIGTCR & $45-54$ \\
\hline AGACPPTFTISGQCHGGLLNCCAK & $41-64$ \\
\hline SFEAGHDAFMAEFVYEYSR & $346-364$ \\
\hline SFEAGHDAFMAEFVYEYSR & $346-364$ \\
\hline YNDLKEETFK & $38-47$ \\
\hline YNDLKEETFKAVAMITFAQYLQR & $38-60$ \\
\hline AVAMITFAQYLQR & $48-60$ \\
\hline CSYEGLSK & $61-68$ \\
\hline CVANEDAPECSKPLPSIILDEICQVEK & $80-106$ \\
\hline ADPERNECFLSFK & $121-133$ \\
\hline VSFLGHFIYSVAR & $160-172$ \\
\hline RHPFLYAPAILSFAVDFEHALQSCCK & $173-198$ \\
\hline HPFLYAPAILSFAVDFEHALQSCCK & $174-198$ \\
\hline SQCIMEAEFDEKPADLPSLVEK & $315-336$ \\
\hline SFEAGHDAFMAEFVYEYSR & $346-364$ \\
\hline RHPEFSIQLIMR & $365-376$ \\
\hline
\end{tabular}




\begin{tabular}{|c|c|}
\hline HPEFSIQLIMR & $366-376$ \\
\hline TDNPAECYANAQEQLNQHIK & $391-410$ \\
\hline KMPQVPTDLLLETGK & $442-456$ \\
\hline MPQVPTDLLLETGK & $443-456$ \\
\hline MPQVPTDLLLETGKK & $443-457$ \\
\hline MACSEGYLSIVIHDTCR & $474-490$ \\
\hline YVPPPFNPDMFSFDEK & $525-540$ \\
\hline LCSAPAEER & $541-549$ \\
\hline RKPQMTEEQIK & $563-573$ \\
\hline TIADGFTAMVDK & $574-585$ \\
\hline QSDINTCFGEEGANLIVQSR & $589-608$ \\
\hline YNDLKEETFK & $38-47$ \\
\hline YNDLKEETFKAVAMITFAQYLQR & $38-60$ \\
\hline AVAMITFAQYLQR & $48-60$ \\
\hline CVANEDAPECSKPLPSIILDEICQVEK & $80-106$ \\
\hline VSFLGHFIYSVAR & $160-172$ \\
\hline ESDVGACLDTK & $199-209$ \\
\hline APFSEVSK & $254-261$ \\
\hline FVHDSIGVHK & $262-271$ \\
\hline SFEAGHDAFMAEFVYEYSR & 346-364 \\
\hline RHPEFSIQLIMR & $365-376$ \\
\hline TDNPAECYANAQEQLNQHIK & $391-410$ \\
\hline KMPQVPTDLLLETGK & $442-456$ \\
\hline MPQVPTDLLLETGK & $443-456$ \\
\hline MPQVPTDLLLETGKK & $443-457$ \\
\hline MACSEGYLSIVIHDTCR & $474-490$ \\
\hline YVPPPFNPDMFSFDEK & $525-540$ \\
\hline LCSAPAEER & $541-549$ \\
\hline LLINLIK & $556-562$ \\
\hline KPQMTEEQIK & $564-573$ \\
\hline TIADGFTAMVDK & $574-585$ \\
\hline QSDINTCFGEEGANLIVQSR & $589-608$ \\
\hline DDNKVEDIWSFLSK & $275-288$ \\
\hline VITPSFVDIFISK & $219-231$ \\
\hline YNDLKEETFKAVAMITFAQYLQR & $38-60$ \\
\hline CSYEGLSKLVKDVVDLAQK & $61-79$ \\
\hline LVKDVVDLAQK & $69-79$ \\
\hline CVANEDAPECSKPLPSIILDEICQVEK & $80-106$ \\
\hline CVANEDAPECSKPLPSIILDEICQVEKLR & $80-108$ \\
\hline VSFLGHFIYSVAR & $160-172$ \\
\hline RHPFLYAPAILSFAVDFEHALQSCCK & $173-198$ \\
\hline QQYFCGILKQFGDRVFQAR & $224-242$ \\
\hline QLIYLSQKYPK & $243-253$ \\
\hline APFSEVSK & $254-261$ \\
\hline IKDCCEKPIVER & $303-314$ \\
\hline TDNPAECYANAQEQLNQHIK & $391-410$ \\
\hline KMoxPQVPTDLLLETGK & $442-456$ \\
\hline MPQVPTDLLLETGKK & $443-457$ \\
\hline LLINLIKR & $556-563$ \\
\hline QSDINTCFGEEGANLIVQSR & $589-608$ \\
\hline
\end{tabular}




\begin{tabular}{|c|c|}
\hline YNDLKEETFK & $38-47$ \\
\hline AVAMITFAQYLQR & $48-60$ \\
\hline VSFLGHFIYSVAR & $160-172$ \\
\hline TDNPAECYANAQEQLNQHIK & $391-410$ \\
\hline MoxPQVPTDLLLETGK & $443-456$ \\
\hline YVPPPFNPDMFSFDEK & $525-540$ \\
\hline LCSAPAEER & $541-549$ \\
\hline LLINLIK & $556-562$ \\
\hline TIADGFTAMVDK & $574-585$ \\
\hline SAGWNIPIGTLLHR & $141-154$ \\
\hline GAIEWEGIESGSVEQAVAK & $155-173$ \\
\hline VEDIWSFLSK & $279-288$ \\
\hline AQSDFGVDTK & $289-298$ \\
\hline SDFHLFGPPGK & 299-309 \\
\hline DQLTPSPR & $353-360$ \\
\hline TDERPASYFAVAVAR & $443-457$ \\
\hline VITPSFVDIFISK & $219-231$ \\
\hline KGTEFTVNDLQGK & $119-131$ \\
\hline SAGWNIPIGTLLHR & 141-154 \\
\hline GAIEWEGIESGSVEQAVAK & $155-173$ \\
\hline FFSASCVPGATIEQK & $174-188$ \\
\hline NAPYSGYSGAFHCLK & $204-218$ \\
\hline VEDIWSFLSK & $279-288$ \\
\hline AQSDFGVDTK & $289-298$ \\
\hline SDFHLFGPPGK & 299-309 \\
\hline TDERPASYFAVAVAR & $443-457$ \\
\hline LCQLCQGSGGIPPEK & $517-531$ \\
\hline NLQMoxDDFELLCTDGR & $579-593$ \\
\hline ECNLAEVPTHAVVVRPEK & $602-619$ \\
\hline FYTVISSLK & $680-688$ \\
\hline EDFEGPFR & $122-129$ \\
\hline VITPSFVDIFISK & $219-231$ \\
\hline ASNARPPSVYVFPPPTEQLNGNQR & $316-339$ \\
\hline LLINLIK & $556-562$ \\
\hline KGTEFTVNDLQGK & $119-131$ \\
\hline GTEFTVNDLQGK & $120-131$ \\
\hline SAGWNIPIGTLLHR & $141-154$ \\
\hline GAIEWEGIESGSVEQAVAK & $155-173$ \\
\hline FFSASCVPGATIEQK & $174-188$ \\
\hline DDNKVEDIWSFLSK & $275-288$ \\
\hline VEDIWSFLSK & $279-288$ \\
\hline AQSDFGVDTK & $289-298$ \\
\hline SDFHLFGPPGK & 299-309 \\
\hline TDERPASYFAVAVAR & $443-457$ \\
\hline LCQLCQGSGGIPPEK & $517-531$ \\
\hline ECNLAEVPTHAVVVRPEK & $602-619$ \\
\hline FYTVISSLK & $680-688$ \\
\hline VITPSFVDIFISK & $219-231$ \\
\hline LLINLIK & $556-562$ \\
\hline
\end{tabular}




\begin{tabular}{|c|c|}
\hline KCNNLRDLTQQER & $38-50$ \\
\hline GTEFTVNDLQGK & $120-131$ \\
\hline SAGWNIPIGTLLHR & 141-154 \\
\hline GAIEWEGIESGSVEQAVAK & $155-173$ \\
\hline NAPYSGYSGAFHCLK & 204-218 \\
\hline AQSDFGVDTK & 289-298 \\
\hline SDFHLFGPPGKK & 299-310 \\
\hline DQLTPSPR & $353-360$ \\
\hline TDERPASYFAVAVAR & $443-457$ \\
\hline IRDLLER & $623-629$ \\
\hline AGKEPGLQIWR & $58-68$ \\
\hline EPGLQIWR & $61-68$ \\
\hline IEKFDLVPVPK & $69-79$ \\
\hline HVVPNEVTVQR & 174-184 \\
\hline HTQVQVLPESGETPLFK & $370-386$ \\
\hline IEKVPFDAATLHTSR & $413-427$ \\
\hline TPSAAYLWVGR & $594-604$ \\
\hline GSNSAELSGAQELLK & $605-619$ \\
\hline SAGWNIPIGTLLHR & 141-154 \\
\hline VEDIWSFLSK & $279-288$ \\
\hline SDFHLFGPPGK & 299-309 \\
\hline DQLTPSPR & $353-360$ \\
\hline ECNLAEVPTHAVVVRPEK & $602-619$ \\
\hline FFSASCVPGATIEQK & $174-188$ \\
\hline TDERPASYFAVAVAR & $443-457$ \\
\hline CGPGLVLLGSAVR & $164-176$ \\
\hline KGTEFTVNDLQGK & $119-131$ \\
\hline GTEFTVNDLQGK & $120-131$ \\
\hline SAGWNIPIGTLLHR & 141-154 \\
\hline GAIEWEGIESGSVEQAVAK & $155-173$ \\
\hline FFSASCVPGATIEQK & $174-188$ \\
\hline NAPYSGYSGAFHCLK & $204-218$ \\
\hline DGKGDVAFVK & $219-228$ \\
\hline VEDIWSFLSK & $279-288$ \\
\hline SDFHLFGPPGK & 299-309 \\
\hline SDFHLFGPPGKK & 299-310 \\
\hline TDERPASYFAVAVAR & $443-457$ \\
\hline NLQMoxDDFELLCTDGR & $579-593$ \\
\hline ANVMoxDYR & 595-601 \\
\hline ECNLAEVPTHAVVVRPEK & $602-619$ \\
\hline FYTVISSLK & $680-688$ \\
\hline TCNPSDILQMoxCSFLEGK & $689-705$ \\
\hline KGTEFTVNDLQGKTSCHTGLGR & $119-140$ \\
\hline GTEFTVNDLQGK & $120-131$ \\
\hline SAGWNIPIGTLLHR & 141-154 \\
\hline GAIEWEGIESGSVEQAVAK & $155-173$ \\
\hline FFSASCVPGATIEQKLCR & 174-191 \\
\hline TDERPASYFAVAVAR & $443-457$ \\
\hline LCQLCQGSGGIPPEK & $517-531$ \\
\hline SAGWNIPIGTLLHR & 141-154 \\
\hline
\end{tabular}




\begin{tabular}{|c|c|}
\hline GAIEWEGIESGSVEQAVAK & $155-173$ \\
\hline DDNKVEDIWSFLSK & $275-288$ \\
\hline VEDIWSFLSK & $279-288$ \\
\hline TDERPASYFAVAVAR & $443-457$ \\
\hline ECNLAEVPTHAVVVRPEK & $602-619$ \\
\hline EFLGDKFYTVISSLK & $674-688$ \\
\hline TNCDLLHDHGEADFLK & $418-433$ \\
\hline APFSEVSK & $254-261$ \\
\hline IAKGYESLLEK & 377-387 \\
\hline AVAMITFAQYLQR & $48-60$ \\
\hline VSFLGHFIYSVAR & $160-172$ \\
\hline APFSEVSK & $254-261$ \\
\hline SQCIMoxEAEFDEKPADLPSLVEK & $315-336$ \\
\hline TDNPAECYANAQEQLNQHIK & $391-410$ \\
\hline MPQVPTDLLLETGK & $443-456$ \\
\hline YVPPPFNPDMFSFDEK & $525-540$ \\
\hline TIADGFTAMVDK & $574-585$ \\
\hline AVAMITFAQYLQR & $48-60$ \\
\hline VSFLGHFIYSVAR & $160-172$ \\
\hline ESDVGACLDTK & 199-209 \\
\hline QLIYLSQKYPK & $243-253$ \\
\hline APFSEVSK & $254-261$ \\
\hline SFEAGHDAFMAEFVYEYSR & 346-364 \\
\hline KMoxPQVPTDLLLETGKK & $442-457$ \\
\hline LCSAPAEER & 541-549 \\
\hline TPSAAYLWVGR & $594-604$ \\
\hline APFSEVSK & $254-261$ \\
\hline ILSPVCGTDGFTYDNECGICAHNAEQR & $179-205$ \\
\hline ILLPVCGTDGFTYDNECGICAHNAQHGTEVKK & $245-276$ \\
\hline ELFDPVIEDR & $87-96$ \\
\hline LSVEALGSLGGDLK & $157-170$ \\
\hline FCTGLTQIETLFK & $253-265$ \\
\hline FGEVLKR & $308-314$ \\
\hline LLIEMoxEK & $359-365$ \\
\hline GQSIDDLMoxPAQK & $370-381$ \\
\hline EFVEDFIWPAVR & $68-79$ \\
\hline GNPTVEVDLYTNKGLFR & $16-32$ \\
\hline AAVPSGASTGIYEALELR & $33-50$ \\
\hline AAVPSGASTGIYEALELRDNDKTR & $33-56$ \\
\hline HIADLAGNPEVILPVPAFNVINGGSHAGNK & 133-162 \\
\hline IGAEVYHNLK & $184-193$ \\
\hline DATNVGDEGGFAPNILENKEALELLK & $203-228$ \\
\hline EALELLK & $222-228$ \\
\hline VVIGMoxDVAASEFYR & $240-253$ \\
\hline DGKYDLDFKSPDDPSR & $254-269$ \\
\hline LAQSNGWGVMoxVSHR & $359-372$ \\
\hline IEEELGSK & $413-420$ \\
\hline VNQIGSVTESIQACK & $344-358$ \\
\hline
\end{tabular}


VLDAVRGSPAANVAVK

39-54

GSPAANVAVKVFK

GSPAANVAVKVFKK

AADGTWQDFATGK

VEFDTSSYWK
45-57

45-58

59-71

94-103

FVEGLPINDFSR

299-310

ITVVGVGQVGMACAISILGK

23-42

LITPVAAGSTVPSNK

$8-22$

ITVVGVGQVGMOXACAISILGK

23-42

GLCDELALVDVLEDK

43-57

GEMMDLQHGSLFLQTHK

60-76

IVADKDYAVTANSK

77-90

IVVVTAGVR

91-99

YSPNCTILVVSNPVDILTYVTWK

127-149

VIGSGCNLDTAR

158-169

QVVESAYEVIR

233-243

VHSVSTLVK

270-278

GTYGIENDVFLSLPCVLSASGLTSVINQK

279-307

SADTLWSIQK

319-328

SADGCWSNYGK

151-161

VGGGQTISVMK

162-172

ATIVPVPDGSVHIGQR

252-262

268-277

LGLSNLDVAK

287-299

CSTIIDAAFGSLK

4-11

DMVDVYLETVK

36-46

51-63

DAIAQFESSAVGK

69-82

LADNLDTLSAAAAK

120-130

IRPFLDQFSAK

131-139

WTEELEQYR

142-150

LTPVAQELK

164-172

LTPVAEEAR

185-194

208-218

GIPQASEYQAK

219-227

230-238

MTPLVQEFR

252-261

LISFLDELQK

4-15

4-23

VAPTITLFPPSKEELNEATK

51-63

69-82

LADNLDTLSAAAAK

72-84

GAFTGEISPAMIK

100-112

HVFGESDELIGQK

111-120 
ELNEDGTCADSYSFVFSR
QIDLCLDR
LADNLDTLSAAAAK
MTPLVQEFR
AADGTWQDFATGK
TTEFGEIHELTTEEQFVEGVYR
VEFDTSSYWK
LADSHNFDEYMK
ALGVGFAMR
ALGVGFAMOXR
QVGNVTKPTVIISSEGDKVVIR
TQSTFKNTEISFK
NTEISFK
LGEEFDETTPDDR
SVVTLDGDKLVHVQK
MOXVMOXTLTFGDVVAVR

ALQFAMOXAEYNR

RQLVSGIK

YILQVEIGR

SIPPACDK

DYSPVCGTDGK

TYPNECVLCLSNSEENKNVQIYK

DYSPVCGTDGK

TYPNECVLCLSNSEENKNVQIYK

APSVDIGTCR

QFDPSDFQDETVTR

ASPETAEIFFQK

AFLFPDTSR

ASPETAEIFFQK

ECQSVCIFEGR

HNGQIWVLENDR
143-160

177-184

69-82

230-238

59-71

72-93

94-103

11-22

23-31

23-31

32-53

54-66

60-66

67-79

83-97

114-127

47-57

75-82

83-91

1-8

18-28

29-51

18-28

29-51

45-54

604-617

69-80

57-65

69-80

329-339

648-659

51-59

80-106

160-172

262-271

303-314

365-376

375-389 


\begin{tabular}{|c|c|}
\hline CVANEDAPECSKPLPSIILDEICQVEK & $80-106$ \\
\hline ESDVGACLDTK & 199-209 \\
\hline LLINLIK & $556-562$ \\
\hline AFLFPDTSR & $57-65$ \\
\hline DIPVNPICIYR & $32-42$ \\
\hline YNDLKEETFK & $38-47$ \\
\hline YNDLKEETFKAVAMITFAQYLQR & $38-60$ \\
\hline AVAMITFAQYLQR & $48-60$ \\
\hline LVKDVVDLAQK & $69-79$ \\
\hline CVANEDAPECSKPLPSIILDEICQVEK & $80-106$ \\
\hline VSFLGHFIYSVAR & $160-172$ \\
\hline HPFLYAPAILSFAVDFEHALOSCCK & $174-198$ \\
\hline QQYFCGILKQFGDRVFQAR & $224-242$ \\
\hline APFSEVSK & $254-261$ \\
\hline FVHDSIGVHK & $262-271$ \\
\hline MMSNLCSQQDVFSGK & 288-302 \\
\hline IKDCCEKPIVER & 303-314 \\
\hline SQCIMEAEFDEKPADLPSLVEK & $315-336$ \\
\hline SFEAGHDAFMAEFVYEYSR & 346-364 \\
\hline RHPEFSIQLIMR & $365-376$ \\
\hline HPEFSIQLIMR & $366-376$ \\
\hline KMPQVPTDLLLETGK & $442-456$ \\
\hline KMPQVPTDLLLETGKK & $442-457$ \\
\hline MPQVPTDLLLETGK & $443-456$ \\
\hline MPQVPTDLLLETGKK & $443-457$ \\
\hline MACSEGYLSIVIHDTCR & $474-490$ \\
\hline RPCFTAMGVDTK & $513-524$ \\
\hline YVPPPFNPDMFSFDEK & $525-540$ \\
\hline TIADGFTAMVDK & $574-585$ \\
\hline QSDINTCFGEEGANLIVQSR & $589-608$ \\
\hline MoxREIVHIQAGQCGNQIGAK & $1-19$ \\
\hline EIVHIQAGQCGNQIGAK & $3-19$ \\
\hline SGPFGQIFRPDNFVFGQSGAGNNWAK & $78-103$ \\
\hline GHYTEGAELVDSVLDVVR & $104-121$ \\
\hline GHYTEGAELVDSVLDVVRK & $104-122$ \\
\hline LTTPTYGDLNHLVSATMSGVTTCLR & 217-241 \\
\hline FPGQLNADLR & $242-251$ \\
\hline FPGQLNADLRK & $242-252$ \\
\hline KLAVNMoxVPFPR & $252-262$ \\
\hline LAVNMoxVPFPR & 253-262 \\
\hline LHFFMoxPGFAPLTSR & $263-276$ \\
\hline EVDEQMoxLNVQNK & $325-336$ \\
\hline NSSYFVEWIPNNVK & $337-350$ \\
\hline TAVCDIPPR & $351-359$ \\
\hline MSATFIGNSTAIQELFK & $363-379$ \\
\hline MoxSATFIGNSTAIQELFKR & $363-380$ \\
\hline ISEQFTAMoxFR & $381-390$ \\
\hline AVLVDLEPGTMoxDSVR & $63-77$ \\
\hline GHYTEGAELVDSVLDVVR & $104-121$ \\
\hline GHYTEGAELVDSVLDVVRK & $104-122$ \\
\hline
\end{tabular}




$\begin{array}{lc}\text { FPGQLNADLR } & 242-251 \\ \text { FPGQLNADLRK } & 242-252 \\ \text { KLAVNMOXVPFPR } & 252-262 \\ \text { LAVNMOXVPFPR } & 253-262 \\ \text { ALTVPELTQQMOXFDAK } & 283-297 \\ \text { NSSYFVEWIPNNVK } & 337-350 \\ \text { MASTFIGNSTAIQELFK } & 363-379 \\ \text { MOXASTFIGNSTAIQELFKR } & 363-380 \\ \text { QLFHPEQLITGK } & 85-96 \\ \text { NLDIERPTYTNLNR } & 216-229 \\ \text { FDGALNVDLTEFQTNLVPYPR } & 244-264 \\ \text { QLFHPEQLITGK } & 85-96 \\ \text { NLDIERPTYTNLNR } & 216-229 \\ \text { FDGALNVDLTEFQTNLVPYPR } & 244-264 \\ \text { DVNAAIATIK } & 327-336 \\ \text { AFVHWYVGEGMEEGEFSEAR } & 403-422 \\ \text { GHYTEGAELVDSVLDVVR } & 104-121 \\ \text { FPGQLNADLR } & 242-251 \\ \text { LAVNMOXVPFPR } & 253-262\end{array}$

AGGVASGFR

$165-173$

HVVPNEVTVQR 174-184

VFVSEEGAER 254-263

HTQVQVLPESGETPLFK 370-386

VPFDAATLHTSR 416-427

AVELDPAASQLNSNDAFVLK 574-593

EPDNFWVALGGK 633-644

YIETDPASR 726-734

FFSASCVPGATIEQK 174-188

AQSDFGVDTK 289-298

DQLTPSPR 353-360

LLINLIK 556-562

AGKEPGLQIWR $58-68$

EPGLQIWR $\quad 61-68$

IEKFDLVPVPK $\quad 69-79$

NLYGDFFTGDSYLVLNTIR $\quad 80-98$

GAAAIFTVQMOXDDYLQGK 122-138

AGGVASGFR 165-173

HTQVQVLPESGETPLFK 370-386

DKDQTEGLGEAYISGHVAK 394-412

IEKVPFDAATLHTSR $\quad$ 413-427

VPFDAATLHTSR 416-427

AVELDPAASQLNSNDAFVLK 574-593

TPSAAYLWVGR 594-604

GSNSAELSGAQELLK 605-619 


\begin{tabular}{|c|c|}
\hline EPDNFWVALGGK & $633-644$ \\
\hline DAQEEEKTEALK & $710-721$ \\
\hline YIETDPASR & 726-734 \\
\hline YNDLKEETFK & $38-47$ \\
\hline AVAMITFAQYLQR & $48-60$ \\
\hline LLINLIK & $556-562$ \\
\hline VITPSFVDIFISK & $219-231$ \\
\hline YLLEDTVR & $154-161$ \\
\hline APLSFDTPSDVAASFMASLSQSVER & $193-217$ \\
\hline SDFHLFGPPGK & 299-309 \\
\hline DQLTPSPR & $353-360$ \\
\hline KGTEFTVNDLQGK & $119-131$ \\
\hline GTEFTVNDLQGK & $120-131$ \\
\hline SAGWNIPIGTLLHR & 141-154 \\
\hline GAIEWEGIESGSVEQAVAK & $155-173$ \\
\hline FFSASCVPGATIEQK & $174-188$ \\
\hline NAPYSGYSGAFHCLK & $204-218$ \\
\hline DGKGDVAFVK & $219-228$ \\
\hline HTTVNENAPDQK & $229-240$ \\
\hline DDNKVEDIWSFLSK & $275-288$ \\
\hline VEDIWSFLSK & $279-288$ \\
\hline AQSDFGVDTK & $289-298$ \\
\hline SDFHLFGPPGK & 299-309 \\
\hline SDFHLFGPPGKK & $299-310$ \\
\hline SDFHLFGPPGKKDPVLK & 299-315 \\
\hline DPVLKDLLFK & $311-320$ \\
\hline DPVLKDLLFKDSAIMLK & $311-327$ \\
\hline VPSLMDSQLYLGFEYYSAIQSMoxR & $329-351$ \\
\hline KDQLTPSPR & $352-360$ \\
\hline TDERPASYFAVAVAR & $443-457$ \\
\hline GDVAFIQHSTVEENTGGK & $554-571$ \\
\hline NLQMoxDDFELLCTDGR & $579-593$ \\
\hline DLLFKDLTK & $653-661$ \\
\hline EFLGDKFYTVISSLK & $674-688$ \\
\hline FYTVISSLK & $680-688$ \\
\hline TCNPSDILQMCSFLEGK & $689-705$ \\
\hline AVAMITFAQYLQR & $48-60$ \\
\hline LLINLIK & $556-562$ \\
\hline VITPSFVDIFISK & $219-231$ \\
\hline NAPYSGYSGAFHCLK & $204-218$ \\
\hline IQWCAVGKDEK & $364-374$ \\
\hline IRDLLER & $623-629$ \\
\hline KGTEFTVNDLQGK & $119-131$ \\
\hline GTEFTVNDLQGK & $120-131$ \\
\hline SAGWNIPIGTLLHR & $141-154$ \\
\hline GAIEWEGIESGSVEQAVAK & $155-173$ \\
\hline
\end{tabular}




\begin{tabular}{|c|c|}
\hline FFSASCVPGATIEQK & $174-188$ \\
\hline NAPYSGYSGAFHCLK & $204-218$ \\
\hline DGKGDVAFVK & $219-228$ \\
\hline DDNKVEDIWSFLSK & $275-288$ \\
\hline VEDIWSFLSK & $279-288$ \\
\hline AQSDFGVDTK & $289-298$ \\
\hline SDFHLFGPPGK & 299-309 \\
\hline DPVLKDLLFK & $311-320$ \\
\hline TDERPASYFAVAVAR & $443-457$ \\
\hline KDSNVNWNNLK & $458-468$ \\
\hline TGTCNFDEYFSEGCAPGSPPNSR & $494-516$ \\
\hline GDVAFIQHSTVEENTGGK & $554-571$ \\
\hline NLQMoxDDFELLCTDGR & $579-593$ \\
\hline ECNLAEVPTHAVVVRPEK & $602-619$ \\
\hline EFLGDKFYTVISSLK & $674-688$ \\
\hline FYTVISSLK & $680-688$ \\
\hline TCNPSDILQMCSFLEGK & $689-705$ \\
\hline LKPIAAEVYEHTEGSTTSYYAVAVVK & $93-118$ \\
\hline GTEFTVNDLQGK & $120-131$ \\
\hline TDERPASYFAVAVAR & $443-457$ \\
\hline APSVDIGTCR & $45-54$ \\
\hline LKPIAAEVYEHTEGSTTSYYAVAVVK & $93-118$ \\
\hline GTEFTVNDLQGK & $120-131$ \\
\hline SAGWNIPIGTLLHR & $141-154$ \\
\hline GAIEWEGIESGSVEQAVAK & $155-173$ \\
\hline VEDIWSFLSK & $279-288$ \\
\hline KDQLTPSPR & $352-360$ \\
\hline TDERPASYFAVAVAR & $443-457$ \\
\hline ECNLAEVPTHAVVVRPEK & $602-619$ \\
\hline LKPIAAEVYEHTEGSTTSYYAVAVVK & $93-118$ \\
\hline GTEFTVNDLQGK & $120-131$ \\
\hline SAGWNIPIGTLLHR & $141-154$ \\
\hline GAIEWEGIESGSVEQAVAK & $155-173$ \\
\hline FFSASCVPGATIEQK & $174-188$ \\
\hline NAPYSGYSGAFHCLK & $204-218$ \\
\hline VEDIWSFLSK & $279-288$ \\
\hline AQSDFGVDTK & $289-298$ \\
\hline SDFHLFGPPGK & 299-309 \\
\hline TDERPASYFAVAVAR & $443-457$ \\
\hline FYTVISSLK & $680-688$ \\
\hline TCNPSDILQMCSFLEGK & $689-705$ \\
\hline YNDLKEETFK & $38-47$ \\
\hline AVAMoxITFAQYLQR & $48-60$ \\
\hline LVKDVVDLAQK & $69-79$ \\
\hline CVANEDAPECSKPLPSIILDEICQVEK & $82-106$ \\
\hline VSFLGHFIYSVAR & $160-172$ \\
\hline APFSEVSK & $254-261$ \\
\hline MMoxSNLCSQQDVFSGK & $288-302$ \\
\hline RHPEFSIQLIMoxR & $365-376$ \\
\hline KMoxPQVPTDLLLETGK & $442-456$ \\
\hline
\end{tabular}




\begin{tabular}{|c|c|}
\hline MoxPQVPTDLLLETGK & $443-456$ \\
\hline RPCFTAMGVDTK & $513-524$ \\
\hline YVPPPFNPDMoxFSFDEK & $525-540$ \\
\hline LLINLIK & $556-562$ \\
\hline TIADGFTAMVDK & $574-585$ \\
\hline QSDINTCFGEEGANLIVQSR & $589-608$ \\
\hline LVKDVVDLAQK & $69-79$ \\
\hline VSFLGHFIYSVAR & $160-172$ \\
\hline APFSEVSK & $254-261$ \\
\hline RHPEFSIQLIMR & $365-376$ \\
\hline AVAMITFAQYLQR & $48-60$ \\
\hline LVKDVVDLAQK & $69-79$ \\
\hline CVANEDAPECSKPLPSIILDEICQVEK & $80-106$ \\
\hline VSFLGHFIYSVAR & $160-172$ \\
\hline APFSEVSK & $254-261$ \\
\hline RHPEFSIQLIMoxR & $365-376$ \\
\hline KMoxPQVPTDLLLETGK & $442-456$ \\
\hline KMoxPQVPTDLLLETGKK & $442-457$ \\
\hline YVPPPFNPDMFSFDEK & $525-540$ \\
\hline LLINLIK & $556-562$ \\
\hline TIADGFTAMVDK & $574-585$ \\
\hline AVAMITFAQYLQR & $48-60$ \\
\hline LVKDVVDLAQK & $69-79$ \\
\hline CVANEDAPECSKPLPSIILDEICQVEK & $80-106$ \\
\hline VSFLGHFIYSVAR & $160-172$ \\
\hline HPFLYAPAILSFAVDFEHALQSCCK & 174-198 \\
\hline MoxPQVPTDLLLETGK & $443-456$ \\
\hline YVPPPFNPDMoxFSFDEK & $525-540$ \\
\hline SAGWNIPIGTLLHR & 141-154 \\
\hline GAIEWEGIESGSVEQAVAK & $155-173$ \\
\hline NAPYSGYSGAFHCLK & $204-218$ \\
\hline FYTVISSLK & $680-688$ \\
\hline TCNPSDILQMCSFLEGK & $689-705$ \\
\hline APFSEVSK & $254-261$ \\
\hline YVPPPFNPDMFSFDEK & $525-540$ \\
\hline AVAMITFAQYLQR & $48-60$ \\
\hline ESDVGACLDTK & 199-209 \\
\hline YVPPPFNPDMFSFDEK & $525-540$ \\
\hline YNDLKEETFK & $38-47$ \\
\hline LVKDVVDLAQK & $69-79$ \\
\hline ESDVGACLDTK & $199-209$ \\
\hline RPCFTAMGVDTK & $513-524$ \\
\hline YVPPPFNPDMFSFDEK & $525-540$ \\
\hline TIADGFTAMVDK & $574-585$ \\
\hline SAGWNIPIGTLLHR & 141-154 \\
\hline AQSDFGVDTK & $289-298$ \\
\hline NTGIICTIGPASR & $43-55$ \\
\hline IYVDDGLISLLVK & $173-185$ \\
\hline
\end{tabular}


HVMIDCSPYLQVVR $\quad$ 89-102

DGNTMVACPR 103-112

ILSPVCGTDGFTYDNECGICAHNAEQR 179-205

CREEVPELDCSK 347-358

EEVPELDCSK 349-358

VSPICTMOXEYVPHCGSDGVTYSNR $\quad 428-450$

CFFCNAYVQSNR $\quad 451-462$

DGTSWVACPR 38-47

QEIPEIDCDQYPTR 218-231

CFFCNAYVQSNR $\quad 451-462$

HVMIDCSPYLQVVR $\quad$ 89-102

DGNTMVACPR 103-112

QEIPEIDCDQYPTR 218-231

VSPICTMEYVPHCGSDGVTYSNR $\quad 428-450$

CFFCNAYVQSNR $\quad 451-462$

DGTSWVACPR $\quad 38-47$

HVMOXIDCSPYLQVVR $\quad$ 89-102

SPCVASCNIPVVSGR 199-213

HVMIDCSPYLQVVR $\quad$ 89-102

DNLDNNIPSSLR $159-170$

DGNTMVACPR 103-112

HYTIAALLSPFSYSTTAVVSDPQE $127-150$

DNLDNNIPSSLR $159-170$

EDGGGWWYNR 400-409

AVAMITFAQYLQR $\quad 48-60$

HPLDELGVLCPTGCELQTTLLK 71-92

TVKPVLR 96-102

DNLDNNIPSSLR $159-170$

AWDEYKR 269-275

VSALYGGFTIHNEGNK 325-340

YQLSVSNYK 341-349

DNDGWLTTDPR 384-394

EDGGGWWYNR 400-409

YYWGGTYSWDMAK 419-431

AVAMITFAQYLQR 48-60

LLPDLESFFTHDSVSTSSR 367-385

LVKDVVDLAQK $\quad 69-79$

FVHDSIGVHK 262-271

SFEAGHDAFMOXAEFVYEYSR 346-364

RHPEFSIQLIMR 365-376

KMOXPQVPTDLLLETGK 442-456

MPQVPTDLLLETGK 443-456

RPCFTAMOXGVDTK 513-524

YVPPPFNPDMFSFDEK $\quad 525-540$

FCTGLTQIETLFK 253-265

LSVEALGSLGGDLK 157-170 
FCTGLTQIETLFK

GQSIDDLMOXPAQK

253-265

370-381

ELFDPVIEDR

LSVEALGSLGGDLK

FCTGLTQIETLFK

FGEVLKR

GTGGVDTAAVGGVFDVSNADR

GNPTVEVDLYTNK

AAVPSGASTGIYEALELR

NVNVVEQEKIDK

LMLEMDGTENK

FGANAILGVSLAVCK

HIADLAGNPEVILPVPAFNVINGGSHAGNK

IGAEVYHNLK

DATNVGDEGGFAPNILENK

EALELLK

VVIGMDVAASEFYR

DGKYDLDFK

DGKYDLDFKSPDDPSR

NYPVVSIEDPFDQDDWAAWK

KFTASVGIQVVGDDLTVTNPK

SCNCLLLK

VNQIGSVTESLQACK

LAQSNGWGVMVSHR

SGETEDTFIADLVVGLCTGQIK

IEEELGSK

AAVPSGASTGIYEALELR

FGANAILGVSLAVCK

IGAEVYHNLK

DGKYDLDFK

DGKYDLDFKSPDDPSR

LAQENGWGVMoxVSHR

FGANAILGVSLAVCK

VNQIGSVTESIQACK

SGETEDTFIADLVVGLCTGQIK

LGDVYVNDAFGTAHR

ITLPVDFITADK

EVIPTDKEEVAFK

SQGAALDKYAK

VVVVGNPANTNCLIASK

SAPSIPKENFSCLTR

LGVTSNDVK

EVGVYEAIKDDSWLKGDFILTVQQR

GDFILTVQQR

FVEGLPINDFSR
87-96

157-170

253-265

308-314

321-341

16-28

33-50

81-92

93-103

106-120

133-162

184-193

203-221

222-228

240-253

254-262

254-269

286-305

306-326

336-343

344-358

359-372

373-394

413-420

33-50

106-120

184-193

254-262

254-269

359-372

106-120

344-358

373-394

157-171

280-291

67-79

111-121

126-142

143-157

171-179

206-230

221-230

299-310 


\begin{tabular}{|c|c|}
\hline LITPVAAGSTVPSNK & $8-22$ \\
\hline GLCDELALVDVLEDK & $43-57$ \\
\hline GEMoxMoxDLQHGSLFLQTHK & $60-76$ \\
\hline IVADKDYAVTANSK & $77-90$ \\
\hline IVVVTAGVR & 91-99 \\
\hline VIGSGCNLDTAR & $158-169$ \\
\hline QVVESAYEVIR & $233-243$ \\
\hline VHSVSTLVK & $270-278$ \\
\hline LKDDEVAQLK & $308-317$ \\
\hline LKDDEVAQLKK & $308-318$ \\
\hline SADTLWSIQK & $319-328$ \\
\hline GLCDELALVDVLEDK & $43-57$ \\
\hline VIGSGCNLDTAR & $158-169$ \\
\hline QVVESAYEVIR & $233-243$ \\
\hline DAGYEFDICFTSVQK & $47-61$ \\
\hline HYGALTGLNK & $91-100$ \\
\hline YADLTEDQLPTCESLKDTIAR & $142-162$ \\
\hline VLIAAHGNSLR & 181-191 \\
\hline HLEGMSEEAIMoxELNLPTGIPIVYELDK & $196-222$ \\
\hline SLGELIHTLNGAK & $20-32$ \\
\hline IGVAAQNCYK & $59-68$ \\
\hline GAFTGEISPAMoxIK & $72-84$ \\
\hline DIGAAWVILGHSER & $85-98$ \\
\hline HVFGESDELIGQK & $100-112$ \\
\hline VVLAYEPVWAIGTGK & $160-174$ \\
\hline TATPQQAQEVHEK & $175-187$ \\
\hline SHVSDAVAQSTR & $194-205$ \\
\hline ELASQHDVDGFLVGGASLKPEFVDIINAK & $219-247$ \\
\hline LKPIAAEVYEHTEGSTTSYYAVAVVK & $93-118$ \\
\hline GTEFTVNDLQGK & $120-131$ \\
\hline FFSASCVPGATIEQK & 174-188 \\
\hline QSHGSCSFVACR & $33-44$ \\
\hline MoxAAAIGLRVVGR & $1-12$ \\
\hline KAADGTWQDFATGK & $58-71$ \\
\hline AADGTWQDFATGK & $59-71$ \\
\hline TTEFGEIHELTTEEQFVEGVYR & $72-93$ \\
\hline VEFDTSSYWK & $94-103$ \\
\hline GLGLSPFHEYADVVFTANDSGHR & $104-126$ \\
\hline DAIAQFESSAVGK & $51-63$ \\
\hline LADNLDTLSAAAAK & $69-82$ \\
\hline LTPVAQELK & $142-150$ \\
\hline LTPVAEEAR & $164-172$ \\
\hline NLAPYSDELR & 185-194 \\
\hline VMoxEQLSNLR & $219-227$ \\
\hline MoxTPLVQEFR & $230-238$ \\
\hline LTPYAENLK & $241-249$ \\
\hline
\end{tabular}




\begin{tabular}{|c|c|}
\hline LISFLDELQK & $252-261$ \\
\hline AADGTWQDFATGK & $59-71$ \\
\hline TTEFGEIHELTTEEQFVEGVYR & $72-93$ \\
\hline VEFDTSSYWK & $94-103$ \\
\hline VAPTITLFPPSK & $4-15$ \\
\hline IRDMVDVYLETVK & $34-46$ \\
\hline DMVDVYLETVK & $36-46$ \\
\hline DAIAQFESSAVGK & $51-63$ \\
\hline LADNLDTLSAAAAK & $69-82$ \\
\hline LREDMAPYYK & $83-92$ \\
\hline IRPFLDQFSAK & $120-130$ \\
\hline WTEELEQYR & $131-139$ \\
\hline LTPVAQELK & $142-150$ \\
\hline LTPVAEEAR & $164-172$ \\
\hline NLAPYSDELR & $185-194$ \\
\hline GIPQASEYQAK & $208-218$ \\
\hline VMEQLSNLR & $219-227$ \\
\hline MTPLVQEFR & $230-238$ \\
\hline MTPLVQEFRER & $230-240$ \\
\hline LISFLDELQK & $252-261$ \\
\hline VAPTITLFPPSK & $4-15$ \\
\hline VAPTITLFPPSKEELNEATK & $4-23$ \\
\hline LEEQAKAK & $400-407$ \\
\hline SFWQHDEPQTPLDR & $20-33$ \\
\hline IRDMVDVYLETVK & $34-46$ \\
\hline DMVDVYLETVK & $36-46$ \\
\hline DAIAQFESSAVGK & $51-63$ \\
\hline LADNLDTLSAAAAK & $69-82$ \\
\hline IRPFLDQFSAK & $120-130$ \\
\hline WTEELEQYR & $131-139$ \\
\hline LTPVAQELK & $142-150$ \\
\hline LTPVAEEAR & $164-172$ \\
\hline NLAPYSDELR & $185-194$ \\
\hline GIPQASEYQAK & $208-218$ \\
\hline VMEQLSNLR & $219-227$ \\
\hline MTPLVQEFR & $230-238$ \\
\hline LISFLDELQK & $252-261$ \\
\hline VAPTITLFPPSK & $4-15$ \\
\hline VAPTITLFPPSKEELNEATK & $4-23$ \\
\hline VAPTITLFPPSK & $4-15$ \\
\hline ATLVCLINDFYPSPVTVDWVIDGSTR & $24-49$ \\
\hline KDPEGLFLQDNVVAQFTVDENGQMoxSATAK & $51-79$ \\
\hline YWGVASFLQK & $111-120$ \\
\hline GNDDHWVVDTDYDTYALHYSCR & $121-142$ \\
\hline NLAPYSDELR & $185-194$ \\
\hline GIPQASEYQAK & $208-218$ \\
\hline LISFLDELQK & $252-261$ \\
\hline KAADGTWQDFATGK & $58-71$ \\
\hline
\end{tabular}




\begin{tabular}{|c|c|}
\hline AADGTWQDFATGK & $59-71$ \\
\hline TTEFGEIHELTTEEQFVEGVYR & $72-93$ \\
\hline VEFDTSSYWK & $94-103$ \\
\hline GLGLSPFHEYADVVFTANDSGHR & $104-126$ \\
\hline HYTIAALLSPFSYSTTAVVSDPQE & $127-150$ \\
\hline YNDLKEETFK & $38-47$ \\
\hline AVAMITFAQYLQR & $48-60$ \\
\hline LVKDVVDLAQK & $69-79$ \\
\hline CVANEDAPECSKPLPSIILDEICQVEK & $80-106$ \\
\hline VSFLGHFIYSVAR & $160-172$ \\
\hline APFSEVSK & $254-261$ \\
\hline YIEDKEVCK & $337-345$ \\
\hline SFEAGHDAFMAEFVYEYSR & $346-364$ \\
\hline RHPEFSIQLIMR & $365-376$ \\
\hline MPQVPTDLLLETGK & $443-456$ \\
\hline MACSEGYLSIVIHDTCR & $474-490$ \\
\hline YVPPPFNPDMFSFDEK & $525-540$ \\
\hline TIADGFTAMVDK & $574-585$ \\
\hline QSDINTCFGEEGANLIVQSR & $589-608$ \\
\hline SVGNLADFEAELK & $4-16$ \\
\hline KVQEFSGANK & $85-94$ \\
\hline LADSHNFDEYMK & $11-22$ \\
\hline ALGVGFAMR & $23-31$ \\
\hline QVGNVTKPTVIISSEGDK & $32-49$ \\
\hline QVGNVTKPTVIISSEGDKVVIR & $32-53$ \\
\hline LGEEFDETTPDDR & $67-79$ \\
\hline SVVTLDGDK & 83-91 \\
\hline SVVTLDGDKLVHVQK & $83-97$ \\
\hline MVMTLTFGDVVAVR & $114-127$ \\
\hline LGEEFDETTPDDR & $67-79$ \\
\hline MVMTLTFGDVVAVR & $114-127$ \\
\hline LLGAPVPVDENDEGLQR & $30-46$ \\
\hline ALGVGFAMR & $23-31$ \\
\hline LLGAPVPVDENDEGLQR & $30-46$ \\
\hline ALQFAMAEYNR & $47-57$ \\
\hline RQLVSGIK & $75-82$ \\
\hline YILQVEIGR & 83-91 \\
\hline SSGDLQSCEFHDEPEMAK & $97-114$ \\
\hline YTTCTFVVYSIPWLNQIK & $115-132$ \\
\hline LLGAPVPVDENDEGLQR & $30-46$ \\
\hline ALQFAMAEYNR & $47-57$ \\
\hline QLVSGIKYILQVEIGR & $76-91$ \\
\hline YILQVEIGR & 83-91 \\
\hline AASHQEEFGAEALTR & $17-31$ \\
\hline GTEFTVNDLQGK & $120-131$ \\
\hline SAGWNIPIGTLLHR & $141-154$ \\
\hline GAIEWEGIESGSVEQAVAK & $155-173$ \\
\hline FFSASCVPGATIEQK & $174-188$ \\
\hline VEDIWSFLSK & $279-288$ \\
\hline
\end{tabular}




$\begin{array}{lc}\text { DQLTPSPR } & 353-360 \\ \text { FYTVISSLK } & 680-688 \\ \text { TCNPSDILQMCSFLEGK } & 689-705 \\ \text { HGLDNYR } & 33-39 \\ \text { GYSLGNWVCAAK } & 40-51 \\ \text { FESNFNTQATNR } & 52-63 \\ \text { NTDGSTDYGILQINSR } & 64-79 \\ \text { NLCNIPCSALLSSDITASVNCAK } & 92-114 \\ \text { CSTIIDAAFGSLK } & 287-299 \\ \text { SIPPACDK } & 1-8 \\ \text { SIPPACDKYSRLPGCPR } & 1-17 \\ \text { DYSPVCGTDGK } & 18-28 \\ \text { TYPNECVLCLSNSEENK } & 29-45 \\ \text { TYPNECVLCLSNSEENKNVQIYK } & 29-51 \\ \text { SVTGGMOXCSVYLK } & 998-1009 \\ \text { TYPNECVLCLSNSEENKNVQIYK } & 29-51 \\ \text { TDERPASYFAVAVAR } & 443-457 \\ & \\ \text { APSVDIGTCR } & 45-54 \\ \text { AGACPPTFTISGQCHGGLLNCCAK } & 41-64\end{array}$


Supplementary table 2A. Differentially expressed proteins in the seminal fluid of a Red Junglefowl (RJF) compared with White Leghorn (WL) male chicken was identified by 2D SDS-PAGE image comparison followed by mass spectrometry analysis.

\begin{tabular}{|l|l|l|l|l|}
\hline Spot no. & Proteins identified & \multirow{2}{*}{$\begin{array}{l}\text { Fold } \\
\text { change }\end{array}$} & \multicolumn{2}{l|}{ Average normalized volumes } \\
\cline { 5 - 5 } & & 2.0 & RJF & WL \\
\hline 108 & Serum albumin & 1.9 & $1.301 \mathrm{e}+007$ & $5.707 \mathrm{e}+006$ \\
\hline 109 & Serum albumin & 1.9 & $4.099 \mathrm{e}+007$ & $6.815 \mathrm{e}+007$ \\
\hline 122 & Ovotransferrin & 2.1 & $1.939 \mathrm{e}+007$ & $4.758 \mathrm{e}+007$ \\
\hline 123 & Ovotransferrin, Serum albumin & 2.4 & $2.460 \mathrm{e}+007$ & $5.790 \mathrm{e}+007$ \\
\hline 124 & Serum albumin & 3.2 & $1.331 \mathrm{e}+007$ & $4.223 \mathrm{e}+007$ \\
\hline 125 & Serum albumin & 4.3 & $2.379 \mathrm{e}+006$ & $1.033 \mathrm{e}+007$ \\
\hline 126 & Serum albumin & 1.9 & $1.277 \mathrm{e}+007$ & $2.452 \mathrm{e}+007$ \\
\hline 129 & Ovoinhibitor & 2.6 & $1.938 \mathrm{e}+006$ & $5.076 \mathrm{e}+006$ \\
\hline 131 & Creatinine kinase B type & 5.0 & $2.118 \mathrm{e}+006$ & $1.060 \mathrm{e}+007$ \\
\hline 132 & A-enolase, $\beta$-enolase & 1.7 & $2.752 \mathrm{e}+007$ & $1.604 \mathrm{e}+007$ \\
\hline 139 & Malate dehydrogenase & 5.3 & $7.507 \mathrm{e}+006$ & $3.941 \mathrm{e}+007$ \\
\hline 140 & L-lactate dehydrogenase B chain & 5.3 & $5.260 \mathrm{e}+007$ & $1.731 \mathrm{e}+007$ \\
\hline 141 & $\begin{array}{l}\text { Glyceraldehyde-3-phosphate } \\
\text { dehydrogenase, }\end{array}$ & 3.0 & & \\
& $\begin{array}{l}\text { Astacin-like } \\
\text { metalloendopeptidase }\end{array}$ & & & \\
\hline 145 & Triosephosphate isomerase & 9.1 & $1.637 \mathrm{e}+006$ & $1.493 \mathrm{e}+007$ \\
\hline 146 & Retinol-binding protein 4 & 3.4 & $8.781 \mathrm{e}+006$ & $2.987 \mathrm{e}+007$ \\
\hline 147 & Apolipoprotein A-I & 3.8 & $1.032 \mathrm{e}+006$ & $3.972 \mathrm{e}+006$ \\
\hline 152 & Cystatin & 2.7 & $3.704 \mathrm{e}+007$ & $1.397 \mathrm{e}+007$ \\
\hline 157 & Gallinacin-9 & 1.9 & $2.800 \mathrm{e}+008$ & $1.508 \mathrm{e}+008$ \\
\hline
\end{tabular}


Supplementary table 2B. Differentially expressed proteins in the seminal fluid of Red Junglefowl (RJF) compared with an Advanced Intercross Line (AIL) male chicken was identified by 2D SDS-PAGE image comparison followed by mass spectrometry analysis.

\begin{tabular}{|l|l|l|l|l|}
\hline \multirow{2}{*}{$\begin{array}{l}\text { Spot } \\
\text { no. }\end{array}$} & Proteins identified & \multirow{2}{*}{ Fold change } & \multicolumn{2}{|l|}{ Average normalized volumes } \\
\cline { 4 - 5 } & & & RJF & AlL \\
\hline 162 & Protein NEL & 2.3 & $2.15 \mathrm{e}+006$ & $9.42 \mathrm{e}+005$ \\
\hline 172 & Tubulin beta- 1 chain & 2.0 & $7.50 \mathrm{e}+005$ & $1.50 \mathrm{e}+006$ \\
\hline 192 & Ovotransferrin & 4.0 & $1.73 \mathrm{e}+005$ & $6.95 \mathrm{e}+005$ \\
\hline 193 & Ovotransferrin & 3.6 & $7.12 \mathrm{e}+005$ & $2.56 \mathrm{e}+006$ \\
\hline 214 & Serum albumin & 3.2 & $6.99 \mathrm{e}+005$ & $2.26 \mathrm{e}+006$ \\
\hline 230 & Phosphoglycerate mutase 1 & 2.1 & $4.19 \mathrm{e}+005$ & $8.84 \mathrm{e}+005$ \\
\hline 238 & Transthyretin & 2.6 & $1.99 \mathrm{e}+006$ & $7.68 \mathrm{e}+005$ \\
\hline 243 & Ig lambda chain C region & 2.0 & $3.49 \mathrm{e}+006$ & $1.71 \mathrm{e}+006$ \\
\hline & & 2.9 & $8.87 \mathrm{e}+005$ & $3.11 \mathrm{e}+005$ \\
\hline 18 & Ovotransferrin & 2.6 & $7.10 \mathrm{e}+005$ & $2.75 \mathrm{e}+005$ \\
& & 3.4 & $2.52 \mathrm{e}+006$ & $7.42 \mathrm{e}+005$ \\
\hline 82 & Argininosuccinate synthase & 3.1 & $1.22 \mathrm{e}+006$ & $4.00 \mathrm{e}+005$ \\
\hline 85 & Transthyretin & 2.9 & $1.59 \mathrm{e}+006$ & $5.53 \mathrm{e}+005$ \\
\hline 89 & Serum albumin & 2.1 & $5.87 \mathrm{e}+005$ & $2.78 \mathrm{e}+005$ \\
\hline
\end{tabular}

